\title{
PROCESSING OF SIMULATED AND EXPERIMENTAL IMAGES OF CLOSELY SPACED BINARY STARS USING SPECKLE INTERFEROMETRY
}

\author{
A Thesis \\ presented to \\ the Faculty of California Polytechnic State University, \\ San Luis Obispo
}

\author{
In Partial Fulfillment \\ of the Requirements for the Degree \\ Master of Science in Electrical Engineering
}

by

Niels Christian Smidth

June 2016 
(C)2016

Niels Christian Smidth

\section{ALL RIGHTS RESERVED}


COMMITTEE MEMBERSHIP

TITLE: Processing of Simulated and Experimental Images of Closely Spaced Binary Stars Using Speckle Interferometry

AUTHOR: Niels Christian Smidth

DATE SUBMITTED: June 2016

COMMITTEE CHAIR: John Ridgely, Ph.D.

Professor of Mechanical Engineering

COMMITTEE MEMBER: Russell Genet, Ph.D.

Research Scholar in Residence

COMMITTEE MEMBER: Jane Zhang, Ph.D.

Professor of Electrical Engineering 


\section{ABSTRACT \\ Processing of Simulated and Experimental Images of Closely \\ Spaced Binary Stars Using Speckle Interferometry \\ Niels Christian Smidth}

Theory and methods of processing speckle interferometry data from close visual binary stars are presented and implemented. The effects of the optical systems used for observing close visual binary stars are explained and simulated from both the geometrical and physical optical viewpoints. The atmospheric phase distortion and shot noise responsible for the observed speckle patterns are simulated. The deconvolution technique originally presented by Labeyrie is implemented to extract astrometric data from close visual binary stars. This method is applied to both simulated and experimental data from Kitt Peak National Observatory as validation. Parts of the deconvolution process are optimized to allow for near real time calculations in an automated observatory. 


\section{ACKNOWLEDGMENTS}

I would like to thank my advisor, John Ridgely, for all the time he has spent on this project while on sabbatical. In addition, I have greatly appreciated Russell Genet's countless hours spent getting me up to speed as well as for his unrelenting passion to the advancement of astronomy and getting students involved with research. A big thanks to David Rowe as well, who was instrumental in helping my understanding of some of the most difficult to understand concepts of this project. Lastly, I have to express my gratitude to my family, who have have been incredibly helpful in both technical and moral support. 
LIST OF FIGURES . . . . . . . . . . . . . . . . . . . . . . . viii

\section{CHAPTER}

1 INTRODUCTION . . . . . . . . . . . . . . . . . . . . 1

1.1 Objective $\ldots \ldots \ldots \ldots \ldots \ldots \ldots \ldots \ldots \ldots \ldots \ldots \ldots$

1.2 Motivation ....................... 1

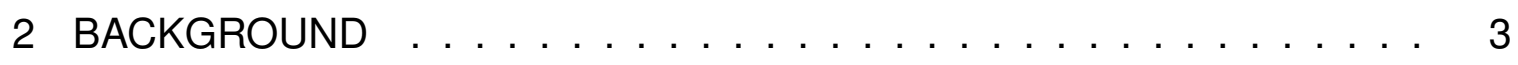

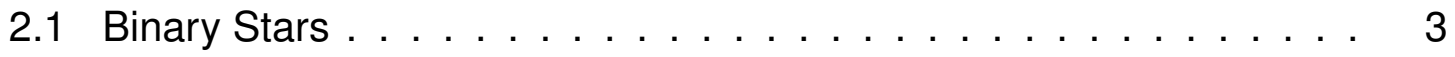

2.2 Telescope Theory. . . . . . . . . . . . . . . . . . . . 4

2.3 Optical Transfer Function and Fourier Optics . . . . . . . . . . . 10

2.4 Telescope Seeing Limit . . . . . . . . . . . . . . . . . . . . . . 14

2.5 Atmospheric Distortion . . . . . . . . . . . . . . . . 18

3 LABEYRIE DECONVOLUTION . . . . . . . . . . . . . . . . . . 26

3.1 Labeyrie's Findings . . . . . . . . . . . . . . . . 26

3.2 Theory . . . . . . . . . . . . . . 27

3.3 Implementation . . . . . . . . . . . . . . . . 44

4 IMPLEMENTATION IN AUTOMATED OBSERVATORY . . . . . . . . . . 46

4.1 Optimization . . . . . . . . . . . . . . 46

4.2 Implementation $\ldots \ldots \ldots \ldots \ldots \ldots \ldots \ldots \ldots$

5 FUTURE WORK $\ldots \ldots \ldots \ldots \ldots \ldots$ 


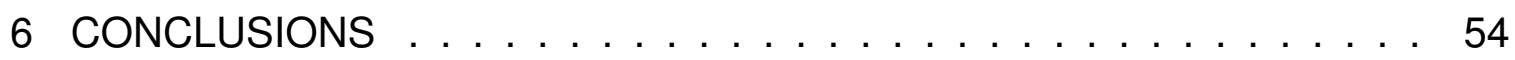

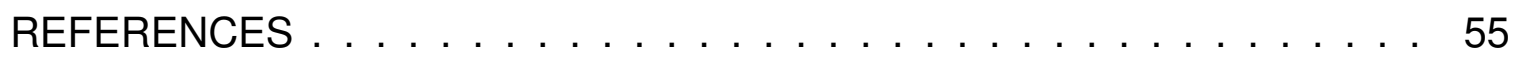

\section{APPENDICES}

A Prime Focus Geometric Optics . . . . . . . . . . . . . . . 59

B Prime Focus Diffraction Limit Simulation . . . . . . . . . . . . . . . 62

C Atmospheric Distortion Simulation $\ldots \ldots$. . . . . . . . 67

D Atmospheric Distortion Simulation Class . . . . . . . . . . . . . 71

E Labeyrie Deconvolution Simulations $\ldots \ldots \ldots$. . . . . . 76

F Python Classes for Speckle Data Processing . . . . . . . . . . . . . . . 86

G Processing FITS Data using classes_labeyrie.py $\ldots \ldots \ldots . \ldots . . . .99$

H Benchmarking PSD Computation Methods . . . . . . . . . . . . . . . . 104 


\section{LIST OF FIGURES}

Figure $\quad$ Page

2.1 The orbits of a binary star pair $[1] \ldots \ldots \ldots$

2.2 Ray trace diagram of a prime focus telescope $\ldots \ldots \ldots \ldots$

2.3 Ray trace diagram sign convention $\ldots \ldots \ldots \ldots$

2.4 Model of a celestial object as point source $\ldots \ldots \ldots \ldots$

2.5 Kitt Peak $2.1 \mathrm{~m}$ mirror simulation . . . . . . . . . . . . . . 9

2.6 Behavior of light through an aperture $[2] \ldots \ldots \ldots \ldots \ldots$

2.7 Relationship between spatial and spatial frequency domains . . . . . 12

2.8 Using the Fraunhofer diffraction to calculate PSF of pupil . . . . . . 14

2.9 KPNO 2.1m telescope PSF (logarithmic intensity) . . . . . . . . . 16

2.10 Diffraction limited binary star simulation $\ldots \ldots \ldots \ldots$

2.11 Center row of aperture diffraction image $\ldots \ldots \ldots \ldots$

2.12 Minimum spacing as defined by Rayleigh Criterion [3] $\ldots \ldots \ldots$

2.13 Creation of atmospheric turbulence $[4] \ldots \ldots \ldots \ldots$

2.14 Atmospheric distortion's effect on telescope images [5] . . . . . . . 20

2.15 Phase and magnitude of complex pupil image . . . . . . . . 21

2.16 Simulated PSF through atmospheric distortion $\ldots \ldots . . \ldots 21$

2.17 Adding shot noise to simulated image $\ldots \ldots \ldots . \ldots 23$

2.18 Adding Gaussian read noise to simulated image $\ldots \ldots \ldots$

3.1 Short vs long exposure times through atmospheric distortion $\ldots 26$

3.2 Average PSD and autocorrelation of binary star images $\ldots \ldots .28$ 
3.3 Recovered binary star object PSD/autocorrelation . . . . . . . . . 32

3.4 Sources of signal energy in noiseless PSD $\ldots \ldots \ldots \ldots$

3.5 Domination of high frequency noise in inverse filtering $\ldots \ldots \ldots$

3.6 Wiener filter inverse filter region . . . . . . . . . . . . . . 39

3.7 Results from simplified Wiener filter deconvolution . . . . . . . . 39

3.8 Binary and Reference star data from KPNO $\ldots \ldots \ldots \ldots$

3.9 Applying simplified Wiener filter to KPNO data . . . . . . . . . . . 41

3.10 Applying second Wiener filter to KPNO data . . . . . . . . . . 42

3.11 Comparison of inverse filtering regions of Wiener filters . . . . . . 43

3.12 Deconvolution KPNO data with LPF/Inverse Filtering . . . . . . . . 44

4.1 Workflow with PS3 Software . . . . . . . . . . . . . . 46

4.2 Comparison of PSD Calculation Techniques . . . . . . . . . . 47

4.3 Present workflow for binary star observations . . . . . . . . . . 51

4.4 Proposed workflow for binary star observations . . . . . . . . . 52 


\section{INTRODUCTION}

\subsection{Objective}

Observing binary stars using speckle interferometry requires software tools to extract the astrometric measurements. This project is concerned with presenting research on the theory behind speckle interferometry as well as creating well documented speckle interferometry software tools in a modern, cross platform language that allow for continual development by further students.

\subsection{Motivation}

The theory behind the observation of close visual binary stars with speckle interferometry is a multi-disciplinary task, spanning the fields of physics, Fourier optics, image processing, and computation. Creating simulations of the phenomena behind speckle interferometry allows students more knowledgeable in a single field of the process to understand the entire process and work with experts of the other fields.

Observing closely spaced binary stars requires a large telescope, which provides a high enough angular resolution to see the two distinct targets. Getting observing time on a 3-meter or greater telescope required to make these observations is quite difficult for academic groups today. To allow for academic groups to easily perform double star observations using speckle interferometry, the construction of a suitable automated telescope has been proposed by Russell Genet et al. [6]. This proposed 4-meter class telescope has been estimated to cost nearly a 
hundredth of what a conventional telescope of this class might cost. Part of this project involves creating a software tool chain allowing students from different academic institutions to make observations and get processed data remotely. Like the design of the telescope, this software should be modular and well documented so that it may be modified by students and instructors as the scope of the project changes over time. 


\section{BACKGROUND}

\subsection{Binary Stars}

It is estimated that up to two thirds of solar stars nearby have stellar companions [7]. Many of these systems may be described as binary stars, a pair of gravitationally bound stars. Each star has its own elliptical orbit around the common focus known as the barycenter, the pair's common center of mass, as seen in Figure 2.1. Binary stars are of great interest to astronomers as their visible interaction with each other gives the opportunity to calculate the mass of the total system and the individual stars. The mass of a star gives important insight into the lifecycle of the star, making it valuable in research on the evolution of the universe and modeling stellar behavior.

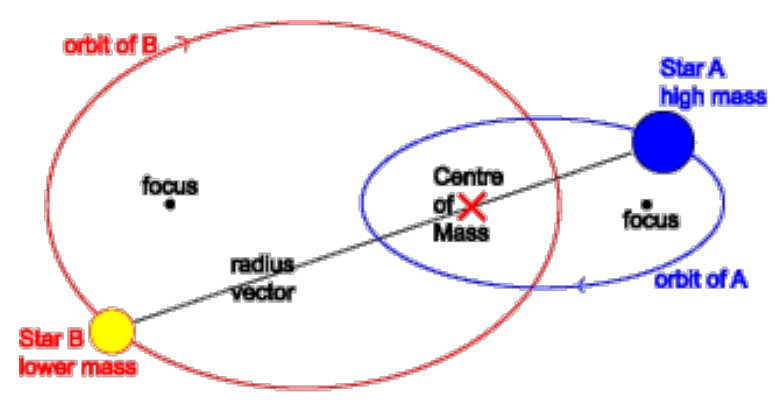

\section{Orbits of Stars in a Binary System}

Figure 2.1: The orbits of a binary star pair [1]

Many of these binary star pairs are either too far away from Earth or too closely spaced from each other to be able to visually resolved. These may be studied using techniques such as spectroscopy to view the Doppler shifts spectrum from radial velocity changes. This thesis is concerned with visual binary stars, which are spaced far apart enough to be visually resolved with a telescope. Using telescopes, 
the separation and position angle of the orbit may be observed over time, allowing the use of Kepler's laws of planetary motion to calculate masses.

\subsection{Telescope Theory}

When viewing a very distant object with the unaided eye, its projection onto the retina is very small compared to the area of the retina. This small projection covers very few rods/cones on the retina, which may be compared to the pixels on a digital camera's CCD. Because only a few pixels receive the projection, we are unable to make out significant detail on the distant object. For example, a binary star appears as a bright point in the sky to the naked eye, rather than two separate visible spheres. Due to the unaided eye's inability to study such distant objects, we use a telescope to collect and concentrate the light from distant objects onto an imaging sensor, which may be the eye or a CCD sensor. The ability of a telescope with an image sensor to form a usable image of a binary star may be explained with concepts from geometrical optics.

The telescopes commonly used in viewing visual binary stars are variations on a basic reflecting telescope design. A reflecting telescope uses one or several shaped mirrors to focus the incoming light from the target into an image. The simplest single mirror reflecting telescope is the prime focus telescope, depicted in Figure 2.2. A prime focus telescope uses a single parabolic mirror to focus incoming parallel light rays into a single focal point [3]. Important dimensions of this mirror are its focal length and diameter, also depicted in Figure 2.2. 


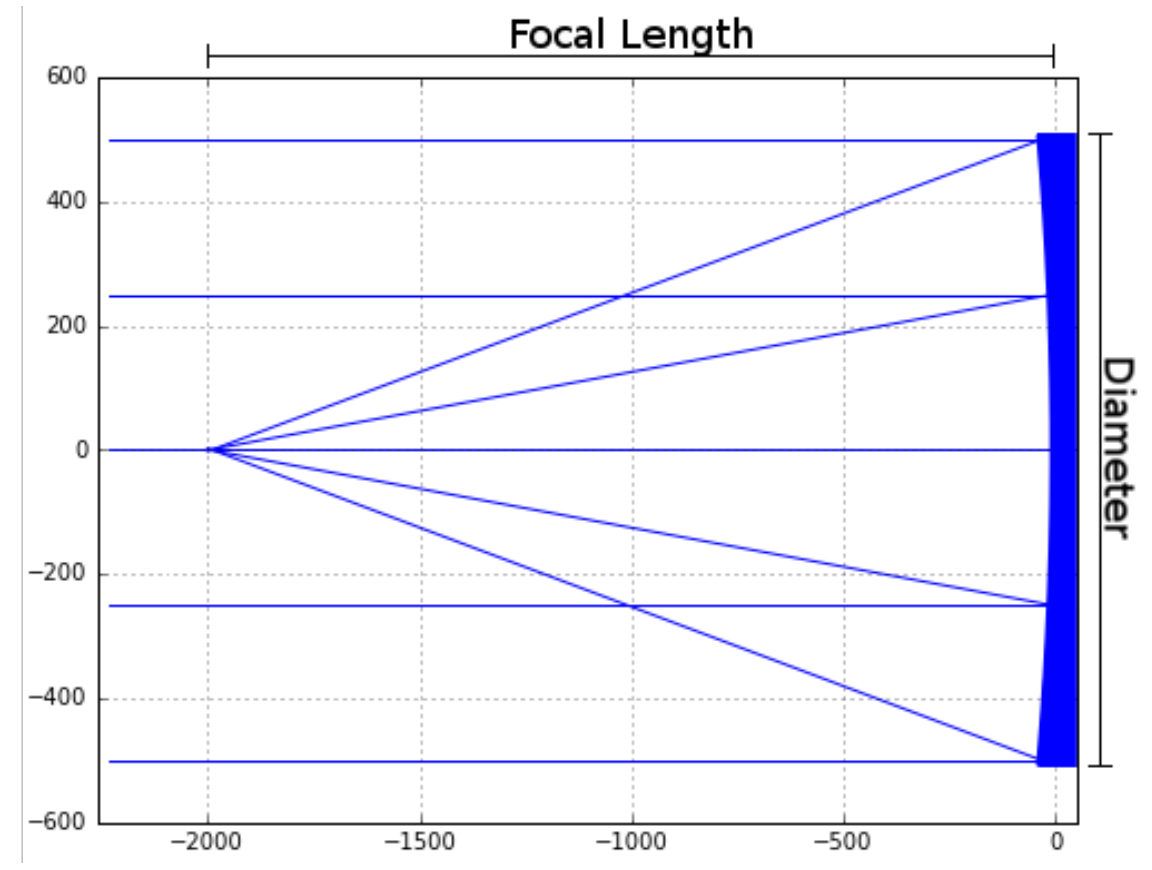

Figure 2.2: Ray trace diagram of a prime focus telescope

For this discussion of basic functioning of a telescope, we can consider these primary mirrors to be in the size range of 0.5 meters, the size of a serious amateur telescope, all the way up to $10 \mathrm{~m}$, the size of one of the two Keck telescopes on Mauna Kea. These telescope mirrors are many magnitudes larger than the wavelength of visible light, in the band of $390 \mathrm{~nm}-700 \mathrm{~nm}$. This means that the wave properties of light such as diffraction and interference occur on a scale considerably smaller than the mirrors, so we may approximate light as rays traveling in a straight line interacting with geometrical optics [8]. For example: in Figure 2.2, the parabolic mirror's ability to focus incoming parallel light rays is shown using the geometrical optics approximation.

In order to simplify the analysis of these geometrical optics, some approximations may be made. If the incident and reflected light rays (see Figure 2.3 ) are 
at a small angle with respect to the optical axis, the paraxial approximation can be made [9]. The paraxial approximation allows the following simplifications to be made:

$$
\sin (\alpha)=\alpha \quad \cos (\alpha)=1 \quad \tan (\alpha)=\alpha
$$

These simplifications make calculating reflected angles much easier, as well as allowing the use of ray transfer matrices for calculating incident and reflected angles of light rays.

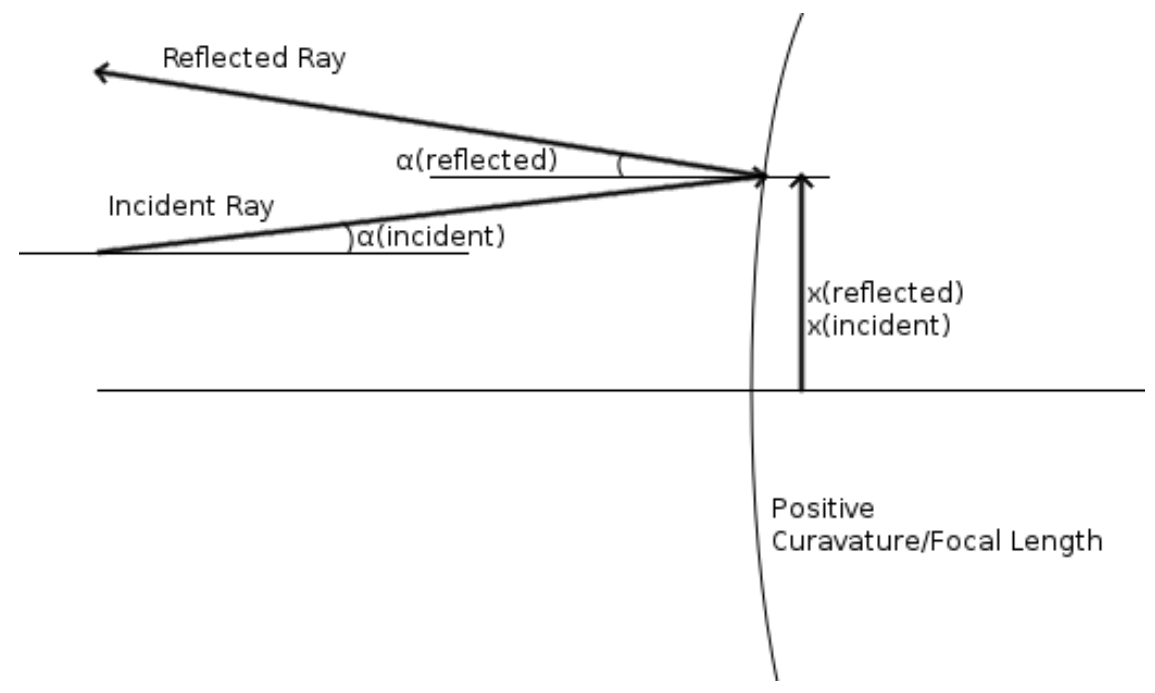

Figure 2.3: Ray trace diagram sign convention

The ray transfer matrix method is used to calculate the height and angle of reflected/refracted rays given the shape of the interface as well as the height and angle of the incident rays. The general form of the matrix is:

$$
\left[\begin{array}{c}
\alpha_{\text {reflected }} \\
x_{\text {reflected }}
\end{array}\right]=\left[\begin{array}{ll}
A & B \\
C & D
\end{array}\right]\left[\begin{array}{l}
\alpha_{\text {incident }} \\
x_{\text {incident }}
\end{array}\right]
$$

The values in the $A B C D$ matrix are related to the interface the light rays interact with. For a parabolic concave mirror, the ray transfer matrix is defined as [10]: 


$$
\left[\begin{array}{c}
\alpha_{\text {reflected }} \\
x_{\text {reflected }}
\end{array}\right]=\left[\begin{array}{cc}
1 & -\frac{1}{f} \\
0 & 1
\end{array}\right]\left[\begin{array}{l}
\alpha_{\text {incident }} \\
x_{\text {incident }}
\end{array}\right]
$$

This ray transfer matrix is used in a simulation (See Appendix 6) of the Kitt Peak National Observatory $2.1 \mathrm{~m}$ telescope to show the parabolic mirror's ability to focus parallel light rays incident at different angles. The results of this simulation are shown in 2.5. When mirrors with a focal length several times greater than mirror diameter are used, reflected angles are small and the paraxial approximation remains valid. In reality, comatic aberration, which causes distortion of the image, increases with increasing incident ray angle [4]. This may be ignored for this discussion as the telescope configurations used in binary star observations typically have a small field of view, and thus small light ray angles, to give high detail when viewing objects with small features.

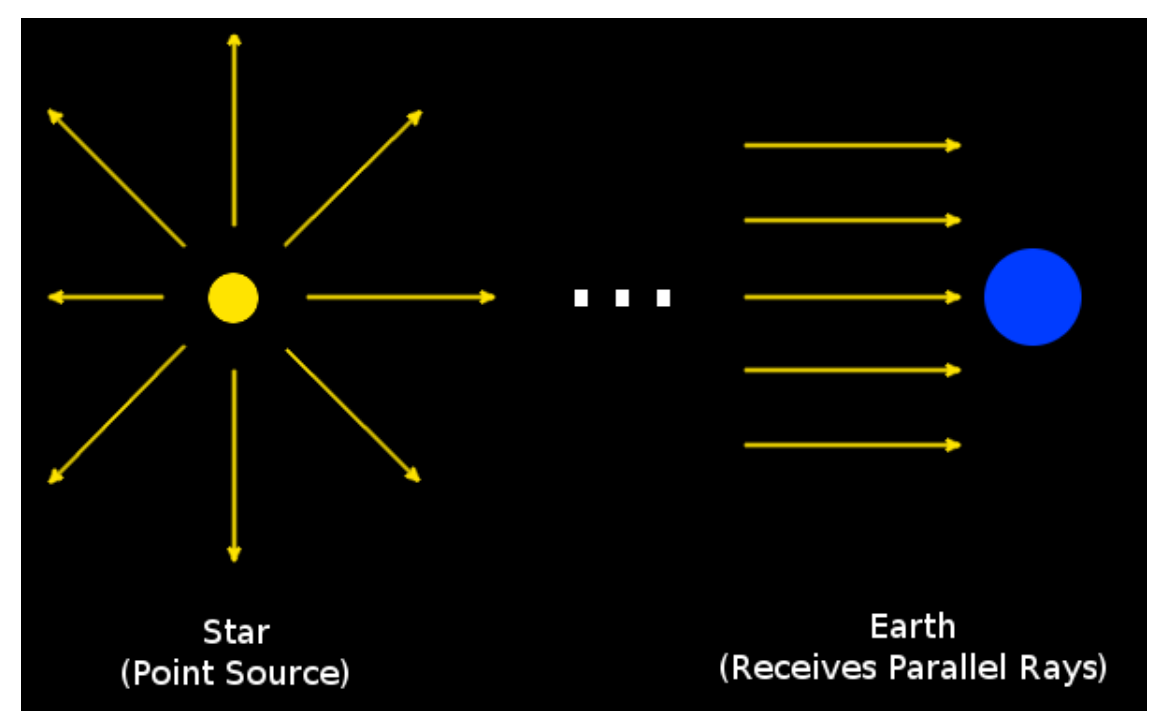

Figure 2.4: Model of a celestial object as point source

Distant objects like stars may be modeled as point sources of light, giving off light rays evenly in every direction. Because these stars are at an effectively infinite 
distance, these light rays become effectively parallel by the time they reach the earth, as shown in Figure 2.4 [4]. This behavior may be applied to all distant celestial objects in our field of view, each emitting light that becomes a parallel set of rays by the time they reach the earth.

The difference between the parallel rays from these different sources is their incident angles. As shown in Figure 2.5, each parallel bundle of light rays from a specific angle is focused to a different unique point. The image formed on an image sensor is the superposition of all the different angled parallel light ray bundles, each focused down to a point on the image sensor.

This knowledge allows for calculation of the image scale of a telescope, which gives the relation between subtended angles in the sky and distances on the image sensor. With this knowledge, the field of view and pixel scales may be calculated as well [4]. These quantities allow for converting between units in the image plane and units in the sky.

$$
\text { image scale }[\operatorname{arcsec} / m]=\frac{206265[\operatorname{arcsec}]}{\text { focal length }[m]}
$$

field of view $[\operatorname{arcsec}]=($ image scale $[\operatorname{arcsec} / m]) *($ image sensor width $[m])$ pixel scale $[\operatorname{arcsec} /$ pixel $]=($ image scale $[\operatorname{arcsec} / m]) *($ pixel width $[m])$

The ability of a telescope to form the image of a celestial scene was explained using geometrical optics. With this understanding, a binary star would reduce down to two perfect points of light on the image sensor, giving the viewer the ability to precisely measure the angle and distance between the stars. The Andor Luca 

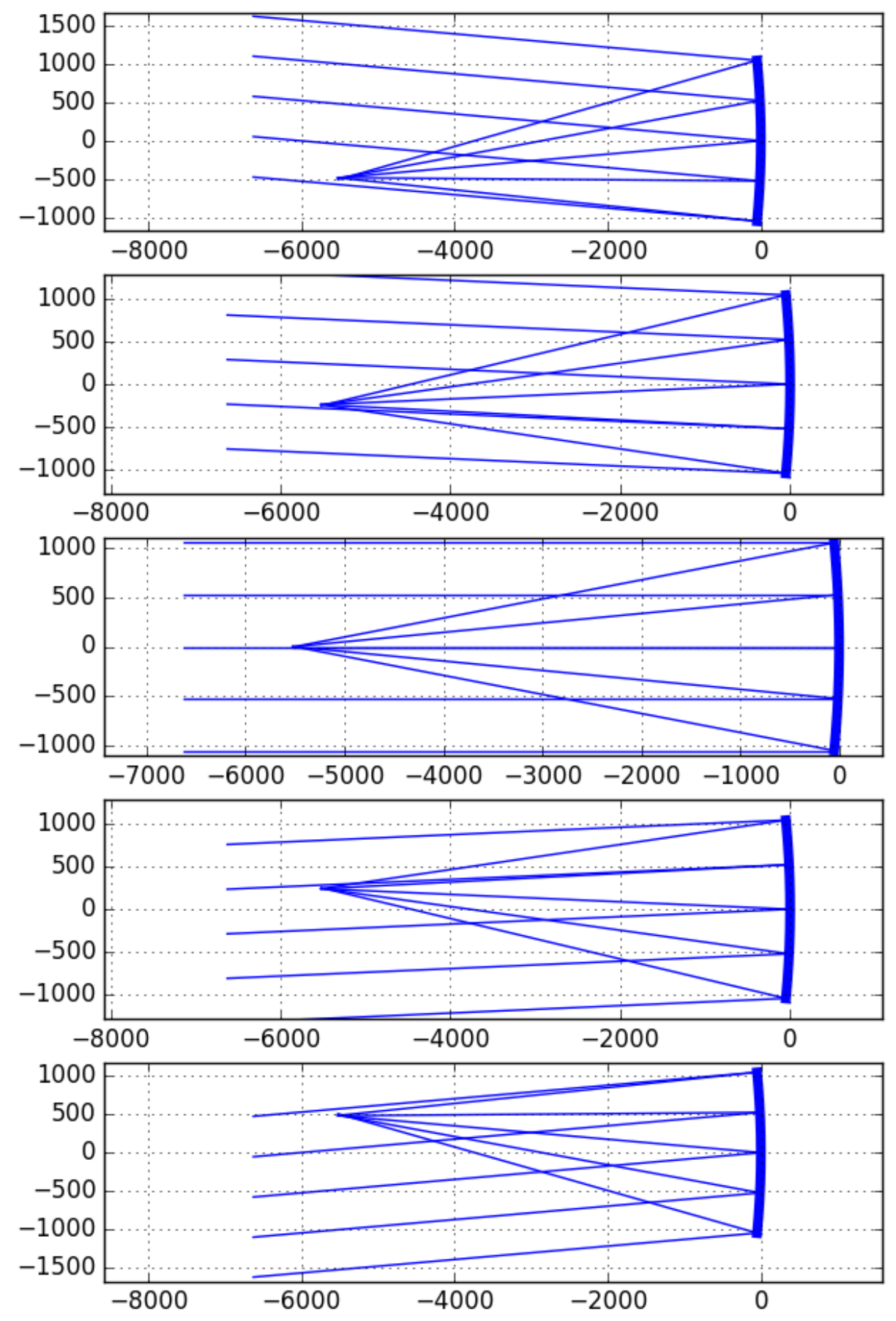

Figure 2.5: Kitt Peak 2.1m mirror simulation

R EMCCD camera, used by Russell Genet et al in their binary star observations at Kitt Peak Observatory [11], has a pixel size of only $8 \mu m \times 8 \mu m$ [12]. Images captured with this much detail will reveal the more complex interference effects of 
light, making the geometrical optics model not accurate enough. In order to fully model the behavior of the telescope and image sensor combination, techniques involving the wave nature of light must be used.

\subsection{Optical Transfer Function and Fourier Optics}

When considering light in geometrical optics, rays are used to show the transmission of light through interfaces such as lenses and apertures. Each ray follows straight lines, changing direction when transmitting through different materials (refraction) or encountering a reflective surface (reflection). This knowledge allowed for the use of ray trace matrices in showing the behavior of an optical system. When rays pass through an aperture, those outside the diameter of the aperture are stopped and absorbed, while those within the diameter pass through unaffected. When considering the electromagnetic wave behavior of light, an interface such as a lens causes change in the wave's phase at each spatial coordinate, resulting in the changing of the wavefront's direction. An aperture now causes a diffraction pattern to be visible in the light intensity field produced on a screen after the aperture. The difference between wave and ray consideration of light with an aperture is shown in Figure 2.6. So in wave optics, an interface is modeled by considering the changes that incident light undergoes in magnitude and phase at each spatial point.

When considering the wave nature of light, optical systems may be analyzed with similar techniques as are employed when modeling responses of circuits or mass-spring-damper systems to an input [3]. The signals involved in optical sys- 


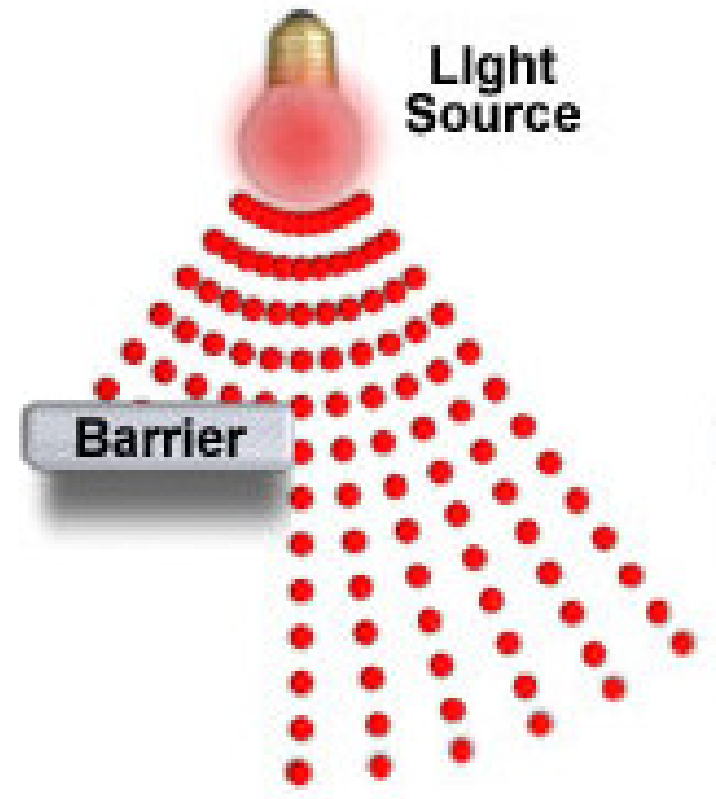

Particles

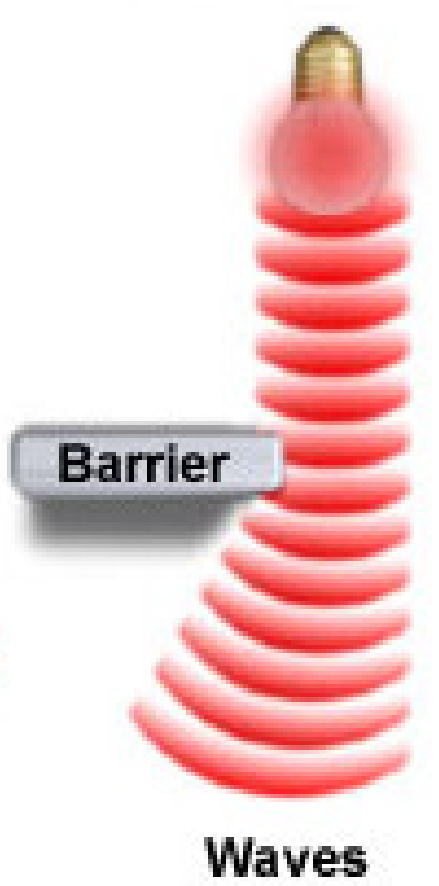

Waves

Figure 2.6: Behavior of light through an aperture [2]

tems are two-dimensional spatial signals, rather than the one-dimensional temporal signals commonly used when modeling systems. An optical system may be modeled by its Point Spread Function (PSF), the intensity pattern produced in response to the input of a single point of light. The optical system may also be modeled in the spatial frequency domain by its Optical Transfer Function (OTF), which forms a Fourier pair with the PSF. The response of an optical system to an input may be calculated as shown in 2.7 .

It is important to note that these responses are only valid for a source that is spatially and temporally coherent. Coherence refers to the strength of correlation between signals at different times or locations and is related to the ability to produce interference effects [13] [14]. A temporally coherent source has little random fluctuation in wavefront spacing over time. This means that at a single observing 


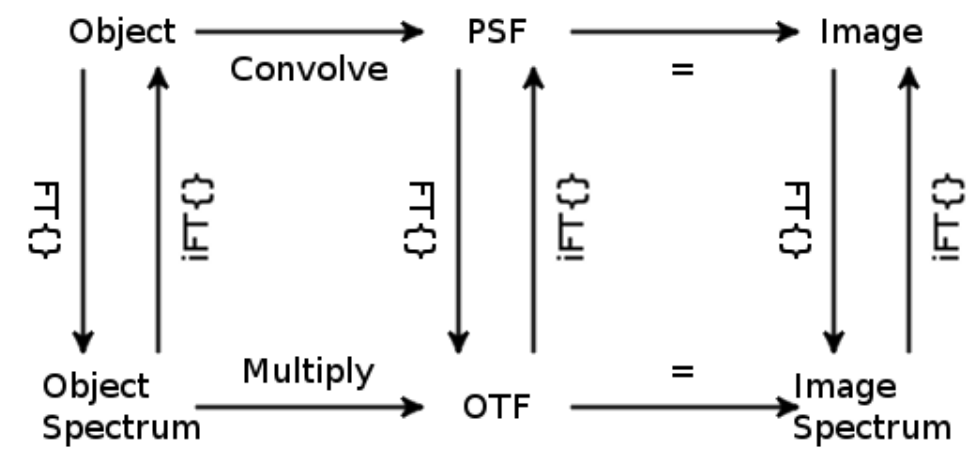

Figure 2.7: Relationship between spatial and spatial frequency domains

point but different times, observed light over a time interval will exhibit high levels of correlation. A spatially coherent source has little variation in wavefront spacing at different points. This means that at different observing points but at the same time, observed light over a time interval will exhibit high levels of correlation. An example of a near ideal source (both spatially and temporally coherent) is a laser, which is very near an ideal point source (making it spatially coherent) and produces nearly monochromatic light (making it temporally coherent). The sun is neither spatially nor temporally coherent, as it is considerably larger than a point source, even to the naked eye, and is a very wideband source. The binary stars under observation are considerably farther away than the sun, making them effectively point sources (and therefore spatially coherent). Using a narrowband filter in the observing telescope makes the binary star appear as temporally coherent as well. Since filtered binary stars may be considered as temporally and spatially coherent sources, this OTF analysis may be applied to their observation. For spatially incoherent sources, this OTF analysis may no longer be applied. The lack of coherence prevents interfer- 
ence, the output signal is defined as the superposition of intensity images (rather than the superposition of phasors for coherent imaging) [14].

The prime focus telescope modeled previously using geometrical optics may also be fully modeled using wave optics, but this is a large task and most of the important behavior of this telescope due to the wave nature of light may be shown using some approximations. The dominant diffraction effect in a telescope is due to the finite size of optical components. At the far field distance, where the optical path length differences from points at dimensional extremes of an optical component is significantly less than the wavelength, the Fraunhofer diffraction pattern is generated [15]. The distance at which this diffraction pattern occurs can be estimated in several ways, one common method is the "antenna designer's formula"[16]:

$$
z>\frac{2 D^{2}}{\lambda}
$$

where $z$ is the separation between the plane of the optical component and the beginning of the far field, $D$ is the largest diameter of the optical component, and $\lambda$ is the wavelength of the light.

The primary parabolic lens or mirror of a telescope may be modeled as a finite sized aperture (with $D=$ diameter of primary lens) over a larger lens, so incoming light outside of $D$ is not transmitted. As an example, for $0.6 \mu \mathrm{m}$ red light with a $2.1 \mathrm{~m}$ aperture diameter, the far field occurs at $14.7 * 10^{6}$ meters away from the aperture. Distances of this amplitude are considerably larger than the lengths of most telescopes. However, the parabolic lens focuses this light, which would normally show the Fraunhofer diffraction at a very large distance, at its focal length. Thus in a 
simple telescope system, the Fraunhofer diffraction pattern of the primary lens is shown on the image sensor.

Under these conditions, the complex diffraction pattern at the focal plane is calculated as the Fourier transform of the complex valued pupil image. In reality, there are other phase terms and higher order terms in the diffraction pattern, but for the small field of view found in the telescopes used in speckle interferometry, these may be ignored. This may be used to calculate the PSF of different components of the optical system as shown in Figure 2.8. These techniques will be used to analyze the response of a simple Prime Focus telescope as well as the effects of atmospheric distortion.

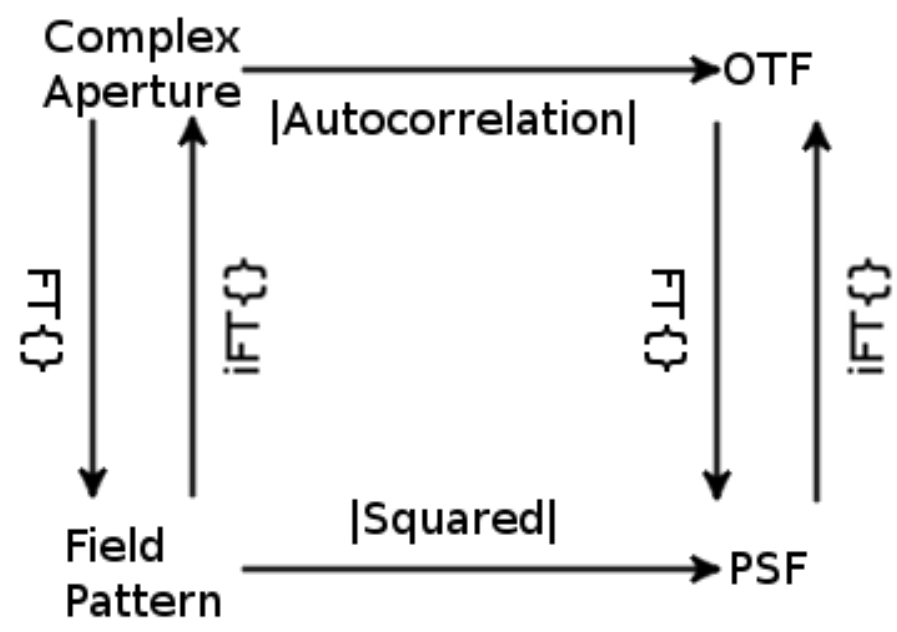

Figure 2.8: Using the Fraunhofer diffraction to calculate PSF of pupil

\subsection{Telescope Seeing Limit}

Using geometrical optics analysis, a prime focus telescope will produce a perfect image of a star on the image sensor, which will appear as a tiny point/circle. Taking 
into account the wave model of light, the more accurate image produced may be calculated.

The OTF technique illustrated in Figure 2.7 is utilized to simulate the Kitt Peak National Observatory's (KPNO) 2.1m telescope, as seen by the Andor Luca-R camera (Appendix B). This simulation uses a Fast Fourier Transform rather than a continuous Fourier Transform to calculate the PSF of the pupil function. The PSF of this telescope's main mirror (as seen in an Andora Luca-R camera) is shown in 2.9. This PSF shape, resulting from the diffraction pattern of a circular aperture (with magnitude $=1$ inside the aperture, magnitude $=0$ outside the aperture), is known as an Airy Disk. This Airy disk is shown in a logarithmic intensity scale to accentuate the fringes that surround the central peak. A simulated binary star and the simulated output image is shown in Figure 2.10. The output image is explained as two Airy Disks, each superposed at the location of the point source input.

The effects of diffraction on the output image of a binary star can be more easily viewed in one dimension in Figure 2.11. In one dimension, the Airy disk bears a strong resemblance to the sinc function. The first null of this function occurs in the image sensor plane at a distance from center of

$$
\text { radius }=1.22 * \lambda * \frac{\text { focal length }}{\text { lens diameter }}
$$

The Rayleigh Criterion states that this radius is the minimum distance between two distinguishable point objects in an optical system. This distance is shown in 2.12. As the distance between two point objects gets smaller than this distance, the central peak of the two Airy disks begin to smudge together, preventing iden- 


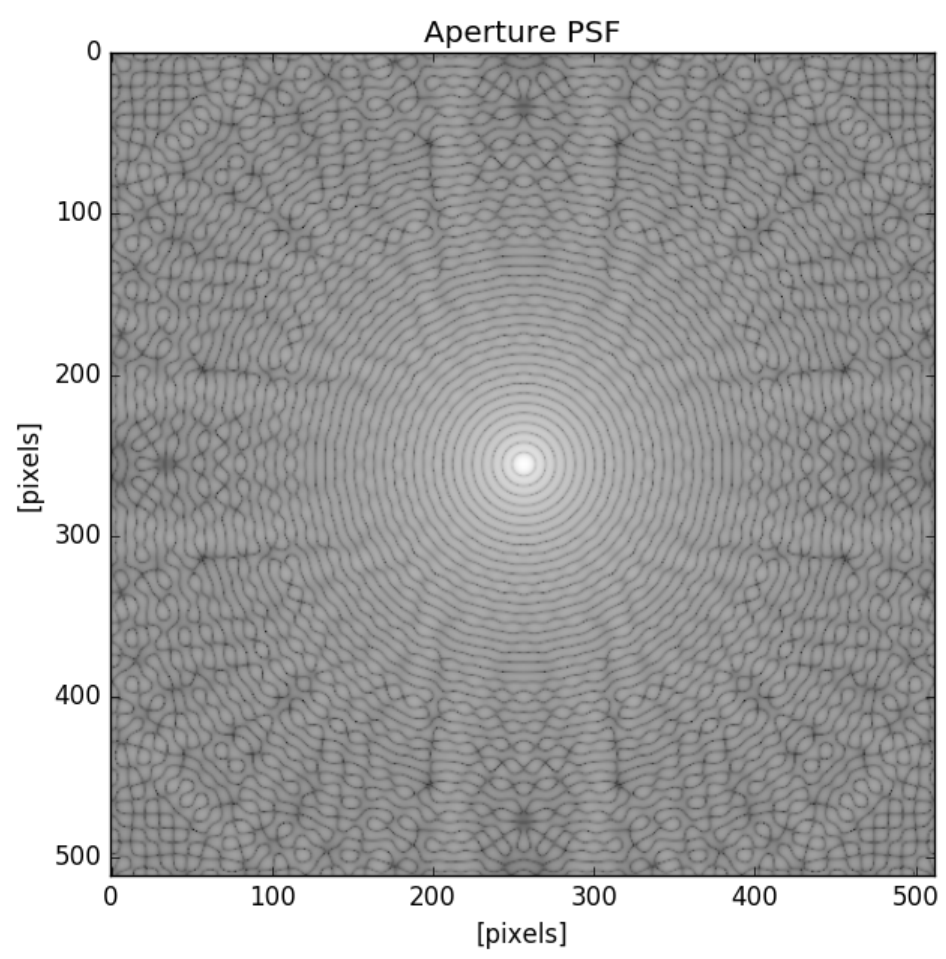

Figure 2.9: KPNO 2.1m telescope PSF (logarithmic intensity)
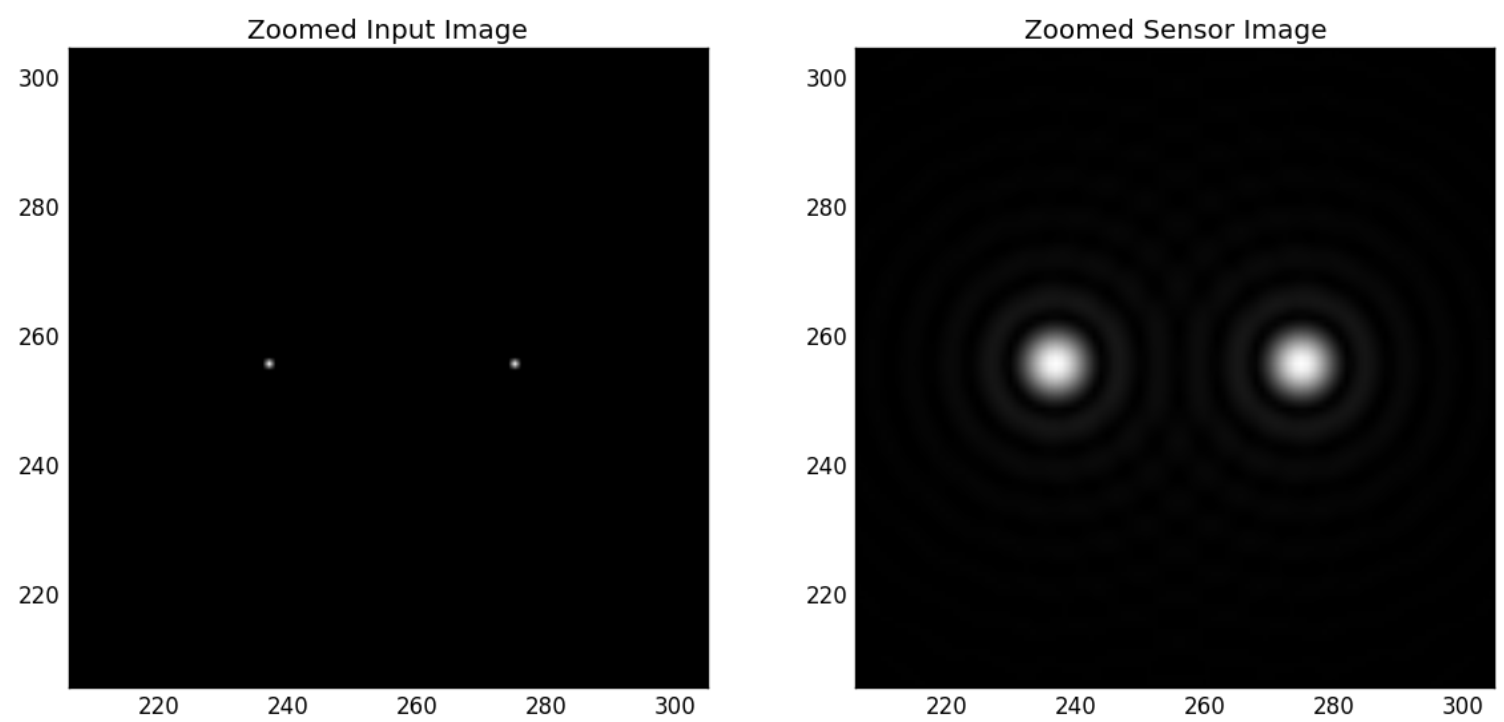

Figure 2.10: Diffraction limited binary star simulation

tification of each individual peak's location. In order to resolve objects with fine 

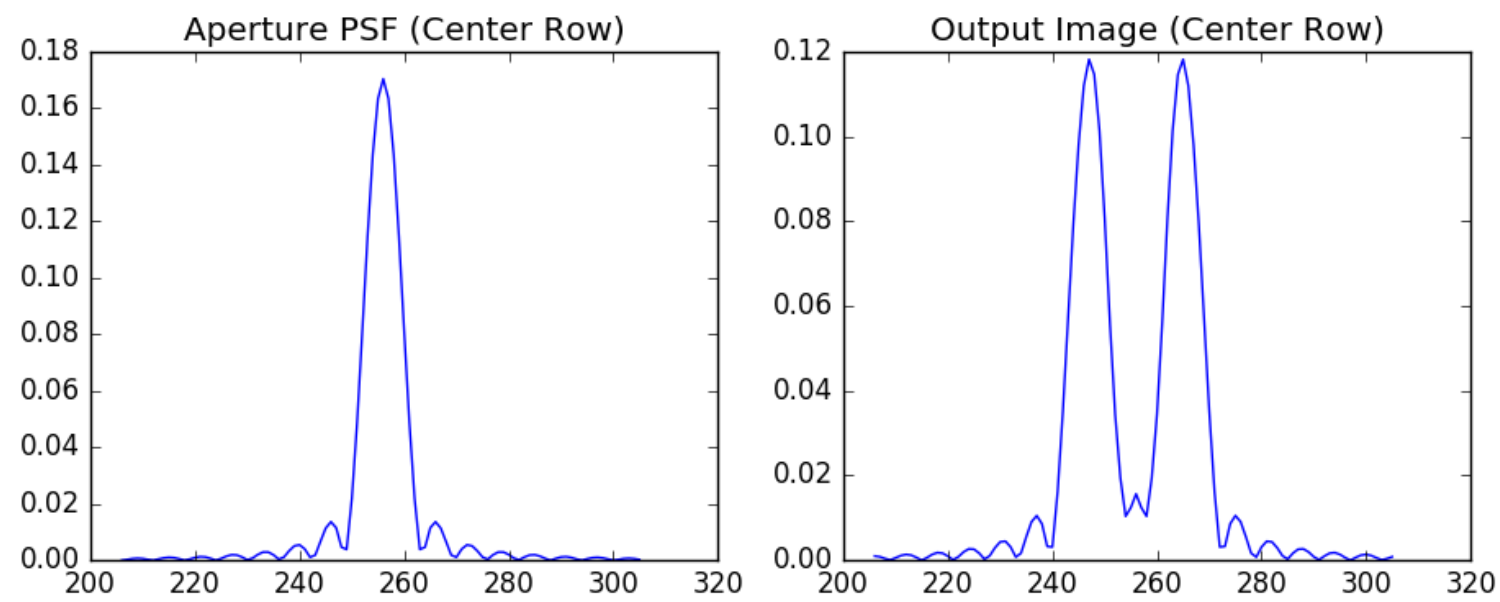

Figure 2.11: Center row of aperture diffraction image

details, a larger diameter primary mirror is required to decrease the size of the Airy disk.

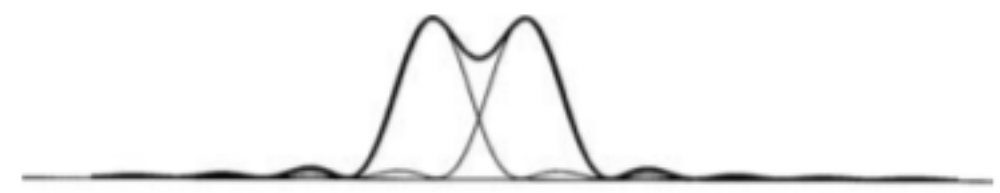

Figure 2.12: Minimum spacing as defined by Rayleigh Criterion [3]

These figures and simulations show that an ideal telescope has a limited angular resolution and that detail finer than this resolution will be lost. This angular resolution may be improved by increasing the diameter of the telescope. This implies that the diameter of the telescope may be increased indefinitely to increase the angular resolution of a telescope, allowing for studying of infinitely small objects. Unfortunately for earthbound telescopes, the distortion caused by variable density air pockets in the atmosphere often prevents reaching the theoretical diffraction limit on a large telescope. 


\subsection{Atmospheric Distortion}

All previous explanations and simulations of telescope behavior assume that the light incident on the telescope from its target is a perfect plane wave, with a flat wavefront. For space telescopes outside of the Earth's atmosphere this assumption is quite close to reality, but for telescopes on Earth's surface, beneath the atmosphere, this assumption no longer holds. Space telescopes, such as the Hubble Space Telescope, are able to create images at quite close to the diffraction limited resolution shown in the previous section. Transmitting through the atmosphere, the flat wavefront of an incident plane wave is turned into a nonuniform, bumpy wavefront that will no longer be imaged as a single, stationary Airy disk by a telescope on Earth.

Due to the sun's heating of the surface of the earth, convective cells are created in the lower atmosphere. At the boundaries of these cells, shear forces between columns of air with different velocities create turbulence, depicted in Figure 2.13. This turbulence creates cells of variable density and temperature air. Both density and temperature affect the index of refraction of a medium, so this atmospheric turbulence creates cells of variable index of refraction which light from space must propagate through to be seen by a telescope on Earth.
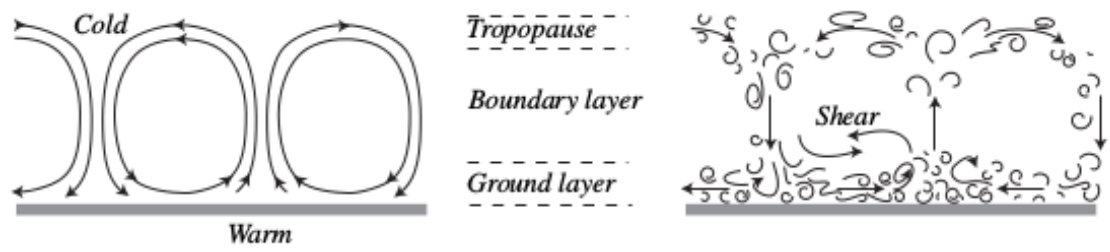

Figure 2.13: Creation of atmospheric turbulence [4] 
Having a spatially variable index of refraction means that the phase shift experienced by an incident flat wavefront is now spatially variable as well, making the transmitted wavefront no longer flat. The phase distortion of the wavefront may be numerically classified with Fried's parameter, the expected diameter over which the root-mean-square optical phase distortion is equal to one radian. From a mountaintop observatory in good seeing conditions, the Fried parameter is approximated as between $10-20 \mathrm{~cm}$. Within this diameter the wavefront may be approximated as flat [4]. If the main lens/mirror of a telescope is smaller than this diameter, an approximately flat wavefront will be received by the telescope, but this flat wavefront's incident angle will be constantly changing. This results in a small point image of the target (due to a relatively flat waveform) that is constantly moving with time (due to a time varying incident angle). This is the case when viewing stars with the naked eye, the star appears as a point source but "twinkles", showing its time varying incident angle. If the main lens/mirror of a telescope is considerably larger than this diameter, the received wavefront can't be approximated as flat, but its incident angle is relatively constant with time. This results in a more distorted image that changes with time (due to the time varying incident waveform phase shift) but remains relatively stationary in location in the image (due to constant incident angle). This is the effect that creates the speckle images that this project is concerned with. These effects are shown in Figure 2.14.

To simulate these speckle images, the atmospheric distortion must be modeled in a form compatible with the previous Fourier optics simulation. Since the incident wavefront experiences spatially varying phase shift when transmitting through the 


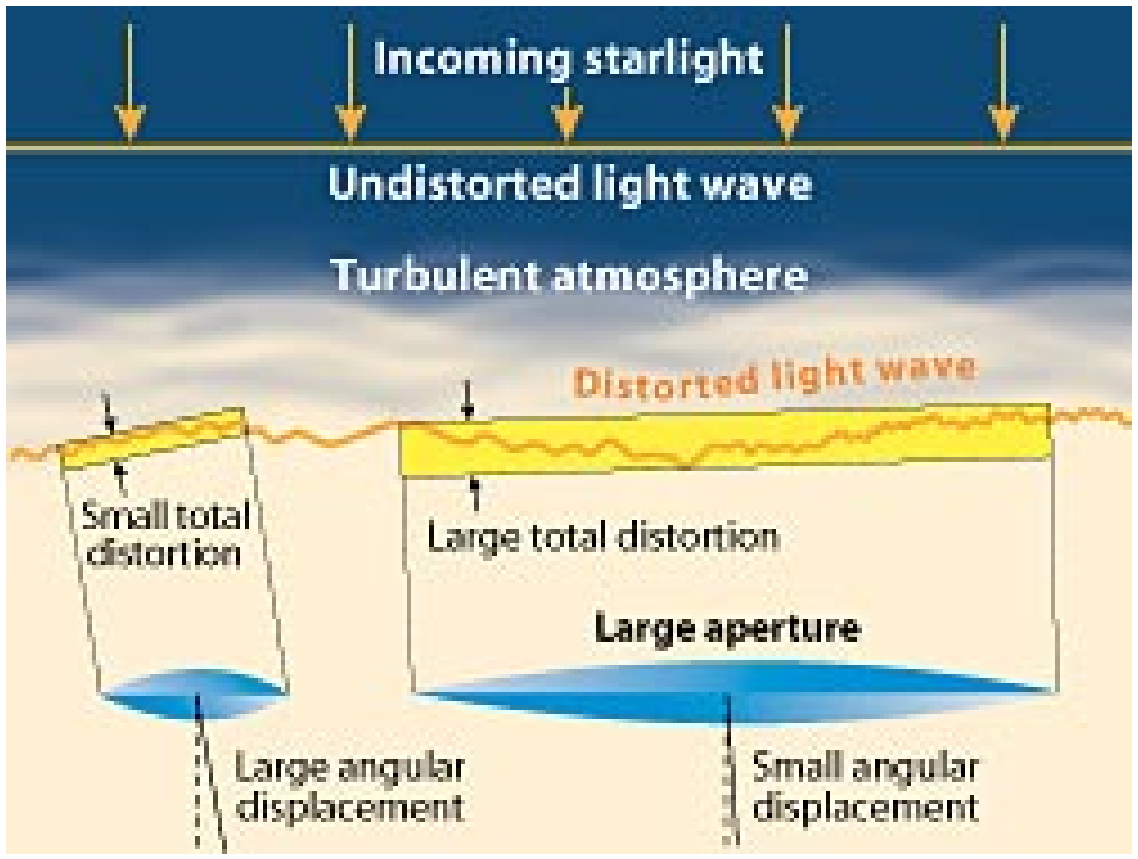

Figure 2.14: Atmospheric distortion's effect on telescope images [5]

atmosphere, the atmospheric distortion may be modeled as a phase screen. The PSF of this atmospheric screen (along with the telescope aperture) may be calculated and convolved with the input image to simulate a speckle image. The atmosphere's phase shift is modeled as a Gaussian random process with a power spectrum defined by Kolmogorov [17] [18] [19] [20] :

$$
\Phi(k)=0.023 r_{0}^{-5 / 3}|k|^{-11 / 3}
$$

The same KPNO 2.1m telescope was simulated (Appendix C) with atmospheric distortion. The complex pupil image, combining the aperture and atmospheric phase shift, is shown in Figure 2.15. The magnitude portion is the same aperture image as the previous simulation, and the phase portion is the "cloudy" atmospheric distortion. The PSF of this telescope/atmospheric distortion optical system is shown in Figure 2.16: 

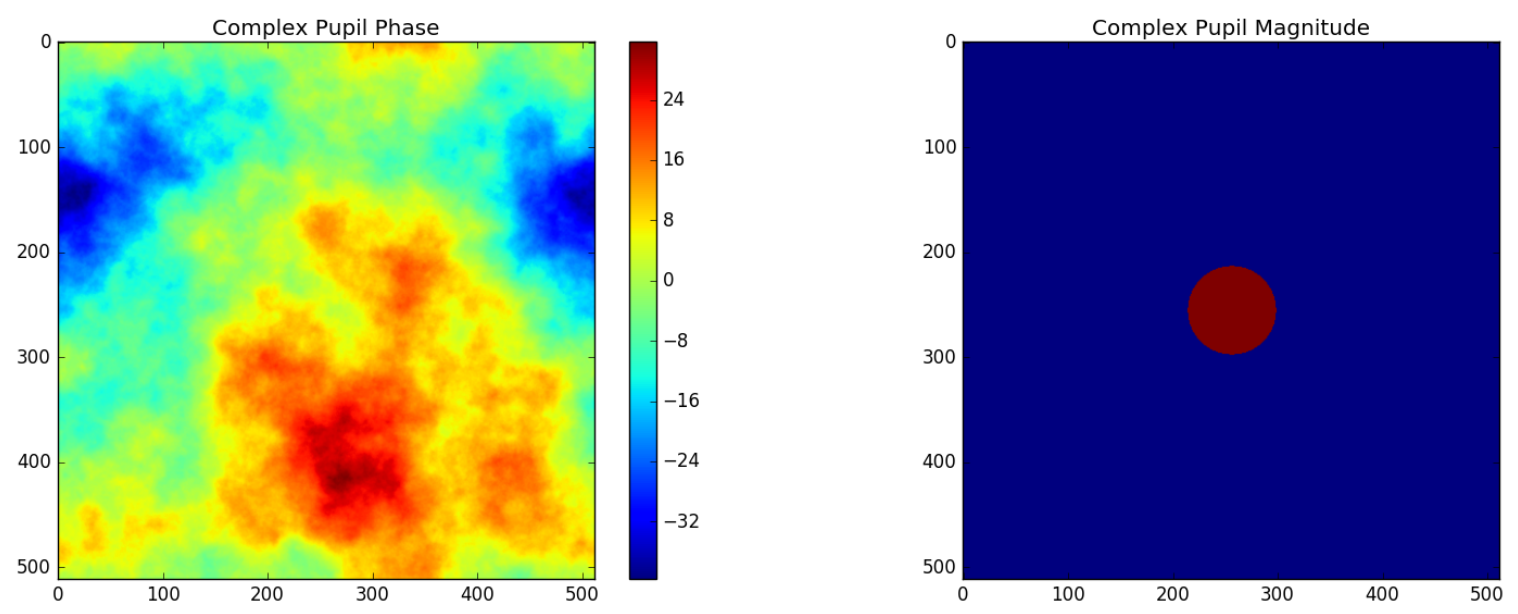

Figure 2.15: Phase and magnitude of complex pupil image

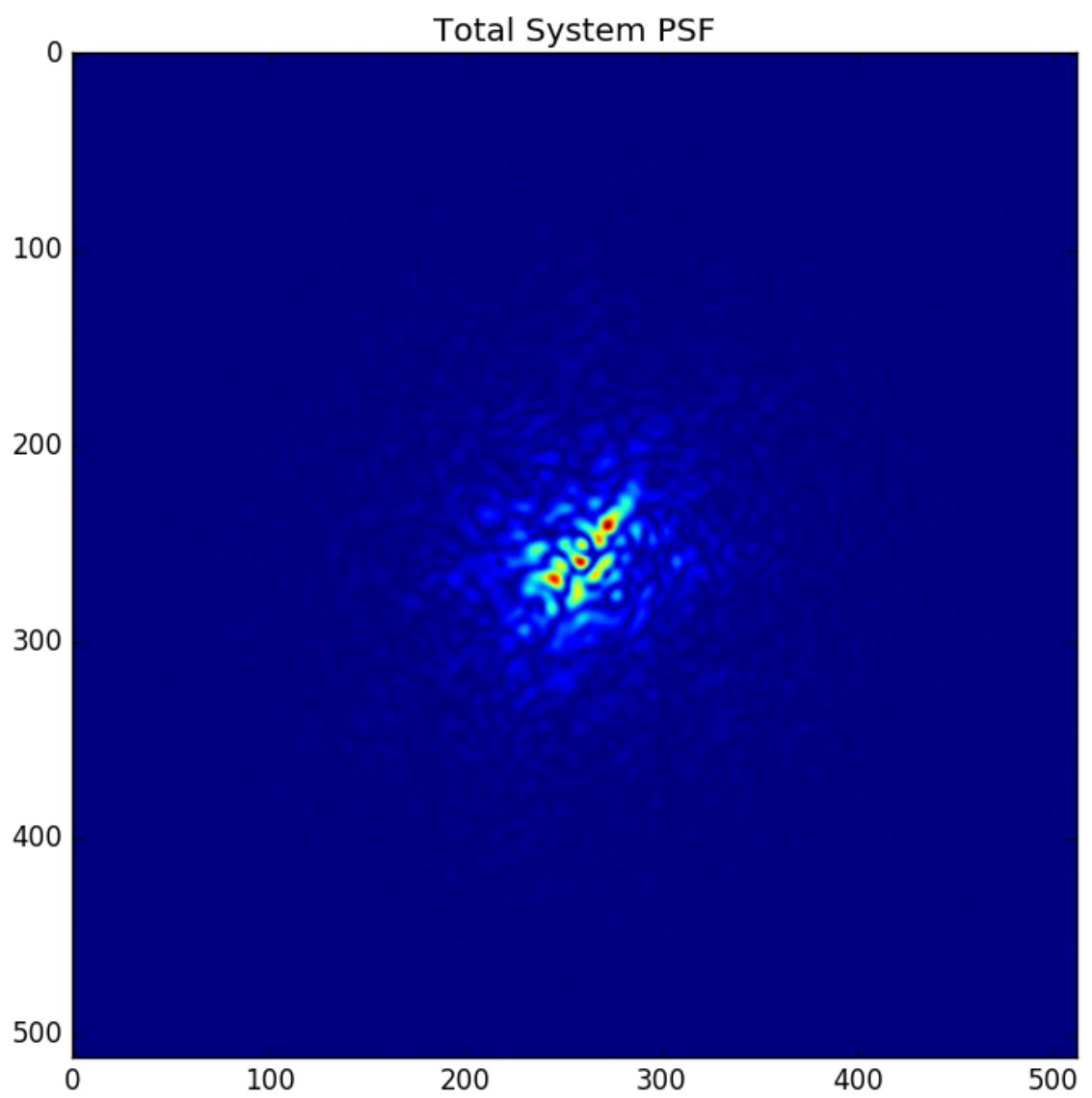

Figure 2.16: Simulated PSF through atmospheric distortion

This simulation PSF image represents a very short time exposure of a single star. In this speckle image, the size of the small features (the speckles) are affected 
by the size of the lens, while the overall envelope of the speckle pattern is affected by the atmospheric conditions. As atmospheric seeing improves, Fried's parameter increases, and this envelope decreases in size.

When observing with fast exposures on dim targets, the number of photons detected during an exposure may become low enough that photon shot noise becomes an important noise source. The arrival of a photon from a star source is a temporally random, discrete event, with each event independent from the others [21] [22]. For a dim target/short exposure, where the number of photons arriving from the source is small, this random element of the captured signal becomes very visible, causing noticeable differences between subsequent images of the same source. For a bright target/long exposure, the number of photons arriving is very large, so the random element becomes less noticeable and subsequent images are nearly the exact same.

The probability of a number of photons being captured over a time interval is modeled with a Poisson distribution:

$$
P(N)=\frac{e^{-\lambda}(\lambda)^{N}}{N !}
$$

Where: $N=$ Photons Detected; $\lambda=$ Expected Photons Detected

In a simulation not taking shot noise (or other noise sources) into account, subsequent images of a static object should be exactly the same, meaning the number of photons received at each pixel of the photo sensor are the exact same between images. So to add shot noise to a simulation like this, each pixel's new value may be calculated as a random value from a Poisson distribution, whose expected pho- 
tons detected, $\lambda$, value corresponds to the old, static pixel value. If all the old pixel values for the entire sensor are added together, this gives the total number of photons expected to arrive at the sensor. Scaling the entire image by a constant scales the number of photons appearing in the new shot noise image.

An important part of considering noise in simulations and measurements is knowing when to consider the effects of the noise significant or not. Because this noise is modeled as a Poisson distribution, the relationship between the standard deviation and mean are known as $\sigma^{2}=\mu$ [4]. This means the signal to noise ratio may be calculated as:

$$
S N R=\frac{\mu}{\sigma}=\frac{N}{\sqrt{N}}=\sqrt{N}
$$

This shows that as the number of photons received increases, the SNR increases, and the effects of noise are less visible. So for suitably bright objects, this effect may be disregarded. This may be seen in the simulation image shown in Figure 2.17, comparing images from both the noise free image and noisy images with different numbers of photons received.
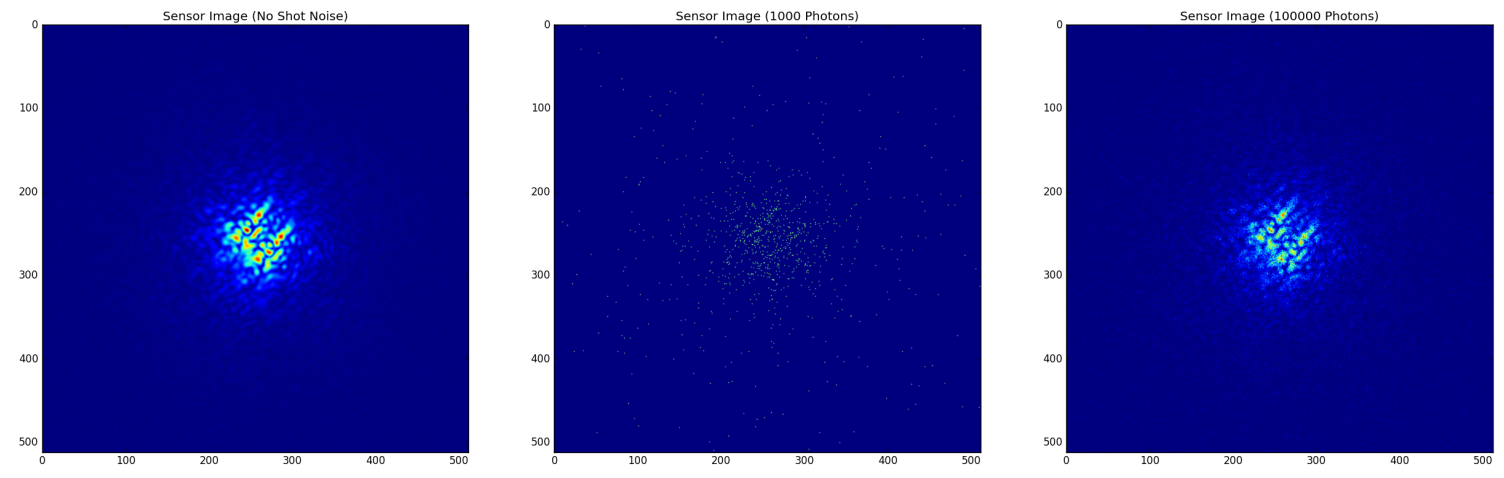

Figure 2.17: Adding shot noise to simulated image 
For 1000 photons detected, the image barely resembles its uncorrupted verison, indicating a low SNR. But for 100E3 photons incident, the image begins to look very similar to the uncorrupted version, indicating a higher SNR. A degraded SNR may limit the precision of measurements made by the astronomer, so there is a fundamental limit to how dim the target may be or how fast the shutter speed may be.

In addition to photon shot noise, there are several additive noise sources that may contribute to the final image such as CCD read noise (both on and off the sensor chip) and electronic interference. These noise sources are dependent on factors such as the design of the camera/image sensor, the temperature of the sensor, and the pixel read speed. These noise sources are modeled as additive gaussian white noise functions [23] [24] [25]. That is, each pixel has an independent gaussian random value added to it. A comparison of a simulated reference star image with and without Gaussian read noise is shown in Figure 2.18, where number of electrons incident $=50 * 10^{3}$ and additive read noise variance $=3$ photons.

This theory and simulation shows that when observing with a large telescope from beneath the atmosphere, such as the KPNO $2.1 \mathrm{~m}$ telescope, the quality of the images captured is limited by the atmospheric seeing and noise sources, rather than just the size of the primary optic. This means that for closely spaced visual binary stars, the raw images captured rarely show two distinct objects easily identified as two stars. In order to view these binary stars, speckle interferometry techniques may be employed. 

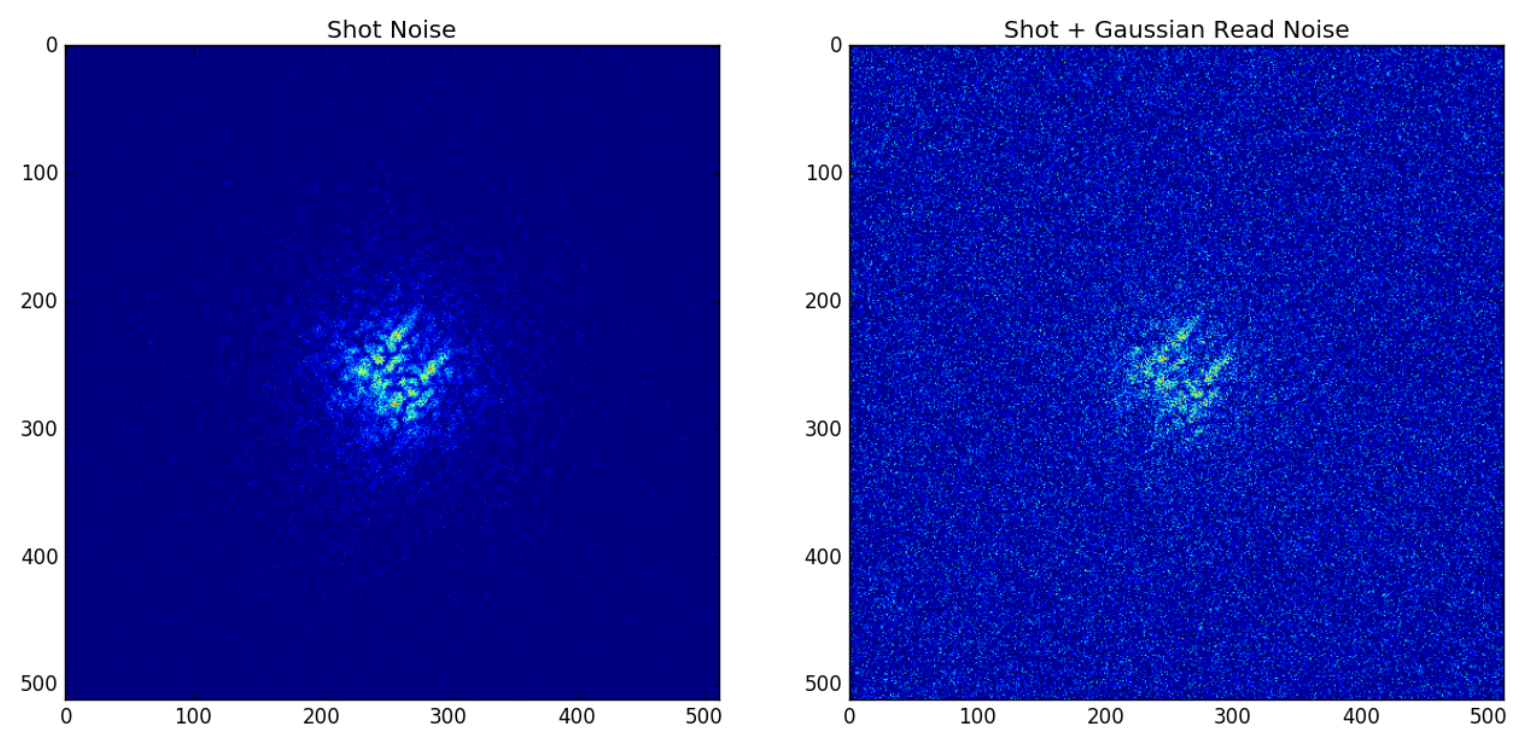

Figure 2.18: Adding Gaussian read noise to simulated image 


\section{LABEYRIE DECONVOLUTION}

\subsection{Labeyrie's Findings}

Labeyrie took advantage of the interference properties of light in order to recover diffraction limited information from the image. Because his important findings were in 1970, many of his "computations" were performed optically rather than computationally.

When a close binary star is viewed with an eyepiece or a long exposure photograph, the high speed changes in the speckle pattern are integrated and blurred together, producing the large atmospheric seeing limited pattern. When the same star is viewed with a very short exposure photograph (10-15ms), some high spatial frequency data is visible in the form of the bright speckles. These differences between long and short exposure image can be seen in Figure 3.1.
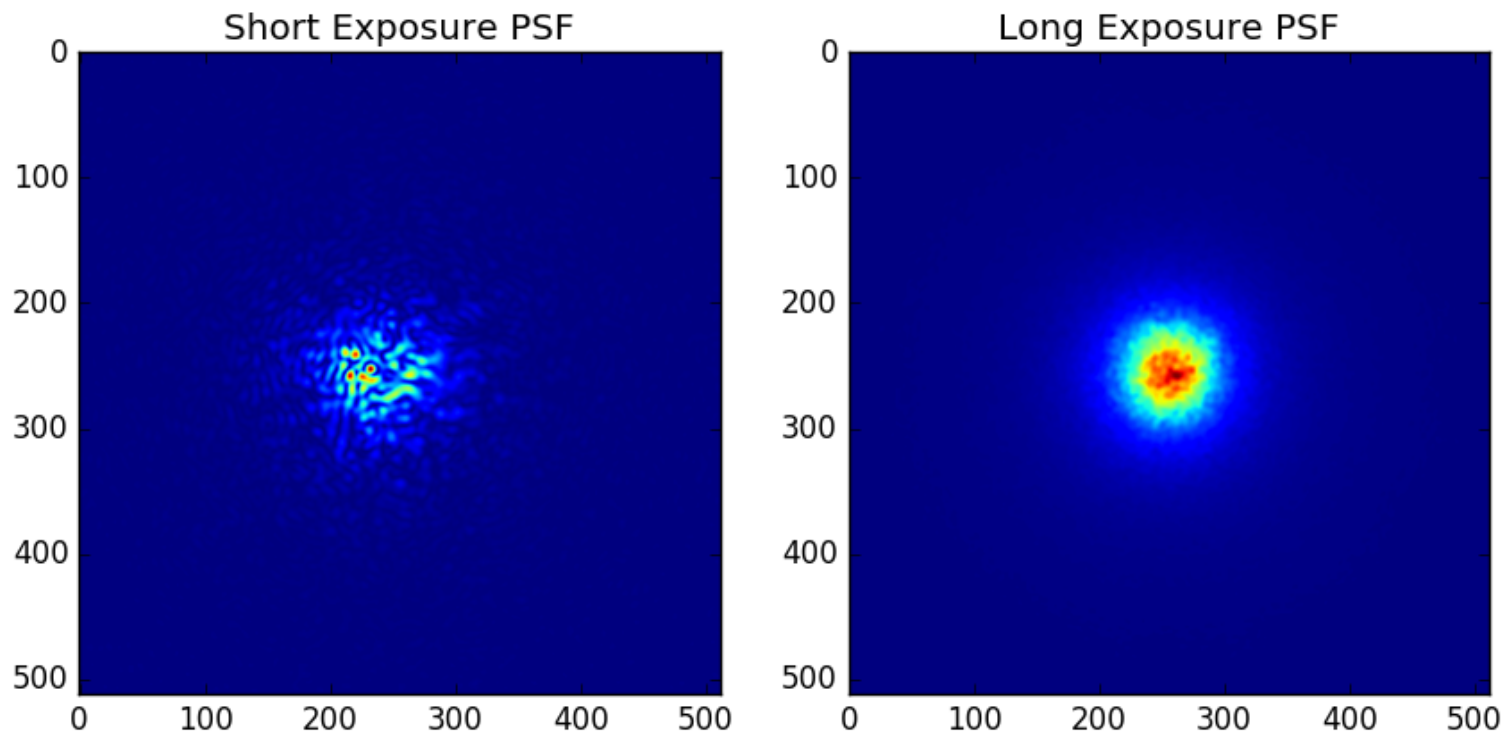

Figure 3.1: Short vs long exposure times through atmospheric distortion 
In all the previous simulations, the assumption has been made that the targets are all within the isoplanatic patch. The isoplanatic patch is defined as the area over the sky where the PSF is constant [26]. This isoplanatic patch is often estimated to be around 5 arcsec in good seeing conditions for visible light [4]. If the primary and secondcd ary of a binary star are located within this isoplatic patch, the short exposure observed image will be a superposition of two shifted PSFs, one for each star. This can be seen in the first row of Figure 3.2. The atmospheric distortion simulations for the initial explanation of this theory leave out the noise sources to keep equations simple.

So with short exposure images of binary stars within the isoplanatic patch, higher frequency diffraction limited information is preserved and may be recovered. Labeyrie observes the Fraunhofer diffraction pattern generated by these binary star images to have interference fringes due to their inherent periodicity [27][28]. While this information is not enough to totally reconstruct the original image, Labeyrie notes that it is sufficient to extract data on the separation distance and angle of the binary star.

\subsection{Theory}

The explanation of the formation of these fringes can be done using the OTF theory presented in Section 2.3 [27]. Where Labeyrie used lasers and screens to create Fraunhofer diffractions, Fourier Transforms/FFTs may be used to create very similar images. 

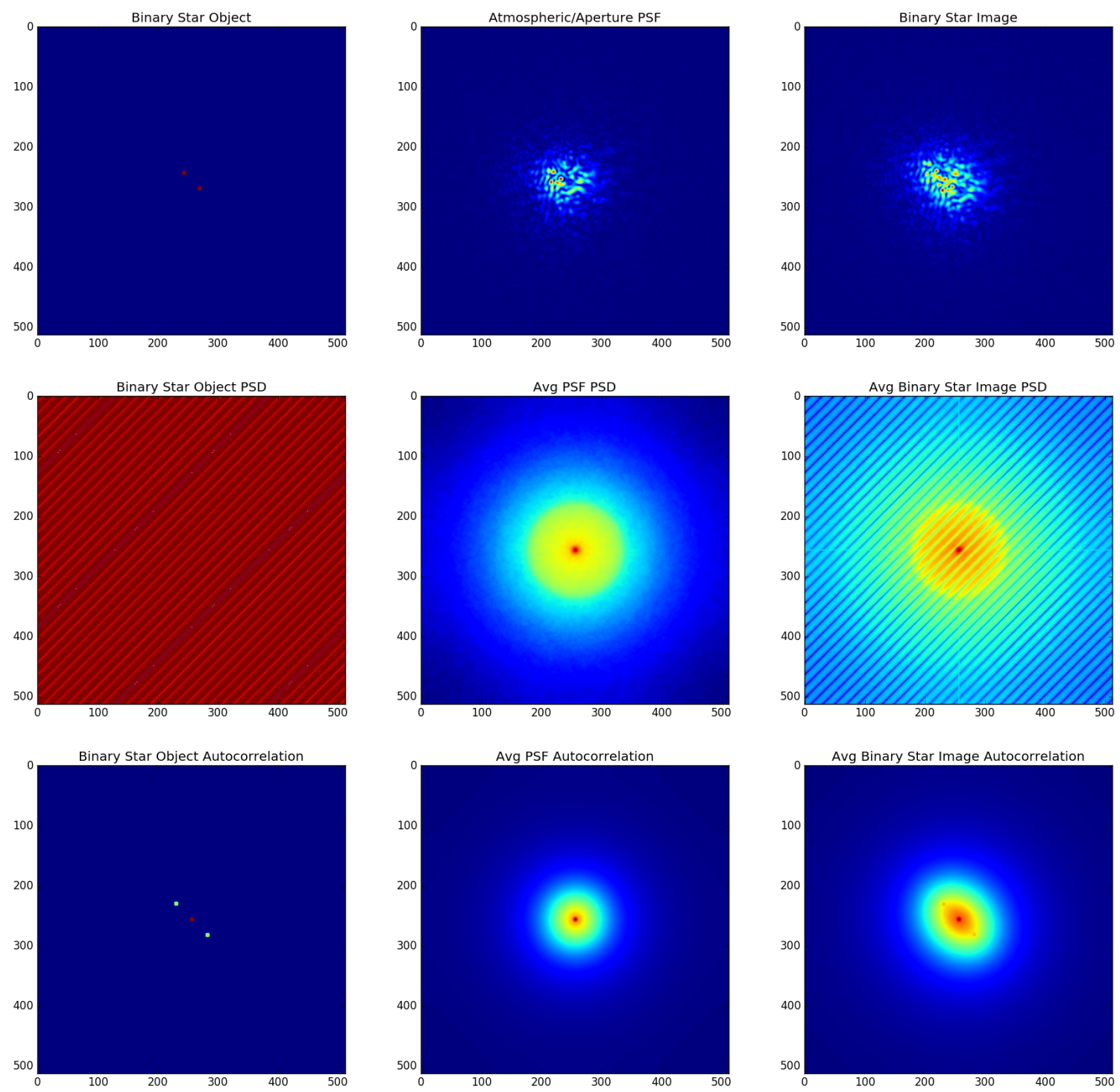

Figure 3.2: Average PSD and autocorrelation of binary star images

The object of study in this case is a binary star, and its spatial intensity is represented by $o(x, y)$. The image created by the optical system viewing this object is represented as $i(x, y) . i(x, y)$ may be calculated as the convolution of the optical system's PSF, $p(x, y)$, with $o(x, y)$.

$$
o(x, y) * p(x, y)=i(x, y)
$$


Viewing the Fraunhofer diffraction patterns of these images generated optically is equivalent to viewing the squared magnitude (intensity) of the Fourier Transform of these images, also known as the Power Spectral Density (PSD). The PSD of the image may be calculated as shown below, where $I(u, v), P(u, v)$, and $O(u, v)$ are the Fourier Transforms of the image, PSF, and object respectively:

$$
|O(u, v)|^{2} \cdot|P(u, v)|^{2}=|I(u, v)|^{2}
$$

With data from previous simulations, this relationship between the original image and their PSDs can be shown graphically in the first two rows in Figure 3.2.

If the object being observed is a binary star, the object is represented ideally as two point sources, which creates a Fraunhofer diffraction pattern of fringes. The angle and separation of these fringes hold the important astrometric information about the binary star target. These fringes are still visible in the PSD of $I(u, v)$, so the information is still present in the speckle image.

Since each image contains an element of random noise from the atmosphere, the signal to noise ratio of the PSD may be improved by averaging many image PSDs together. The atmospheric distortion is a random process that varies between each image, but some of its properties (size and number of Fried Parameter seeing cells) are relatively constant with time. These PSDs are averaged below. Note that $\langle O(u, v)\rangle=O(u, v)$ because the object is a constant.

$$
|O(u, v)|^{2} \cdot<|P(u, v)|^{2}>=<|I(u, v)|^{2}>
$$

If this is all the information the astronomer has, useful information may be calculated from this PSD. If the inverse Fourier Transform is taken, the separa- 
tion angle and distance of the object may sometimes be determined, depending on the quality of the seeing. The inverse Fourier Tranform of a PSD function is the autocorrelation of the original spatial signal. This is shown below, where $R_{f f}(x, y)=$ autocorrelation of $\mathrm{f}(\mathrm{x}, \mathrm{y})$ function

$$
\begin{gathered}
\mathcal{F}^{-1}\left\{|O(u, v)|^{2} \cdot<|P(u, v)|^{2}>=<|I(u, v)|^{2}>\right\} \\
R_{o o}(x, y) *<R_{p p}(x, y)>=<R_{i i}(x, y)>
\end{gathered}
$$

The result of these calculations is the average autocorrelation of each binary star image. This average autocorrelation image is the convolution of the autocorrelation of the object (the desired information, constant with time) with the average autocorrelation of the aperture/atmospheric PSF (undesirable information, different in each image). This relationship is shown in the bottom row of Figure 3.2

The average binary star image autocorrelation is the most useful image from these calculations. The noticeable features are the large primary lobe in the center of the image with two smaller secondary lobes symmetrically positioned around this primary lobe. The angle and distance of the separation between the primary and secondary lobes indicates the angle and distance of the separation between the primary and secondary stars of the original binary star.

For binary stars with large enough separation angle, this average image autocorrelation can provide the astrometric information desired, as the primary and secondary lobes may be resolved. However, for closely spaced binary stars, the primary and secondary lobes begin to overlap and combine. As spacing decreases further, eventually the lobes will be impossible to resolve and the desired astromet- 
ric information can no longer be gathered. To get around this problem, a reference star may be used to deconvolve the atmospheric PSF from the binary speckle images.

A reference star is a single star located within the same isoplanatic patch as the binary star is located. Observing this reference star will give the PSF of the same atmospheric distortion that the binary star is viewed through. Since the properties of this atmospheric distortion are relatively constant over time, the averaged PSD of the reference star's PSF should be very similar to the averaged PSD of the atmospheric PSF present in binary star images. Rearranging the average image PSD equation gives:

$$
|O(u, v)|^{2}=\frac{<|I(u, v)|^{2}>}{<|P(u, v)|^{2}>}
$$

Since the $<|P(u, v)|^{2}>$ term can be found by observing a reference star, this equation shows that the atmospheric distortion may be theoretically removed from the average binary star image PSD, giving the PSD of the object. This process of using the reciprocal of the degrading function is known as inverse filtering [23]. As may be seen in Figure 3.3, the deconvolved PSD calculated with this inverse filter appears as a nearly perfect set of fringes, with no shaping from the telescope aperture or atmosphere like in the average binary star image PSD. The inverse Fourier Transform of this deconvolved PSD gives the autocorrelation of the binary star object, without any of the telescope aperture or atmospheric effects shown in the binary star image autocorrelation. The result is that the lobes of the deconvolved autocorrelation are sharper and easily resolved compared to the binary star image 
autocorrelation. In fact, this deconvolved autocorrelation looks nearly identical to the input image, suggesting that the information has been entirely recovered.
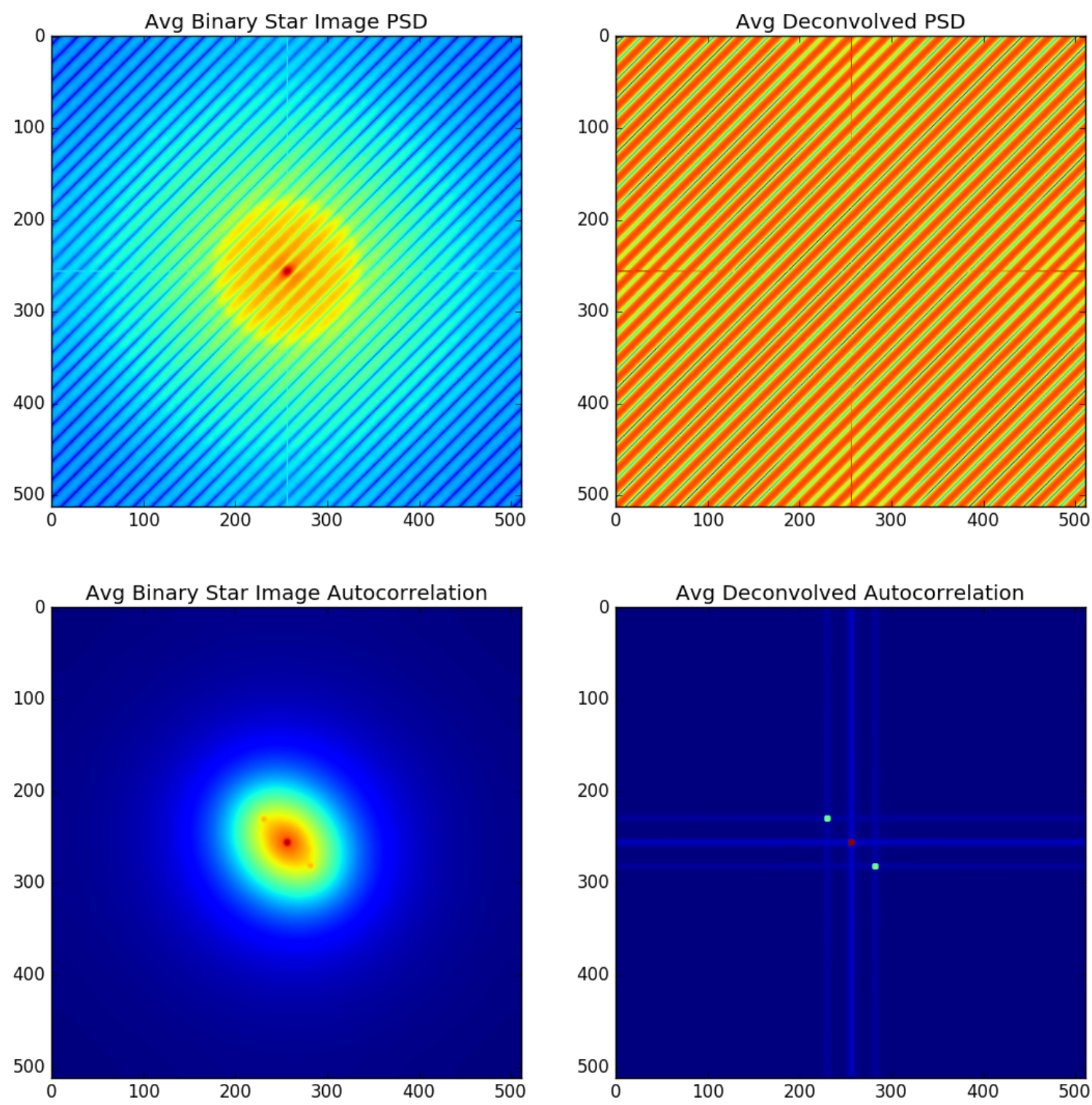

Figure 3.3: Recovered binary star object PSD/autocorrelation

Unfortunately, this simulation has been done without any noise present, which is not a realistic condition. As explained previously, both shot and gaussian noise can be expected in the images of both the binary and reference stars. This noise is not band limited (unlike the optical information capture by the telescope), but 
is present at all spatial frequencies. The reference star PSD contains most of its energy in two areas, shown in Figure 3.4, where the noiseless reference star PSD is displayed logarithmically. The optical system's components act as spatial filters, both atmospheric distortion and aperture being spatial lowpass filters. The energy transmitted by atmospheric distortion is concentrated at low frequencies, resulting in the primary, central peak in the PSD. The energy transmitted by the optical system's finite sized aperture has a wider bandwidth and is located in the PSD's wider lobe.

Due to this optical system's low-pass nature, the optical energy at very high frequencies, beyond the cutoff frequency of the aperture, is much lower than the energy at lower frequencies. To make the simulation more realistic, small amounts of wideband noise are added. At low frequencies, where the majority of signal energy is located, the signal-to-noise ratio remains acceptable and isn't significantly different. However, at very high frequencies, because of the lack of signal energy, the signal-to-noise ratio becomes very poor. When performing deconvolution through inverse filtering this can lead to this high frequency noise energy dominating low frequency signal energy in the deconvolved PSD, as shown below in Figure 3.5. This means that the signal in the deconvolved autocorrelation (seen as lobes) is dominated by noise (in the form of tall and sharp peaks), so image contrast is very low and little information can be retrieved.

This effect may be shown mathematically. The noise added to the captured image is represented as $N(u, v)$ in the frequency domain. In the presence of noise, 


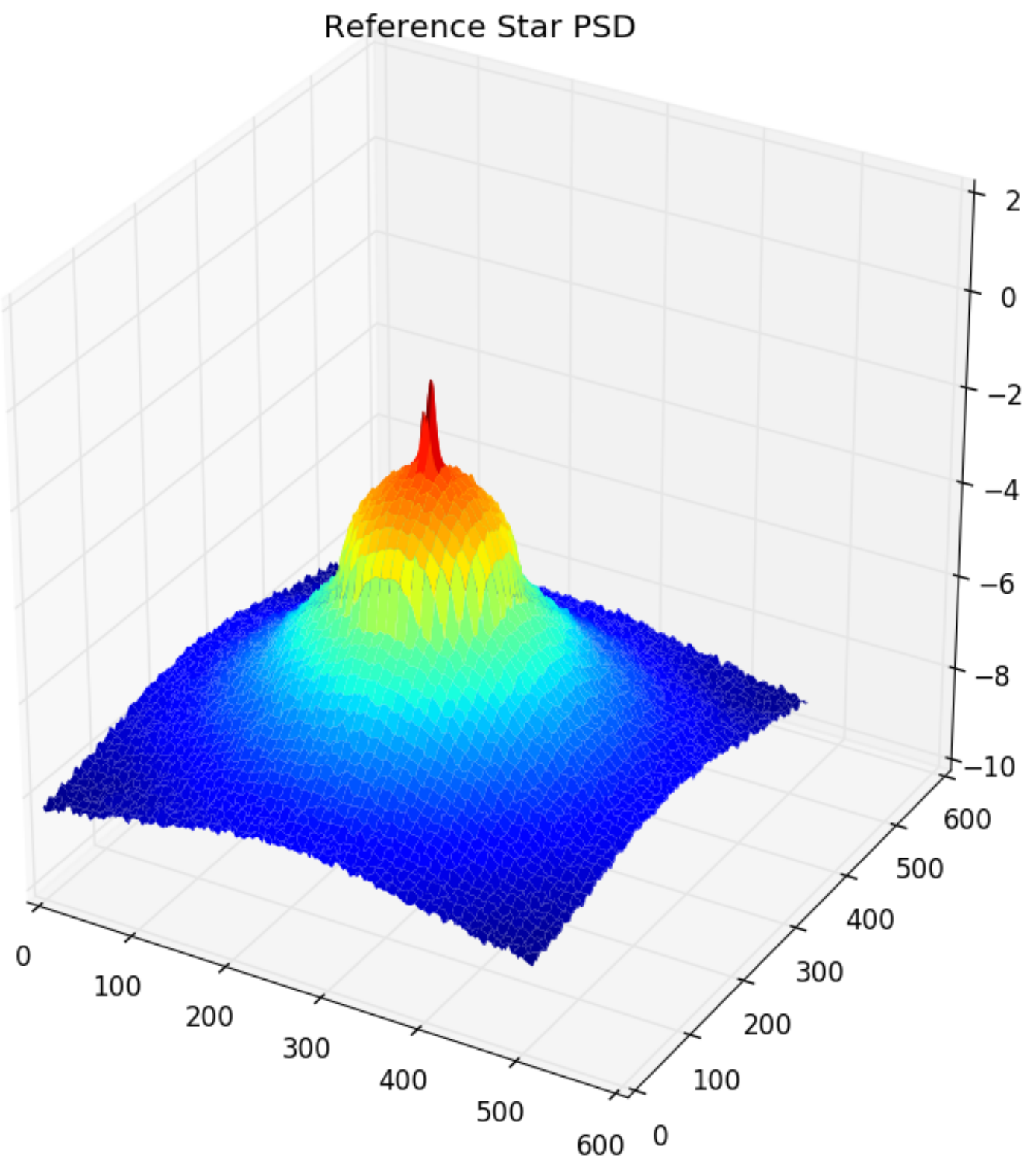

Figure 3.4: Sources of signal energy in noiseless PSD

the frequency domain image captured by the telescope may be described as:

$$
O(u, v) \cdot P(u, v)+N(u, v)=I(u, v)
$$



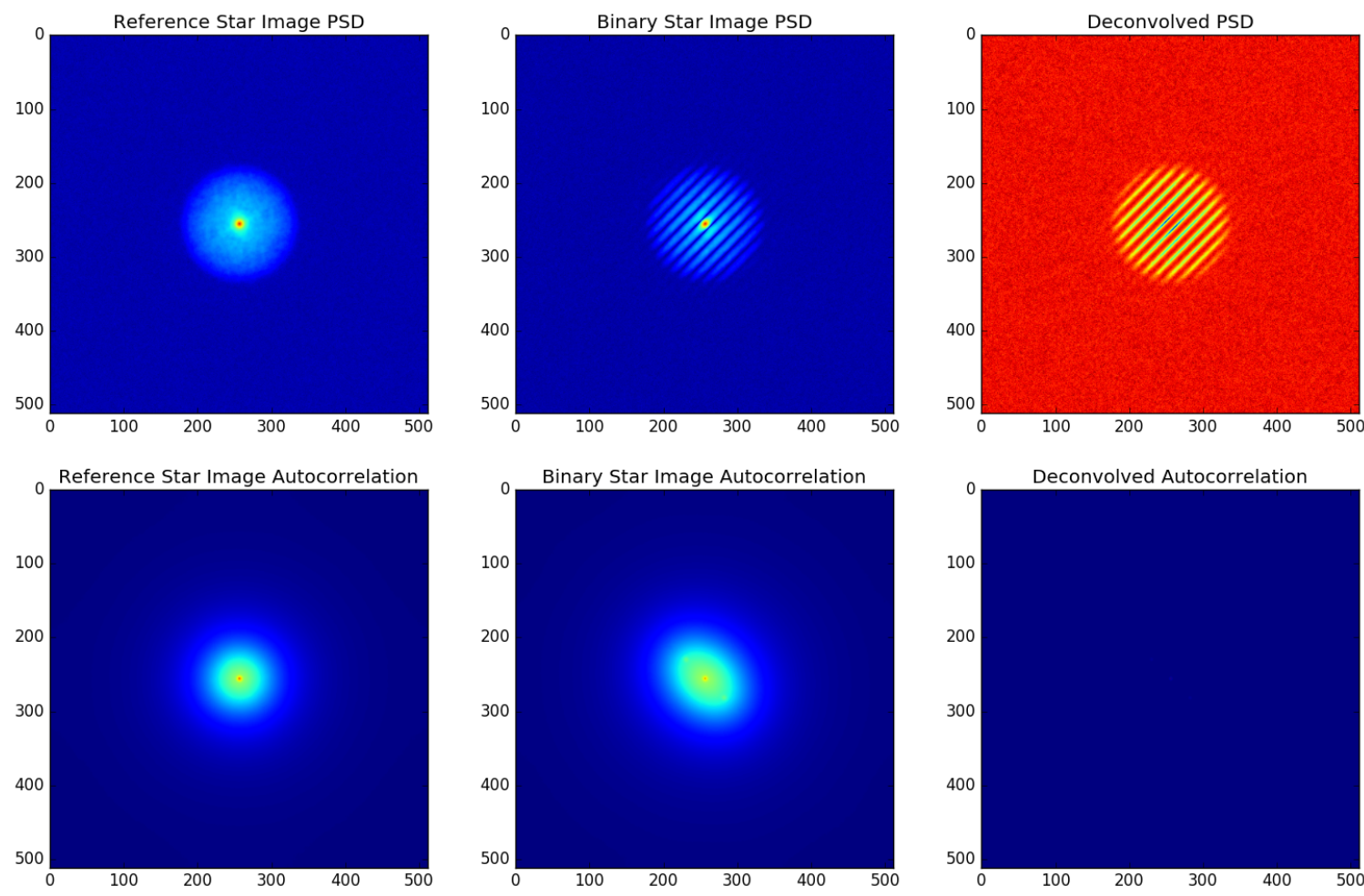

Figure 3.5: Domination of high frequency noise in inverse filtering

If inverse filtering is used in an attempt to deconvolve the reference star from the observed binary star image, the following results:

$\hat{I}(u, v)=\frac{O(u, v) \cdot P(u, v)+N(u, v)}{P(u, v)}=\frac{O(u, v) \cdot P(u, v)}{P(u, v)}+\frac{N(u, v)}{P(u, v)}=O(u, v)+\frac{N(u, v)}{P(u, v)}$

Like before, the desired uncorrupted object $O(u, v)$ is recovered, but this time, the wideband noise value is also present. Because the spectrum of the undesirable system PSF $P(u, v)$ is lowpass in nature, its inverse becomes highpass shaped. This has the effect of attenuating noise at low frequencies (where the majority of the signal energy is located) and amplifying it at high frequencies (where little to no signal energy is present). This leads to the dominance of high frequency noise in the deconvolution in the presence of noise shown in Figure 3.5. This shows that when dealing with real data with noise, inverse filtering is rarely effective. This 
noise corrupted signal and recovery model does not perfectly apply to Labeyrie's deconvolution, as Labeyrie's deconvolution uses average PSDs, while this model uses the frequency domain of a single image, but the general behavior still applies.

While the inverse filter has been shown to be ineffective in the presence of noise, the Wiener filtering deconvolution technique considers noise in its derivation and still gives an accurate deconvolution estimate [23] [29]. The equation for this filter is shown below:

$$
\hat{F}(u, v)=\left[\frac{1}{H(u, v)} \frac{|H(u, v)|^{2}}{|H(u, v)|^{2}+S_{n}(u, v) / S_{f}(u, v)}\right] G(u, v)
$$

Where

$$
\begin{gathered}
\hat{F}(u, v)=\text { Recovered Signal, } H(u, v)=\text { Degrading function } \\
\qquad(u, v)=\text { Degraded image } \\
S_{n}(u, v)=\text { Noise PSD, } S_{f}(u, v)=\text { Uncorrupted signal PSD }
\end{gathered}
$$

It may be noted that

$$
S_{n}(u, v) / S_{f}(u, v)=1 / S N R(u, v)
$$

In a noisy environment, in regions where the signal energy dominates the noise energy, the Wiener filter behaves as the inverse filter.

$$
\begin{gathered}
\operatorname{SNR}(u, v) \approx \infty \\
\hat{F}(u, v)=\left[\frac{1}{H(u, v)} \frac{|H(u, v)|^{2}}{|H(u, v)|^{2}+1 / \infty}\right] G(u, v) \\
\hat{F}(u, v)=\left[\frac{1}{H(u, v)} \frac{|H(u, v)|^{2}}{|H(u, v)|^{2}+0}\right] G(u, v) \\
\hat{F}(u, v)=\left[\frac{1}{H(u, v)}\right] G(u, v)
\end{gathered}
$$


In regions where the noise energy dominates, the Wiener filter attenuates, suppressing the undesirable noise.

$$
\begin{gathered}
\operatorname{SNR}(u, v) \approx 0 \\
\hat{F}(u, v)=\left[\frac{1}{H(u, v)} \frac{|H(u, v)|^{2}}{|H(u, v)|^{2}+1 / 0}\right] G(u, v) \\
\hat{F}(u, v)=\left[\frac{1}{H(u, v)} \frac{|H(u, v)|^{2}}{|H(u, v)|^{2}+\infty}\right] G(u, v) \\
\hat{F}(u, v)=0
\end{gathered}
$$

In the context of Labeyrie's deconvolution, this model for a noise corrupted signal doesn't apply perfectly. This is because rather than deconvolving a degrading image from a single degraded image, we are attempting to deconvolve an average modulus squared degrading image from an average modulus square degraded image. This means that the Wiener filter will not perform exactly is it was designed to do, but the basic concepts of inverse filtering in areas of high SNR and attenuating in areas of low SNR still apply. To apply the Wiener filter to Labeyrie's deconvolution, its variables are set as:

$$
\begin{gathered}
\hat{F}(u, v)=\hat{O}(u, v), H(u, v)=<|P(u, v)|^{2}> \\
G(u, v)=<|I(u, v)|^{2}> \\
S_{n}(u, v)=<|N(u, v)|^{2}>^{2}, S_{f}(u, v)=<|O(u, v)|^{2}>^{2}
\end{gathered}
$$

So the Wiener filter becomes

$$
<|\hat{O}(u, v)|^{2}>=\left[\frac{<|P(u, v)|^{2}>^{2}}{<|P(u, v)|^{2}>^{2}+<|N(u, v)|^{2}>^{2} /<|O(u, v)|^{2}>^{2}}\right] \frac{<|I(u, v)|^{2}>}{<|P(u, v)|^{2}>}
$$


Using the ideal Wiener filter requires full knowledge of the PSD of the noise as well as the uncorrupted signal. For the presented model including additive gaussian white noise and shot noise, the distribution of noise energy is uniform over spatial frequency spectrum [30]. This means that a constant may be used for the noise energy variable $N(u, v)$. Because the PSD of the uncorrupted signal is often unknown, a common simplification is to use a constant value for the signal PSD $O(u, v)$ as well [23], resulting in the following simplification of the Wiener filter:

$$
<|\hat{O}(u, v)|^{2}>=\left[\frac{<|P(u, v)|^{2}>^{2}}{<|P(u, v)|^{2}>^{2}+K}\right] \frac{<|I(u, v)|^{2}>}{<|P(u, v)|^{2}>}
$$

As $K$ is increased, more of the central lobe is attenuated, along with increased attenuation of noise. An excessively high $K$ value will attenuate high frequency components of the desired signal, which causes the autocorrelation's lobes to be wider, preventing resolving of closely spaced binaries. A value of $K$ is chosen experimentally to give the greatest contrast between the desired, diffraction limited information and the background noise while still giving a sharp autocorrelation image. The high signal energy regions where inverse filtering is performed is shown in Figure 3.6. The results of this simplified Wiener filtering is shown in Figure 3.7.

For this simulated binary and reference star data, this simplified Wiener filter very effectively removes the atmospheric distortion frequency components while keeping wideband noise attenuated to reasonable levels, recovering a close to ideal diffraction limited image of the binary star.

When processing data from KPNO, additional high frequency noise was observed in calculated PSDs, potentially from electrical interference. This noise was 


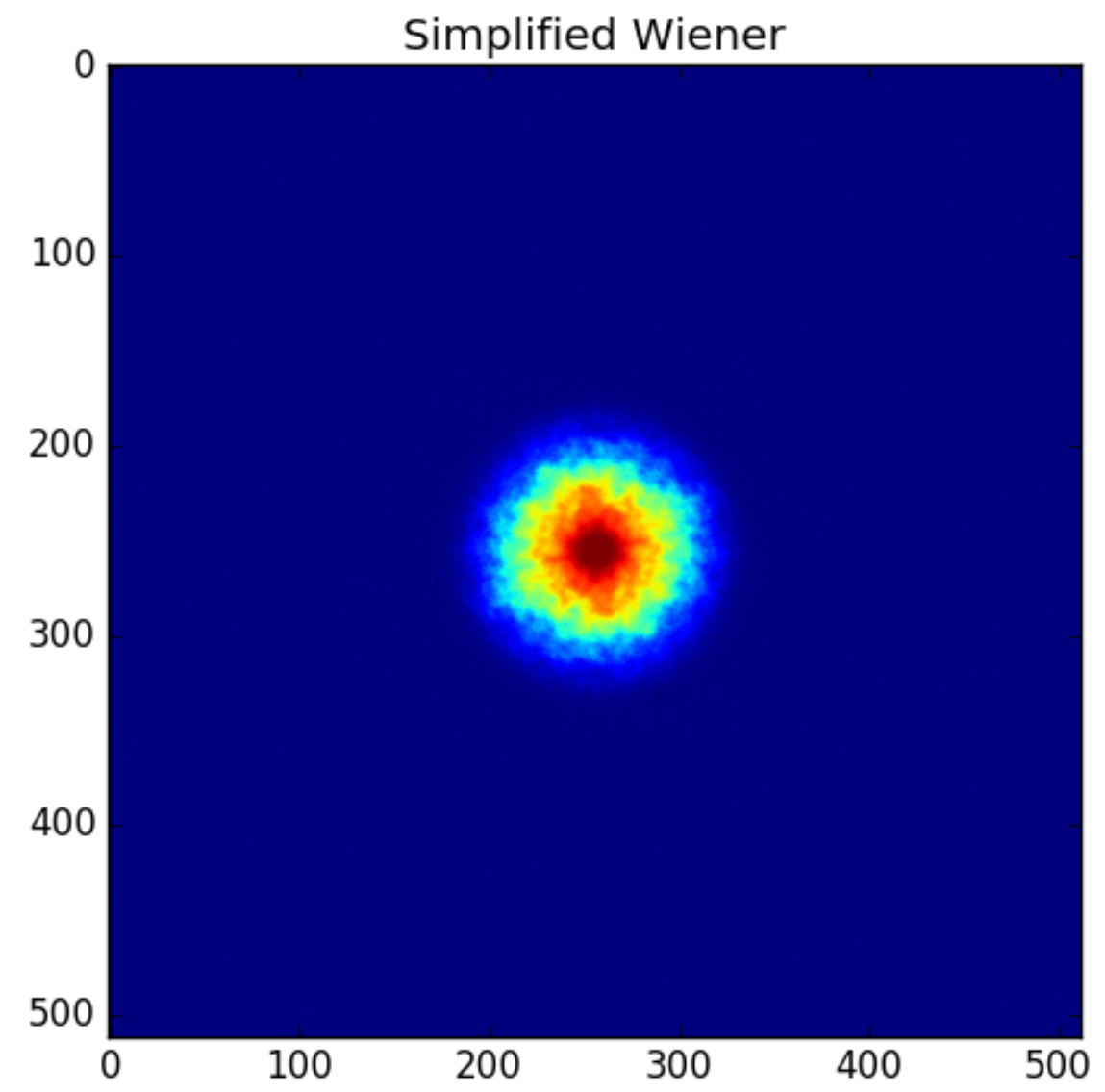

Figure 3.6: Wiener filter inverse filter region
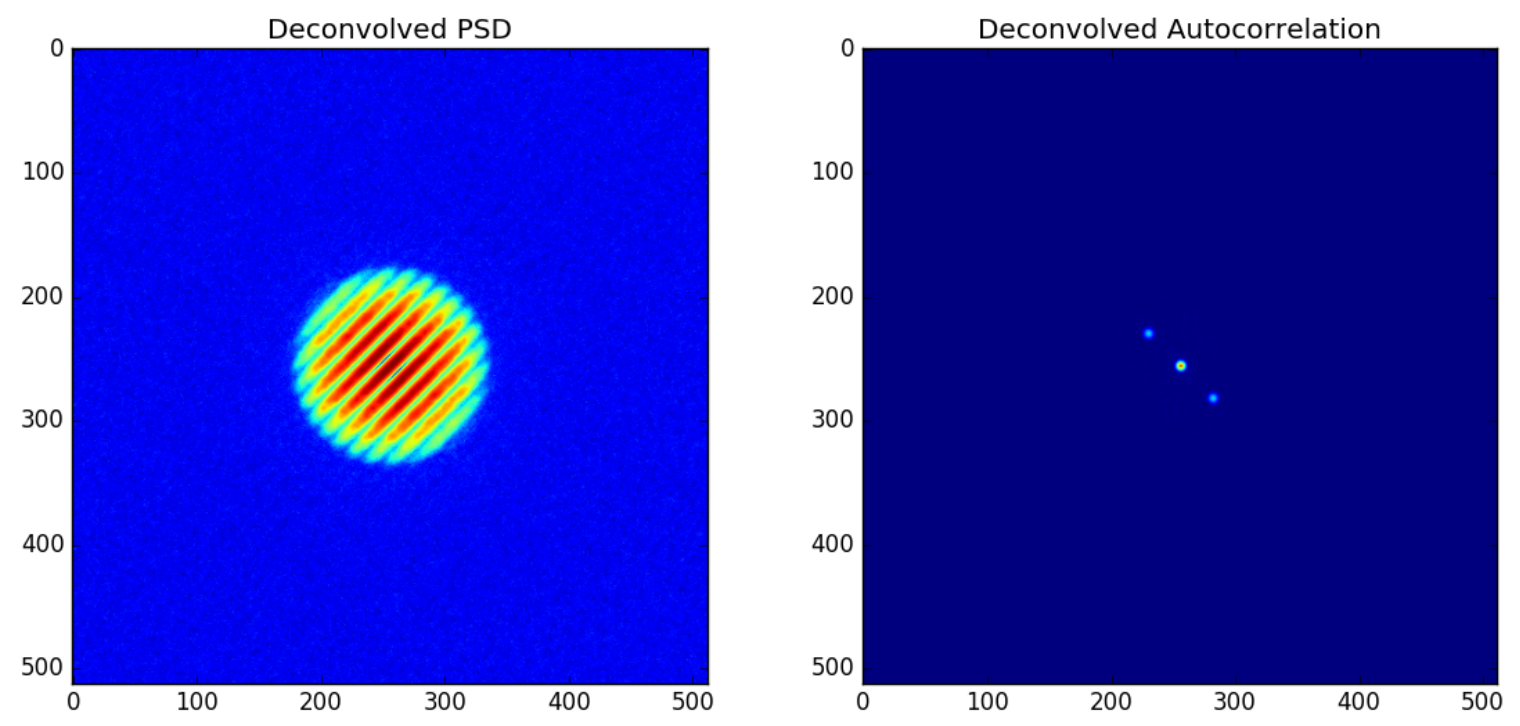

Figure 3.7: Results from simplified Wiener filter deconvolution 
not present in simulated data, and so extra measures were taken to deal with this noise. Example data of binary and reference star images along with their average PSDs is shown in Figure 3.8.
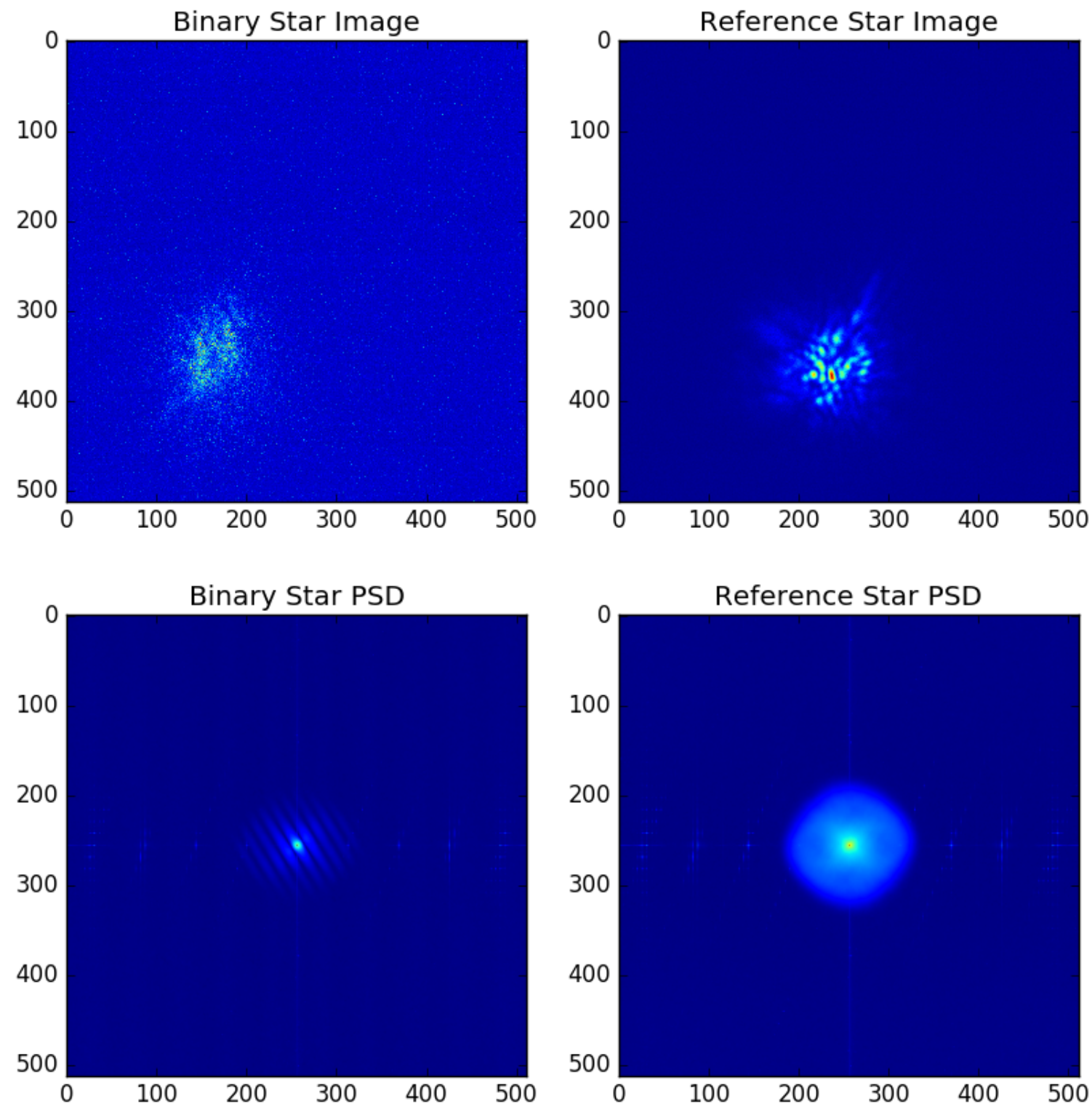

Figure 3.8: Binary and Reference star data from KPNO

Noise is visible along the axes of the reference star PSD. When deconvolving with the simplified Wiener filter discussed previously, this noise remains visible in the deconvolved PSD, seen in Figure 3.9. Trying to adjust the constant $K$ variable 
to improve the quality of the deconvolution is ineffective, increasing $K$ attenuates noise but also the signal. Setting $K$ for sharp lobes leaves high frequency noise in the image, seen in the background of the image.
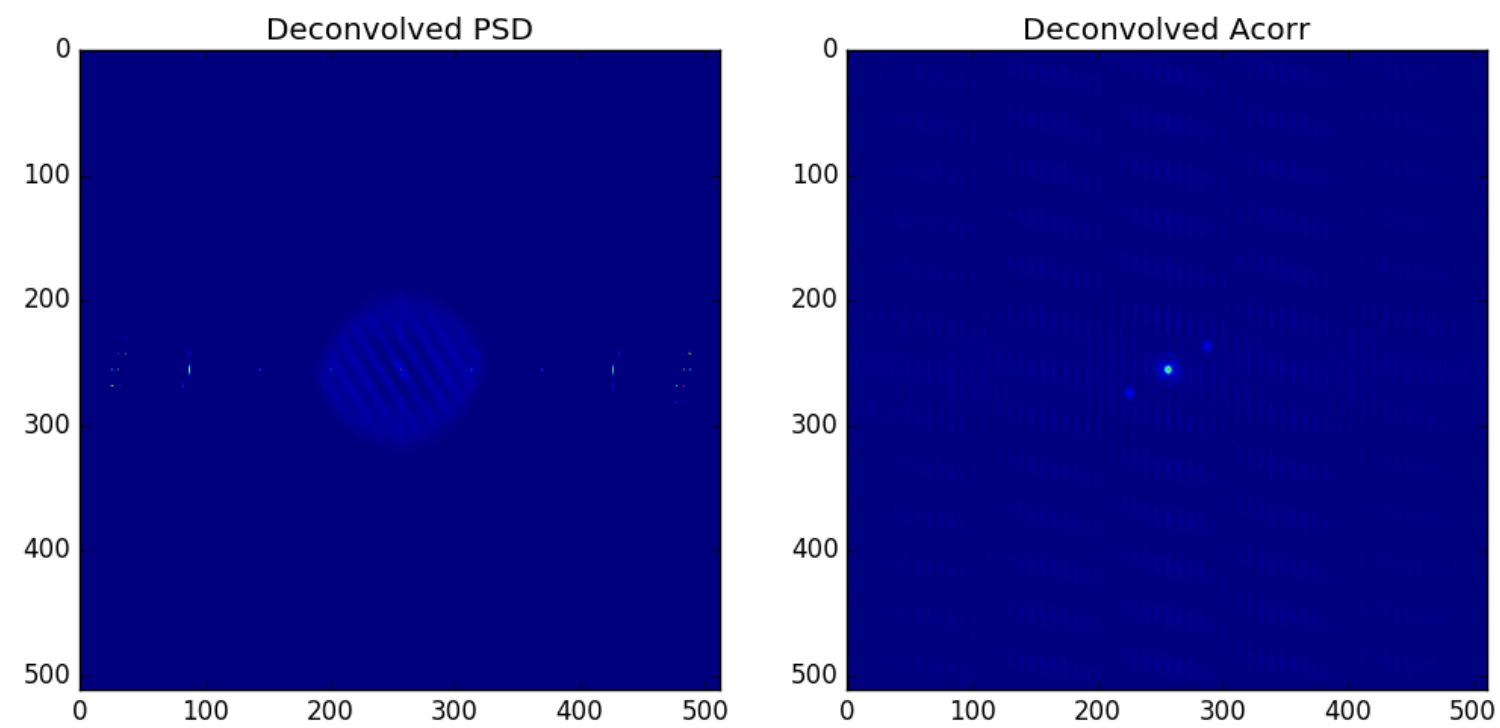

Figure 3.9: Applying simplified Wiener filter to KPNO data

To minimize this high frequency noise, knowledge of the signal to be recovered is employed. Because both the binary and reference star images are captured through the telescope, it is known that the image captured by the camera will be confined to the spatial frequency band passed by the telescope. So the $S_{f}(u, v)$ term, the PSD of the recovered signal, may be set to a lowpass function instead of a constant. This has the effect of emphasizing the low frequency information and attenuating the high frequency information, which is known to be noise. To prevent ringing in the corresponding autocorrelation, a Gaussian lowpass filter is used for $S_{f}(u, v)$ with a cutoff frequency slightly higher than the telescope's cutoff frequency. The constant $S_{n}(u, v)$ term remains a constant, and is adjusted to give 
satisfactory deconvolution. Results of this Wiener filter deconvolution are shown in Figure 3.10.
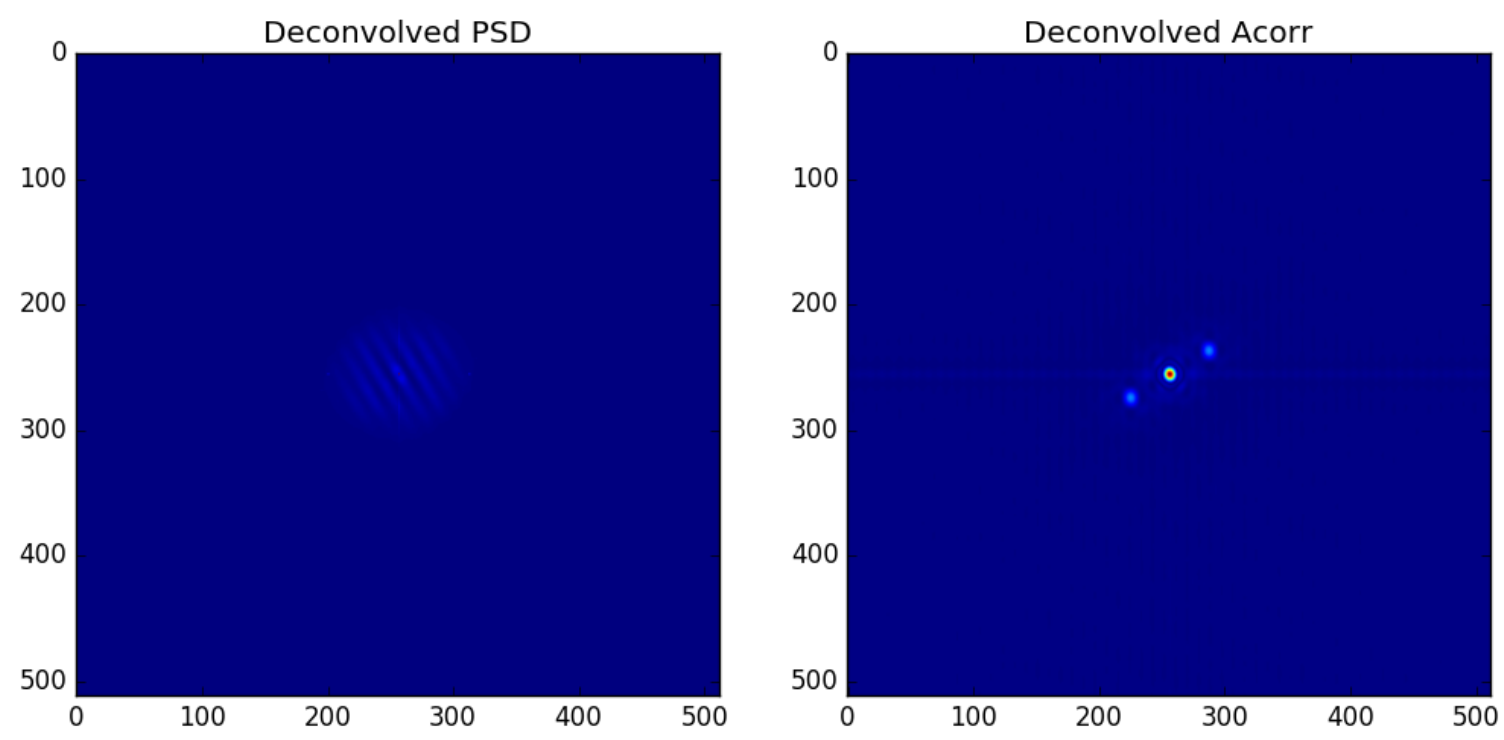

Figure 3.10: Applying second Wiener filter to KPNO data

This form of the Wiener filter allows greater control over the region to be filtered vs attenuated. It may be seen that the background noise present in 3.9 is no longer visible in 3.10. A comparison of the regions where inverse filtering is performed (regions of high SNR) with these two Wiener filtering methods is shown in Figure 3.11. Wiener filter \#1 refers to the first Wiener filter implemented with a constant SNR, Wiener filter \#2 refers to the second Wiener filter implemented with a constant $S_{n}(u, v)$ and Gaussian lowpass $S_{f}(u, v)$. Ideally, the Wiener filter would perform inverse filtering out to the diffraction limited spatial frequency of the telescope's optics (where the SNR is good), and not perform it outside this region (where the SNR is poor). Figure 3.11 shows that the Wiener filter \#2 inverse filters up to nearly the same frequencies as Wiener filter \#1, yet attenuates more high frequency noise than Wiener filter \#1. Wiener filter \#2, with its more accurate $S_{f}(u, v)$ 
model, gives a recovered autocorrelation with more visible and sharper side lobes, allowing for easier extraction of astrometric data.
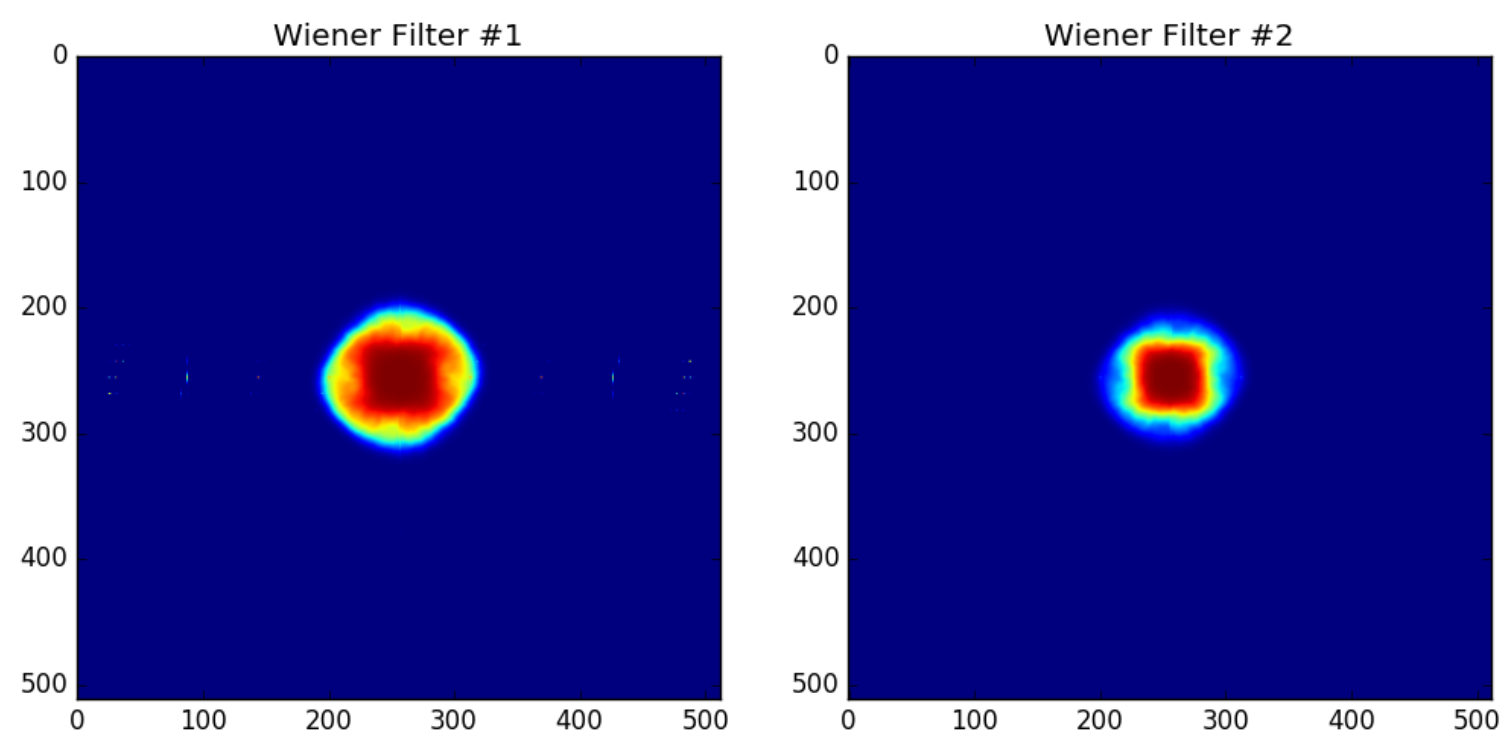

Figure 3.11: Comparison of inverse filtering regions of Wiener filters

One downside of this Wiener filtering method is that it requires different values of $S_{n}(u, v)$ for different images, depending on the relative brightness of the binary and reference stars as well as other factors. One simple alternative that works well consistently between different binary/reference star pairs is just using a gaussian lowpass filter to attenuate the high frequency noise in the inverse filtered PSD (this was the approach used in the PS3 program). This gives fairly similar results to the Wiener filter often gives, as it also preserves the low frequencies high in SNR and attenuates the high frequencies with low SNRs. This is implemented in the Labeyrie Deconvolution modules in Appendix F. This results of this deconvolution method are shown below in Figure 3.12. 

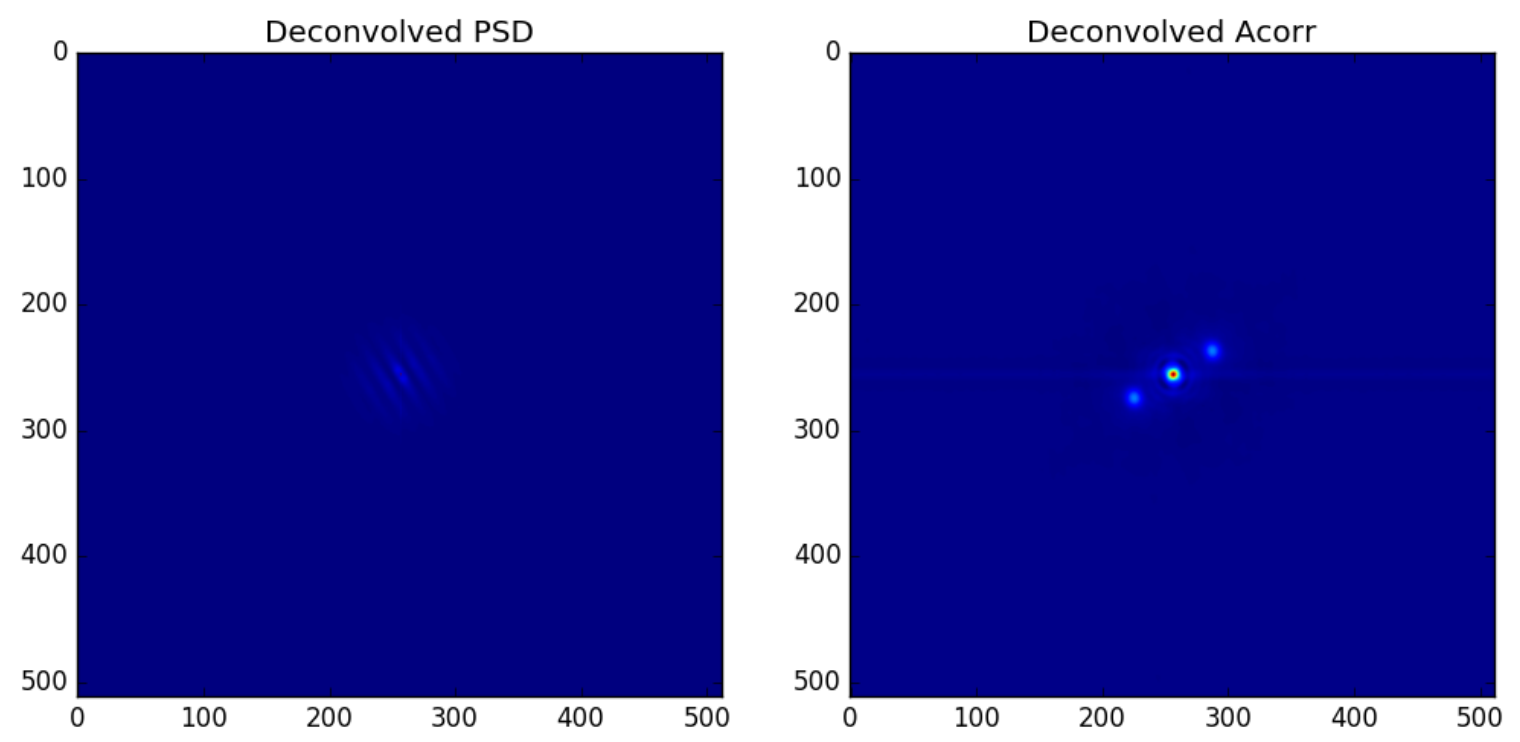

Figure 3.12: Deconvolution KPNO data with LPF/Inverse Filtering

Labeyrie's speckle interferometry process allows for the calculation of astrometric data of binary stars in the presence of atmospheric distortion. Using a reference star allows for calculating astrometric data in poor seeing conditions.

Python scripts used to generate the images in this section are found in Appendix E.

\subsection{Implementation}

To allow for automation of speckle interferometry by students and instructors at different universities, this Labeyrie deconvolution method was implemented in several Python modules. Example code was written implementing these modules to preprocess large quantities of .FITS data cubes to PSD .FITS files and creating autocorrelations from deconvolved binary/reference star PSDs. The 1000-512x512 image .FITS data cubes that have been commonly used for observations take up $1 \mathrm{~GB}$ when uncompressed. This means that from several nights of observing, data 
on the order of 500GB-1TB can be generated. This makes it difficult to transfer the data over the internet, which is especially important for remote observatories. The preprocessed PSD .FITS file from a 1GB .FITS data cube is $1.1 \mathrm{MB}$, and holds all the information needed for this Labeyrie Deconvolution process. This is a nearly $1000 x$ reduction in data size, making data transfer significantly faster. The modules written are shown in Appendix F and the scripts that use them to preprocess and deconvolve are shown in Appendix G. 


\section{IMPLEMENTATION IN AUTOMATED OBSERVATORY}

\subsection{Optimization}

The present tool used for processing speckle interferometry data by Dr. Genet's students is the "PS3" tool, written by David Rowe [31]. This is a fully featured tool for deconvolving reference from binary stars, preprocessing data, filtering data, and other functions. The basic workflow and timing for using PS3 to generate the autocorrelation of a deconvolved binary and reference star is shown below in Figure 4.1. The workflow revolves around working with large .FITS cubes of data from a past observation.

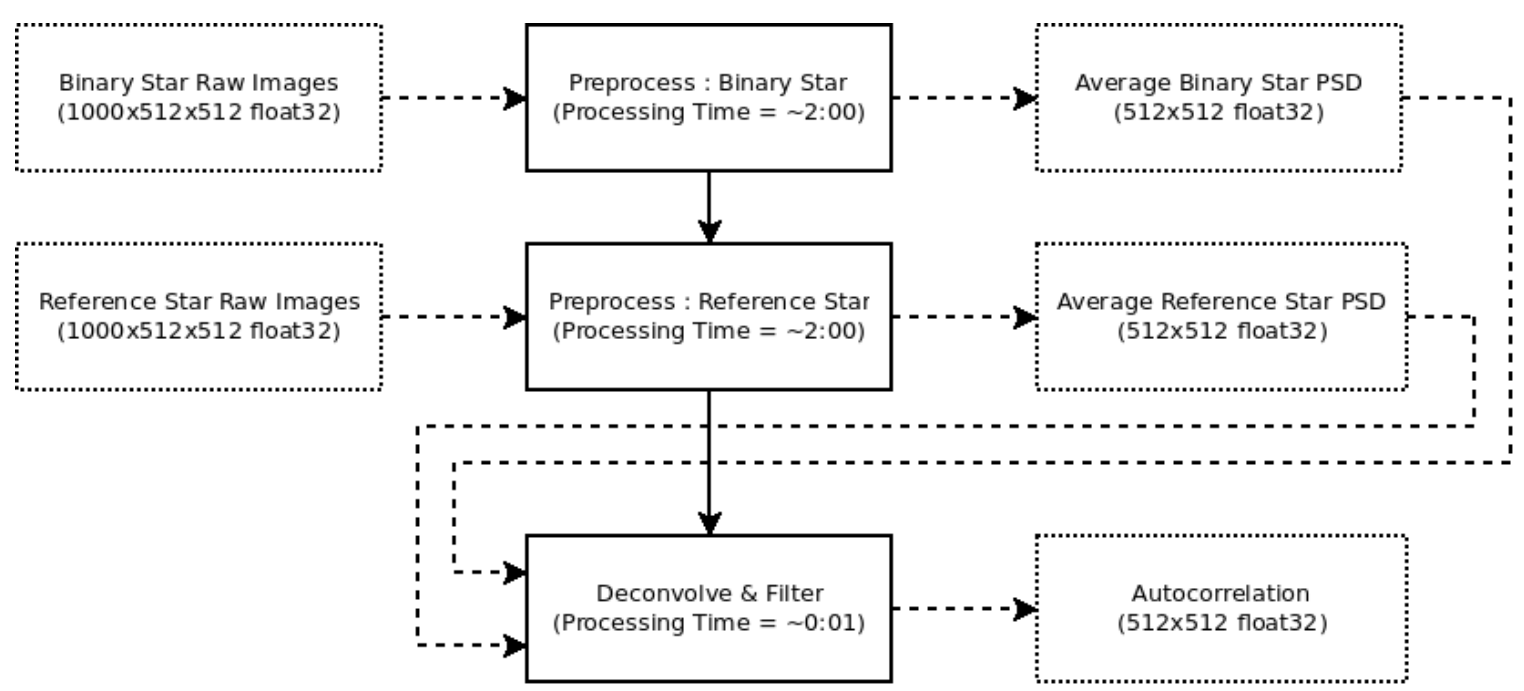

Figure 4.1: Workflow with PS3 Software

This shows that the vast majority of the time is spent in the preprocessing stage, where the PSD of each image is calculated and averaged. Common data set sizes are around 1000 images, so speeding up the calculation of the PSD could greatly increase the speed of the overall calculation. 
Various methods were used to calculate the PSD of each image in Python to find the fastest. The most basic method was working only in Python, utilizing the NumPy and SciPy FFT algorithms. In an attempt to further increase calculation speed, the PSD calculation was done in Cython, with the expectation that the static type declarations could speed up these calculations. Finally, the FFTW FFT algorithm was used in a PSD calculating C function, which was then wrapped for use in Python using the Python ctypes module. The computation time of the PSD of a $512 \times 512$ float32 image using each technique is shown below in Figure 4.2, with the code used for this benchmark shown in Appendix $\mathrm{H}$ :

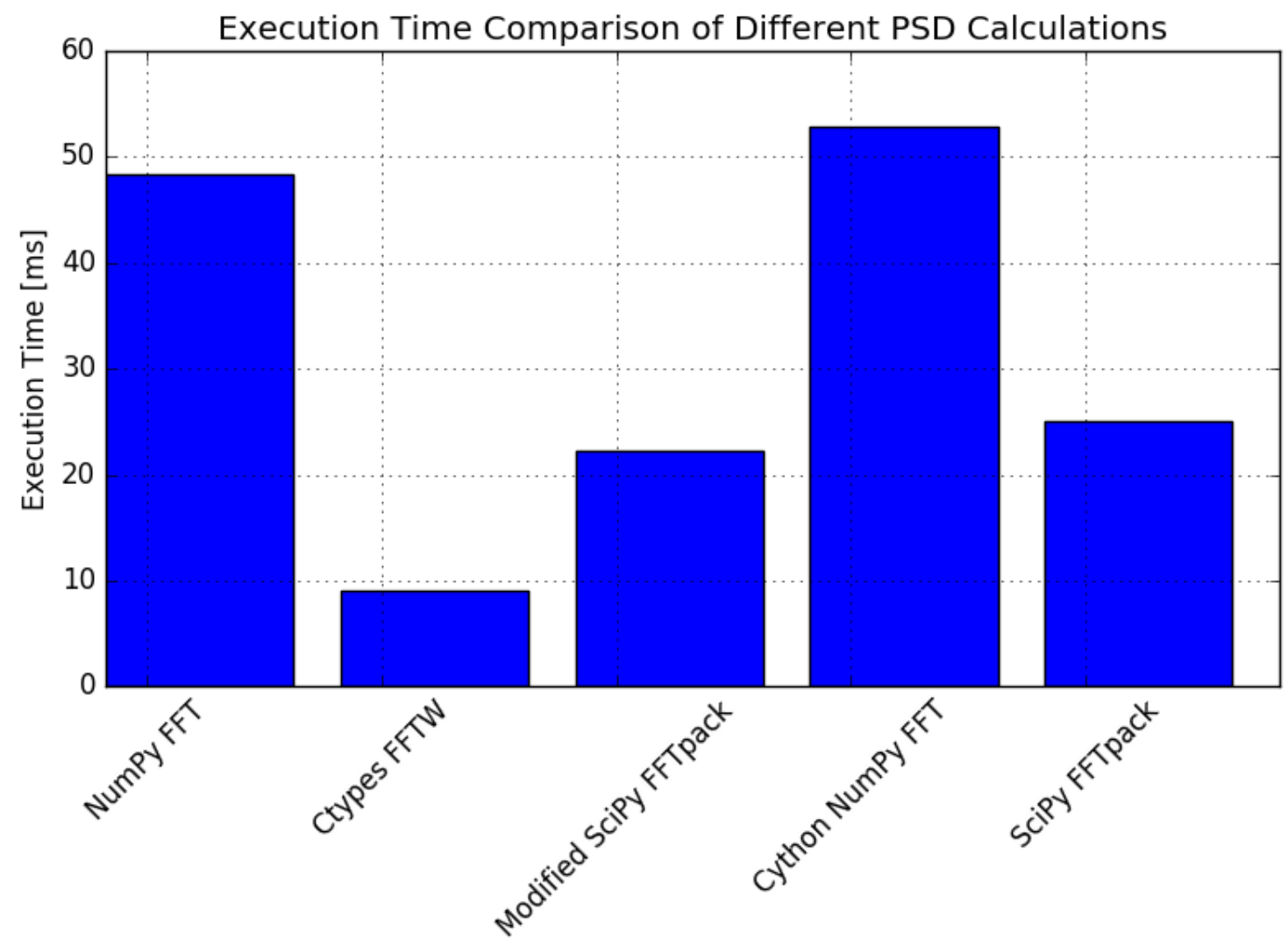

Figure 4.2: Comparison of PSD Calculation Techniques 
The wrapped C code, which utilizes the notoriously fast FFTW algorithm, was the fastest. One of the large gains from using the FFTW function is its special "R2C" function, for calculating the DFT of real data. The DFT of real data will always be symmetric and therefor contain redundant data [32]. The FFTW R2C DFT only calculates the non-redundant data, speeding up the calculation. The most visible tradeoff for this speed increase was simply development time: NumPy/SciPy solutions required practically zero extra research effort while utilizing wrapped C code required some effort to pass NumPy arrays back and forth between Python and C code. Using this fast PSD calculation inside the preprocessing script written in Python (seen in Appendix G) results in a preprocessing calculation of $10 \mathrm{sec}$ onds. Compared to the average 120 seconds required to preprocess in the PS3 program, this is a $12 x$ improvement.

While this speed increase is useful, greater gains for the user may be realized by implementing these PSD calculations earlier in the observation process.

\subsection{Implementation}

The common workflow that has been used for observing visual binary stars using speckle interferometry is shown below in Figure 4.3:

Because of the many images being taken, the observation time is a significant portion of the overall time. Presently, during the observation time the only task in progress is the capturing of photos by the camera. This means that the computer used for the observation is sitting idly, not being fully utilized. With more au- 
tomation, the observation and preprocessing time may be combined to give faster results.

Dr. Ridgely is currently designing an assembly of flip mirrors, filter wheels, cameras, and a computer to be mounted on a telescope. This assembly would allow for several different observations without changing equipment, would be suitably rigid/integrated to give repeatable results, and would only require connection of one cable each for power and communication (versus the array of communication/power cables that would be required if each part was controlled separately). In addition, software is being designed to automate all of these parts together. A large part of this software is improving the user experience of capturing speckle interferometry data. One of the major improvements to be made is the display of an autocorrelation in near real time, which would allow the observer to confirm that the target and settings were correct, preventing time-wasting errors. For this to be possible, the proposed workflow shown below in Figure 4.4 is to be implemented:

Because the wrapped C function allows for 9ms PSD calculations and the exposure and data transfer overhead time of an individual image is often in the range of $10-100 \mathrm{~ms}$, the PSD of each image may be calculated and accumulated immediately after it is received by the computer by starting a thread in parallel to the main picture taking thread. This results in a near real-time average PSD available to the user, which may be transformed into an autocorrelation whenever desired. This means that the deconvolved autocorrelation of a reference and binary star may be displayed almost immediately after both observations have been completed, as there is no more preprocessing task to be done. 
Utilizing this automated camera assembly and software should allow for fewer mistakes and less time wasted, allowing for more high-quality data to be taken during an observing run at a large telescope. 


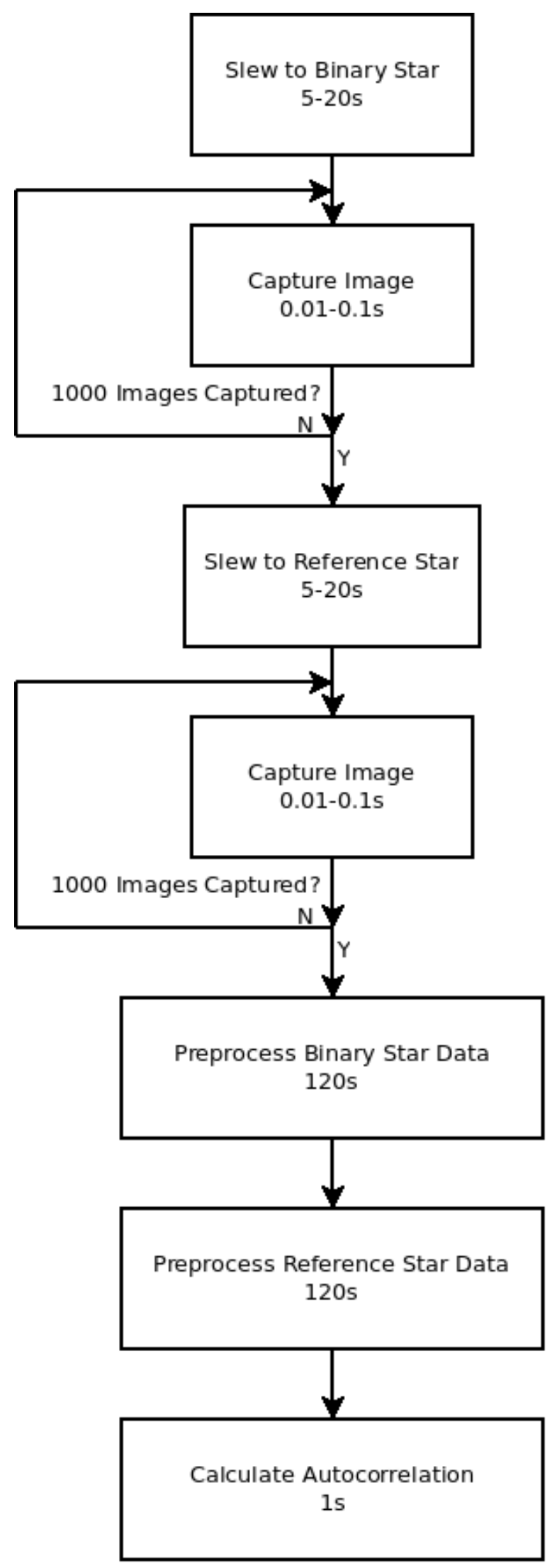

Figure 4.3: Present workflow for binary star observations 


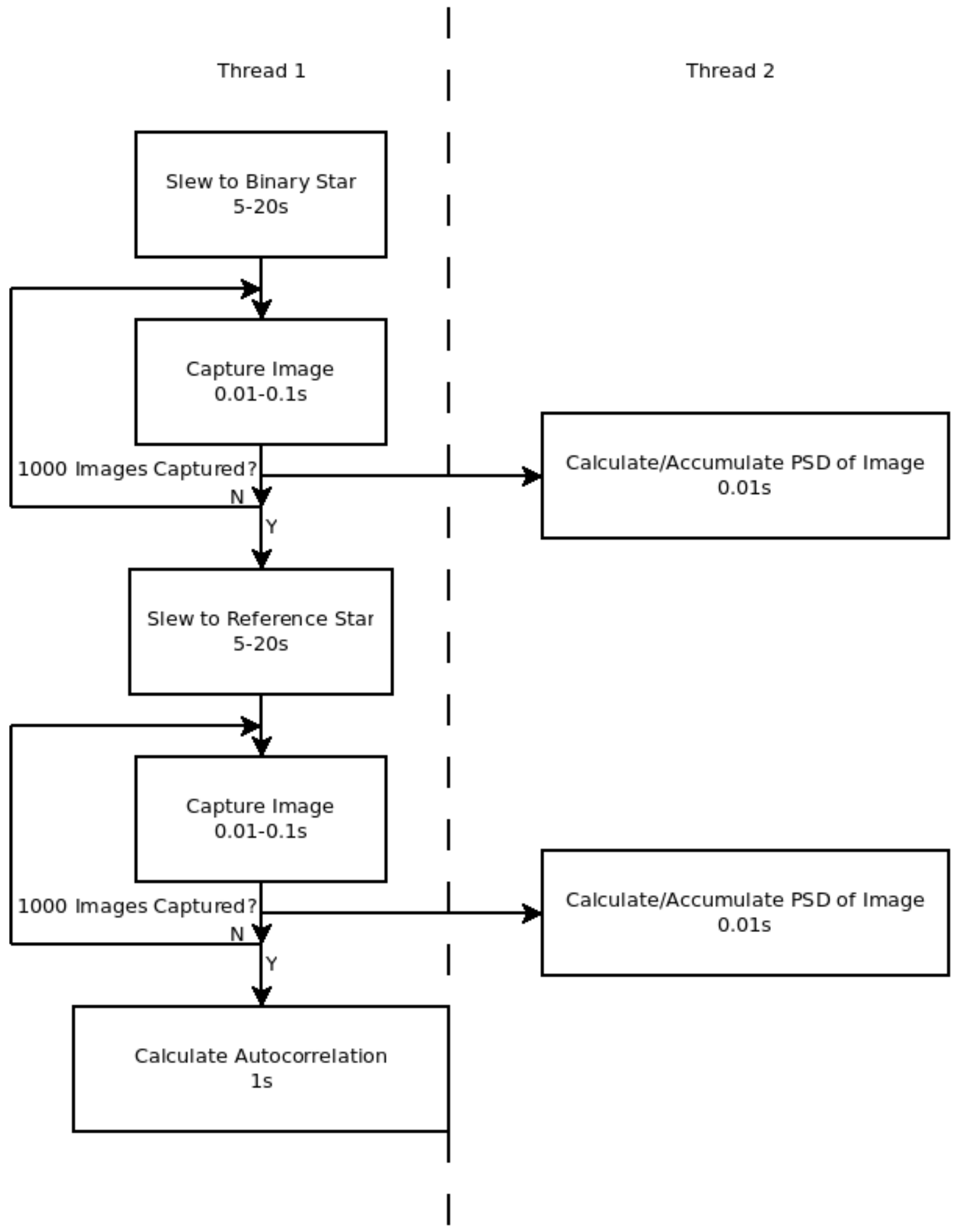

Figure 4.4: Proposed workflow for binary star observations 


\section{FUTURE WORK}

The implementation of these Python modules in automated observatories is the next step after this thesis. As previously explained, these modules will be used in John Ridgely's camera assembly to allow for near real-time viewing of PSDs of observed stars and reduce the workload of the astronomer.

The next major step in this project is the implementation of tools for doing phase recovery, which would allow for recovery of the original images with no ambiguity. Recovery of original images allows for taking photometric measurements as well. All of the phase recovery techniques are very computationally intense, and could be sped up significantly by utilizing a coprocessor, such as the Intel Xeon Phi.

All the simulations in this thesis are more qualitative than quantitative, and could be improved by students stronger in the fields of image processing and computer science. This would allow for superior simulations of real observed conditions (with a measured Fried parameter or specified noise value), rather than a more qualitative demonstration of a phenomenon. 


\section{CONCLUSIONS}

Observation of close visual binary stars is limited by both the telescope used for observation as well as the atmosphere. A simulation of the effects of telescope optics and atmospheric distortion as well as shot and additive noise was created for generating simulated binary star data. The deconvolution process originally documented by Labeyrie [27] allows for recovery of the magnitude information from speckle images of binary stars. Simulated data as well as real data taken at Kitt Peak National Observatory was successfully processed by Python modules for performing Labeyrie's deconvolution process, giving diffraction limited autocorrelations. A Wiener filter with a constant SNR was shown to be effective for deconvolving simulated data. Additional noise sources in real data warranted the use of a Wiener filter modeling the passband of the telescope's aperture to attenuate noise and recover signal data out to the diffraction limit. For processing large sets of real data with no modification to the filter between binary stars, a lowpass filtered inverse filter was found to give more consistent results than either Wiener filter. The calculation of the power spectral density was shown to be the most computationally intense part of this process, and was optimized from $25 \mathrm{~ms}$ to $9 \mathrm{~ms}$ to allow for near real time PSD calculation while observing.

All code generated during this project may be found at the following GitHub repository: https://github.com/nsmidth/CPSLO-Speckle-Reduction. 


\section{REFERENCES}

[1] Australia Telescope National Facility, "Binary stars," http://www.atnf.csiro.au/ outreach/education/senior/astrophysics/binary_intro.html, (accessed February 2, 2016).

[2] R. Sutter, "Particle and wave diffraction," http://micro.magnet.fsu.edu/primer/ java/particleorwave/diffraction/.

[3] J. Cheng, The Principles of Astronomical Telescope Design. Springer, 2009.

[4] F. Chromey, To Measure the Sky: An Introduction to Observational Astronomy. Cambridge University Press, 2010.

[5] A. MacRobert, "Beating the seeing," Sky and Telescope, August 2006.

[6] R. Genet et al., "Sparse-aperture quasi-meridian telescopes," Journal of Double Star Observations, vol. 12, no. 3, pp. 287-294, 2016.

[7] R. Giovanelli and M. Haynes, "ASTRO 2201: Binary stars," http://www.astro. cornell.edu/academics/courses/astro201/binstar.htm.

[8] L. Pedrotti, "Basic physical optics," in Fundamentals of Photonics. SPIE.

[9] G. Barbastathis, "MIT OpenCourseWare: Optics lecture 3," http: //ocw.mit.edu/courses/mechanical-engineering/2-71-optics-spring2009/video-lectures/lecture-3-focusing-imaging-and-the-paraxialapproximation/MIT2_71S09_lec03.pdf. 
[10] — - "MIT OpenCourseWare: Optics lecture 7," http://ocw.mit.edu/courses/ mechanical-engineering/2-71-optics-spring-2009/video-lectures/lecture-7basics-of-mirrors-magnifiers-and-microscopes/MIT2_71S09_lec07.pdf.

[11] R. Genet et al., "Kitt Peak speckle interferometry of close visual binary stars," Journal of Double Star Observations, vol. 11, no. 1s, 2015.

[12] Andor, "Andor Luca-R Specifications," https://www.andor.com/pdfs/ specifications/Andor_Luca-R_604_Specifications.pdf.

[13] W. Beaty, "Lasers," http://amasci.com/miscon/coherenc.html.

[14] G. Barbastathis, "MIT OpenCourseWare: Optics lecture 22," http://ocw. mit.edu/courses/mechanical-engineering/2-71-optics-spring-2009/videolectures/lecture-22-coherent-and-incoherent-imaging/MIT2_71S09_lec22. pdf.

[15] — _MIT OpenCourseWare: Optics lecture 19," http://ocw.mit.edu/courses/ mechanical-engineering/2-71-optics-spring-2009/video-lectures/lecture-19the-4f-system-binary-amplitude-pupil-masks/MIT2_71S09_lec19.pdf.

[16] J. Goodman, Introduction to Fourier Optics. McGraw Hill, 1996.

[17] C. Harding, R. Johnston, and R. Lane, "Simulation of a Kolmogorov phase screen," Waves in Random Media, vol. 2, no. 3, 1992. 
[18] R. Wilson, T. Butterley, and H. Shepard, "Characterisation and simulation of atmospheric seeing," http://www.roe.ac.uk/roe/workshop/2011/presentations/ tue-pm3-richard-wilson.pdf.

[19] D. Rowe, "ASD and speckle interferometry," http://www2.lowell. edu/workshops/speckle2014/presentations/05-0120Dave\%20Rowe\% 20ASD_and_Speckle.pdf.

[20] J. Mansell, "AN021: Analysis of Kolmogorov spectrum turbulence," http://www.activeopticalsystems.com/docs/AN021_Kolmogorov\% 20Spectrum\%20Turbulence\%20Analysis.pdf.

[21] S. Hasinoff, Photon, Poisson Noise. Springer US, 2014, pp. 608-610.

[22] C. Beenakker and C. Schonenberger, "Quantum shot noise," May 2003.

[23] R. Gonzalez and R. Woods, Digital Image Processing. Pearson, 2007.

[24] Andor, "Unravelling sensitivity, signal to noise and dynamic range," http://www. emccd.com/what_is_emccd/unraveling_sensitivity/Signal_to_Noise_in_CCDs/.

[25] Quantum Scientific Imaging, "Understanding ccd read noise," http:// qsimaging.com/ccd_noise.html.

[26] A. Glindemann, Principles of Stellar Interferometry. Springer, 2011.

[27] A. Labeyrie, "Attainment of diffraction limited resolution in large telescopes by Fourier analysing speckle patterns in star images," Astronomy and Astrophysics, vol. 6, p. 85, May 1970. 
[28] W. Beavers et al., "Speckle imaging through the atmosphere," The Lincoln Laboratory Journal, vol. 2, no. 2, 1989.

[29] W. Pratt, Digital Image Processing: PIKS Scientific Inside. WileyInterscience, 2007.

[30] T. Tyson and A. Bradshaw, "UC Davis Physics 123: Shot noise," http://123. physics.ucdavis.edu/shot_files/ShotNoise.pdf.

[31] D. Rowe and R. Genet, "User's guide to PS3 speckle interferometry reduction program," Journal of Double Star Observations, vol. 11, no. 1s, September 2015.

[32] FFTW, "Multi-dimensional transforms," http://www.fftw.org/doc/ Multi_002ddimensional-Transforms.html\#Multi_002ddimensional-Transforms. 


\section{APPENDICES}

A. Prime Focus Geometric Optics

Simulation Simulation of parabolic lens focusing light of a plane wave

\section{Kitt Peak 2.1m Telescope}

From http://www-kpno.kpno.noao.edu/kpno-misc/2m_params.html, this telescope has the following specs:

- Diameter $=2.133 \mathrm{~m}=2133 \mathrm{~mm}$

- Radius of curvature $=11168.4 \mathrm{~mm}$

- Effective Focal Length $=\mathrm{R} / 2=5584 \mathrm{~mm}$

- Focal Ratio $=5584 / 2133=\mathrm{f} / 2.63$

\section{sim_prime_focus.py}

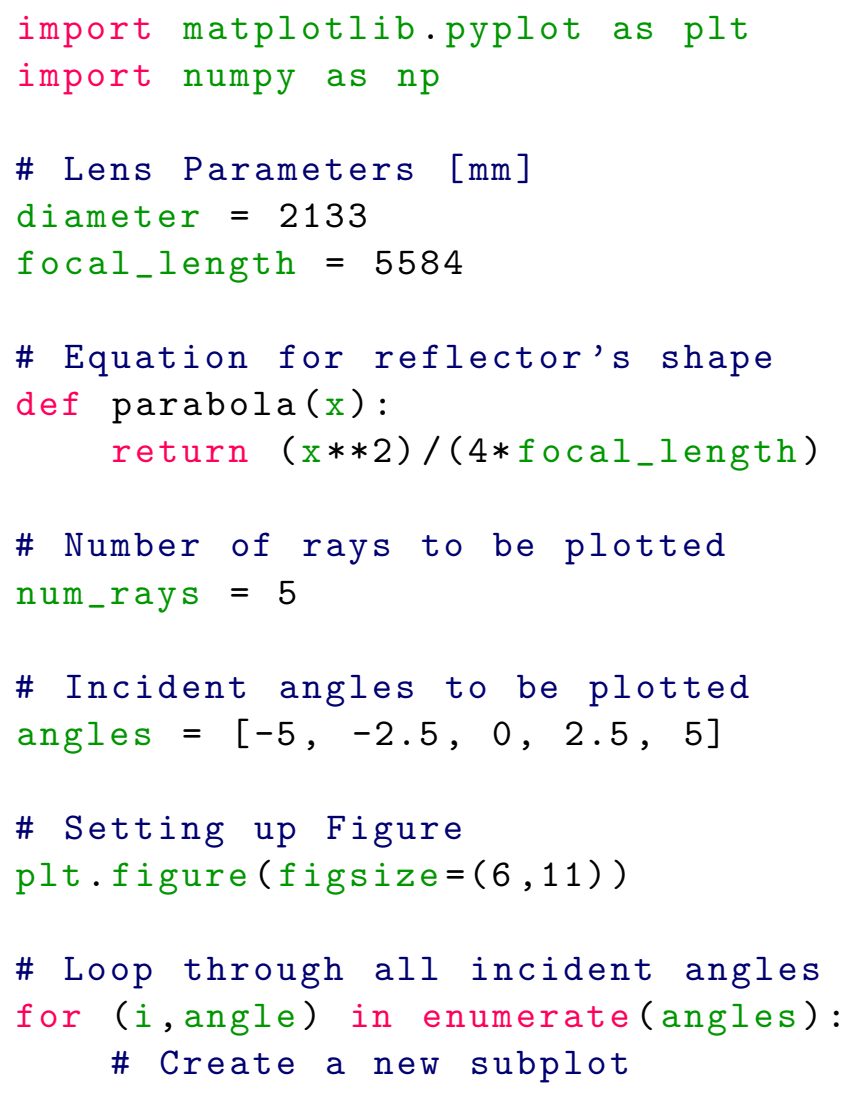




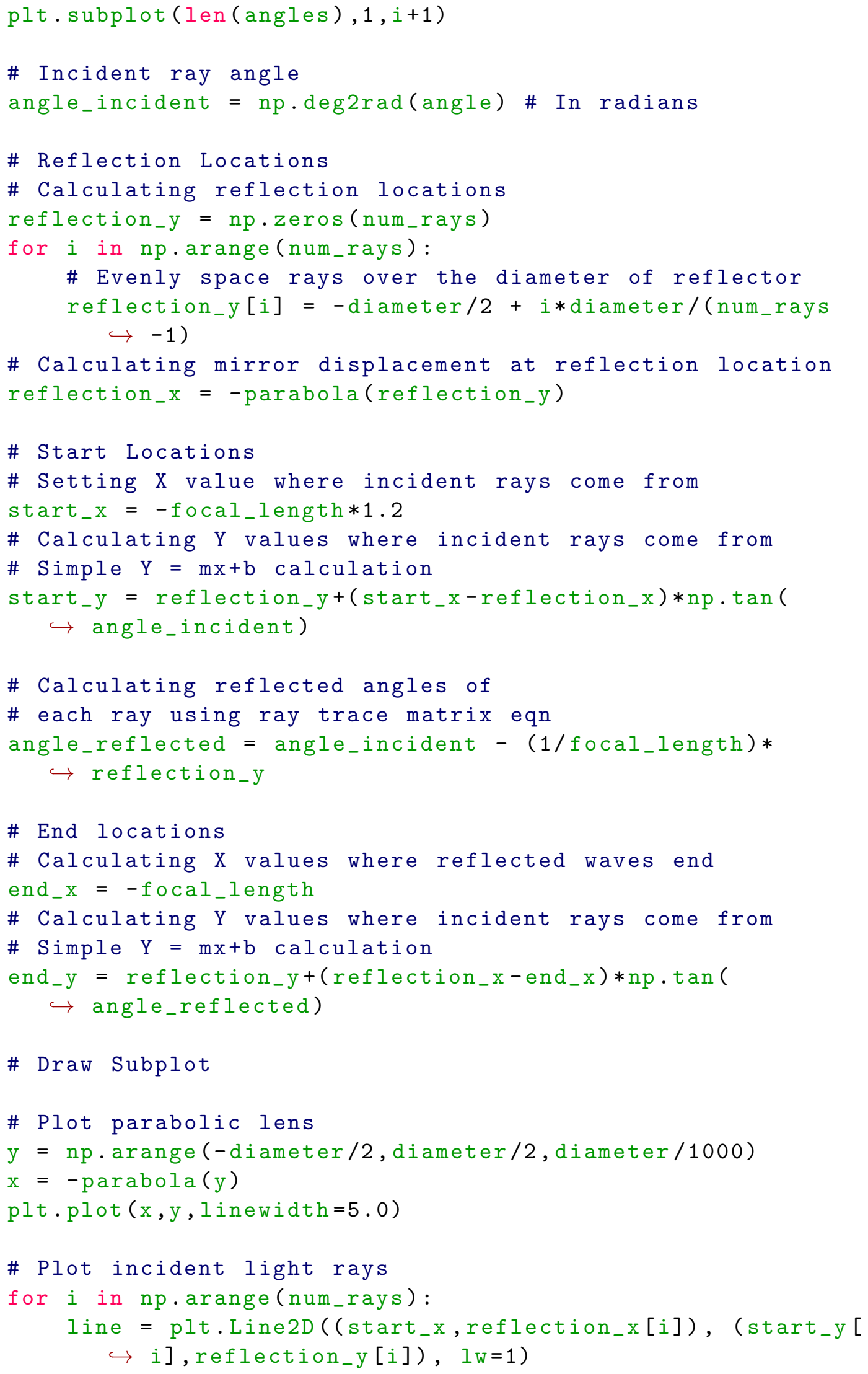




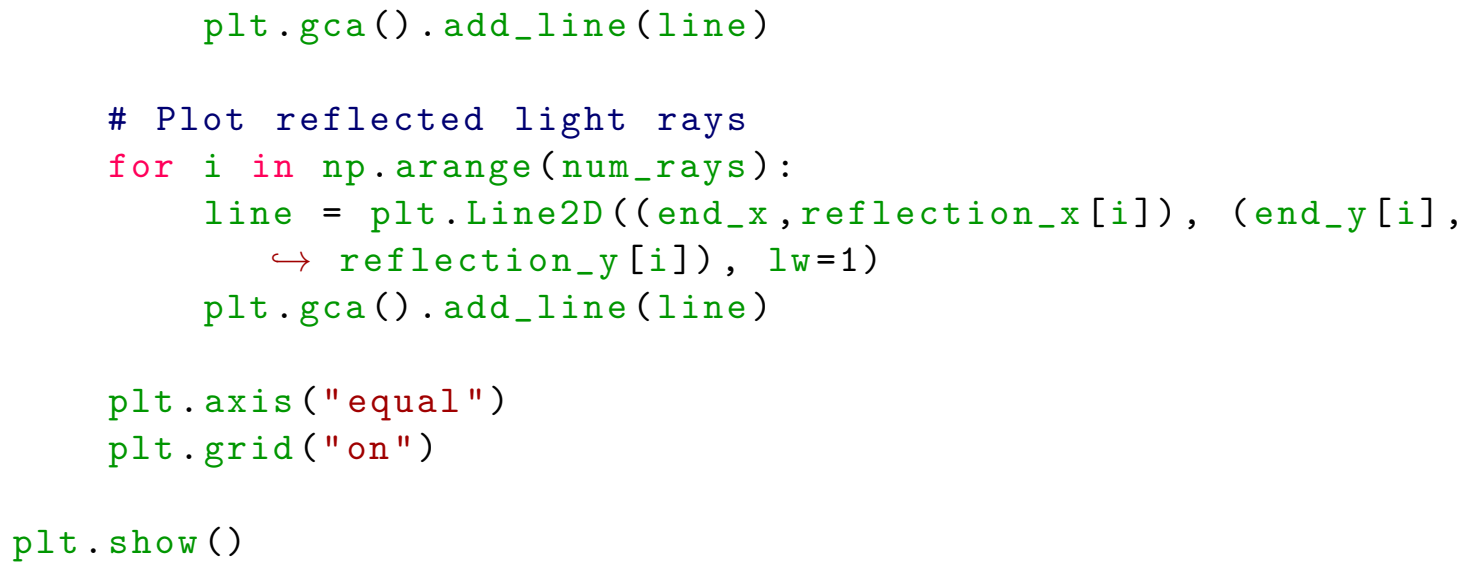


B. Prime Focus Diffraction Limit Simulation

Simulating the output of a prime focus telescope given an input image.

\section{Kitt Peak 2.1m Telescope}

From http://www-kpno.kpno .noao. edu/kpno-misc/2m_params.html, this telescope has the following specs:

- Diameter $=2133.0 \mathrm{~mm}$

- Radius of curvature $=11168.4 \mathrm{~mm}$

- Effective Focal Length $=\mathrm{R} / 2=5584 \mathrm{~mm}$

- Focal Ratio $=5584 / 2133=\mathrm{f} / 2.63$

From http://www.jdso.org/volume11/number1s/Genet_234_244.pdf, telescope was set up differently for speckle observation. Adding secondary mirror changes focal length :

- Primary Diameter $=2.1 \mathrm{~m}=2133.0 \mathrm{~mm}$

- Focal Ratio $=f / 7.6$

- Focal Length $=2133.0 \mathrm{~mm}^{\star} 7.6=16211 \mathrm{~mm}$

- 8x Barlow

- Focal Ratio $=\mathrm{f} /\left(7.6^{\star} 8\right)=\mathrm{f} / 60.8$

- Focal Length $=16211 \mathrm{~mm}^{\star} 8=129.69 \mathrm{~m}$

\section{Andor Luca-R}

From https://www.andor.com/pdfs/specifications/Andor_Luca-R_604_Specifications . $\operatorname{pdf}$

- Sensor Size $=1004(\mathrm{H}) \times 1002(\mathrm{~V})$ Pixels $=8 \mathrm{~mm}$ X 8mm 
- Pixel Size $=8 u m \times 8 u m$

- Only center $512 \times 512$ pixels used

\section{Calcs:}

Input image is in units of angles, represents the full input field of view

- This is re-sampled to image it on a pixel scale

- image scale $=\frac{206265}{\text { focallength }}=\frac{206265 \text { arcsec }}{129.69 * 10^{3}}=1.59 \frac{\text { arcsec }}{\mathrm{mm}}$

- pixel scale $=1.59 \frac{a s}{m m} * 8 * 10^{-3} \frac{\mathrm{mm}}{\text { pixel }}=12.72 \frac{\text { marcsec }}{\text { pixel }}$

- If sky input is in units of pixel scale [ex: 12.72 marcsec], then no re-sampling required

Aperture is in length units [m]

- Need to calculate the "effective" size of the aperture, taking into account effects of lens focal length and light wavelength:

- Effective Aperture Diameter $=\frac{(\text { Aperture Diameter })}{(\lambda)(\text { Focal Length })}$

- $D=\frac{(2.133 m)}{\left(0.8 * 10^{-6} m\right)(129.69 m)}=20599 \frac{1}{m}$

- This is the radius of a circle that gives the first null of the corresponding airy disc to be at $r=\frac{1.22}{D}=\frac{1.22}{20599 \frac{1}{m}}=59.34 * 10^{-6} \mathrm{~m}$

- In pixels, this first null location corresponds to $\frac{59.34 * 10^{-6} m}{8 * 10^{-6}}=7.42$ pixels

To simulate the imaging sensor, we need the sample units of the FFT of our aperture image to be the size of a pixel. For Andora Luca-R, pixel is 8um. Each sample of a $2 \mathrm{D}$ FFT represents $2 / L$ ( $L=$ total sample size of input image). So we want total spatial sampling range $=\frac{1}{L}=8 * 10^{-6}->L=\frac{1}{8 * 10^{-6}}=125 * 10^{3}$

- If this is sampled with 512 pixels, each pixel represents $\frac{125 * 10^{-3}}{512}=244.14 \frac{1}{m}$. So 
the diameter of the aperture $=D_{s}=\frac{20599}{244.14}=84$ pixels

- The FFT of this is calculated, PSF is calculated as square of magnitude

- Sanity check: first null occurs at 7 pixels away from center (256)

- Input and aperture PSF are convolved

\section{sim_diffraction_limit.py}

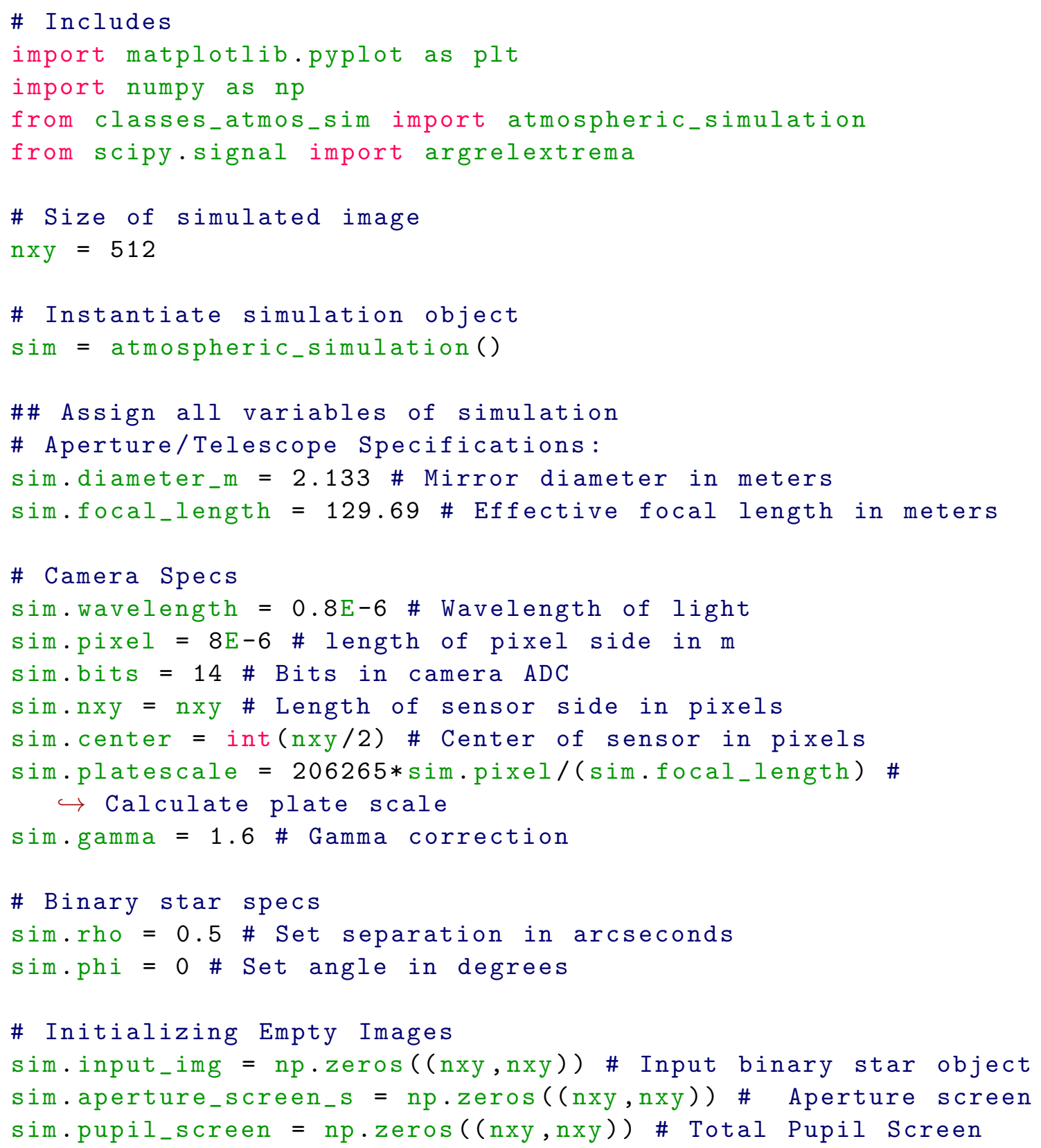




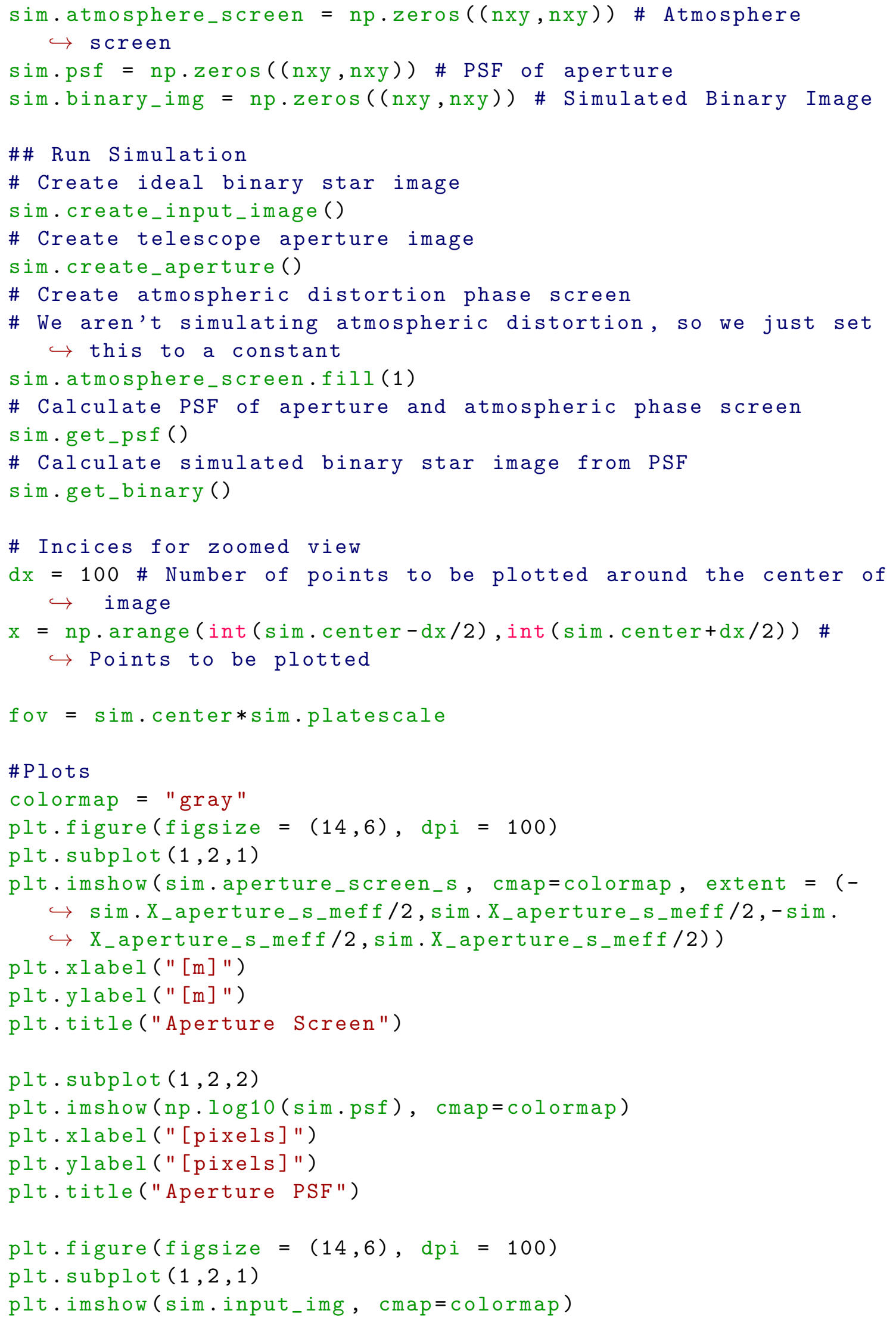




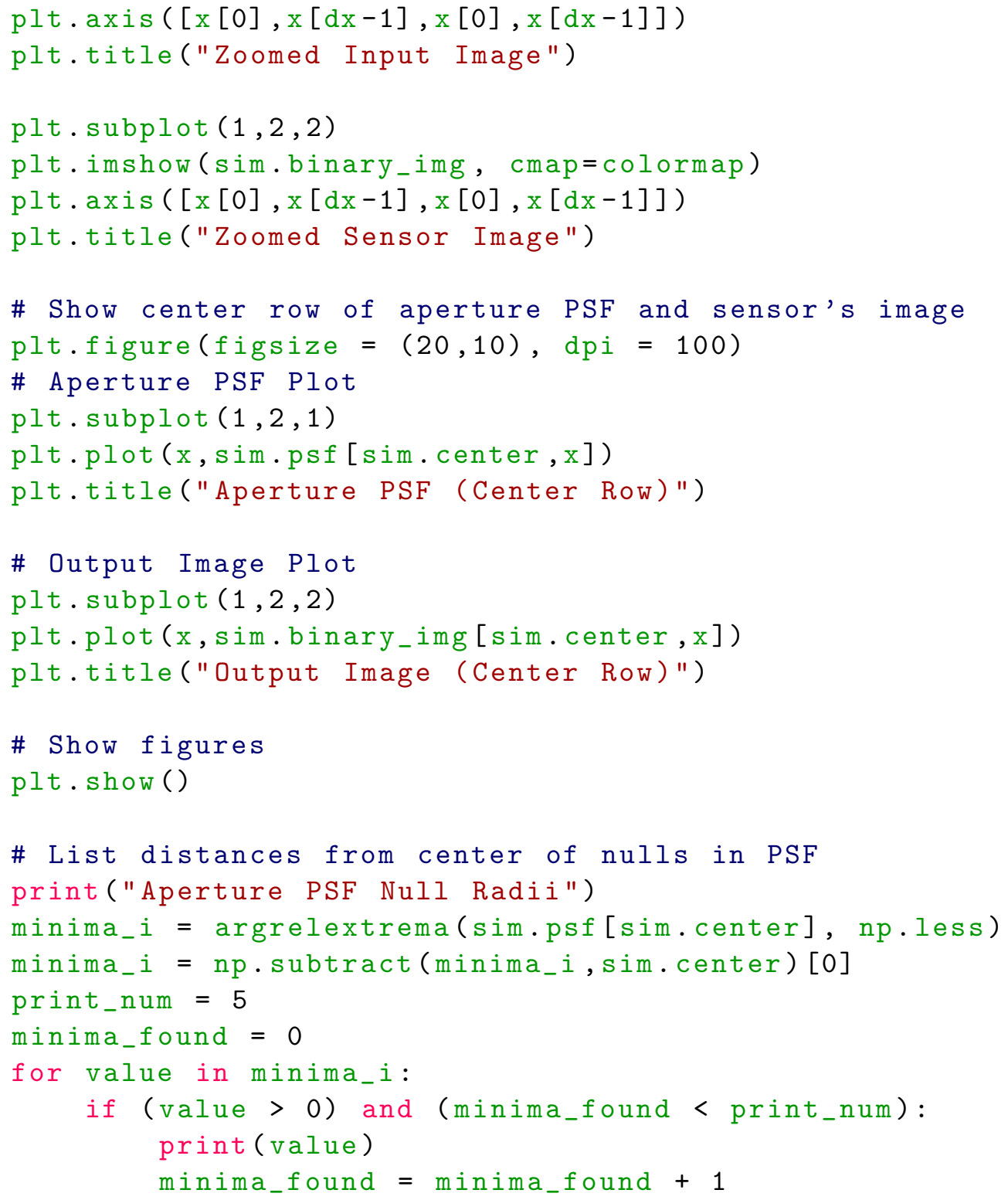

Simulation uses Atmospheric Simulation Class found in Appendix D 
C. Atmospheric Distortion Simulation

- Atmospheric distortion simulated as a phase component of the pupil plane screen

- Phase component image of pupil screen has the following PSD:

$$
\begin{gathered}
P S D_{\text {phase_image }}=\alpha * k^{-11 / 3} \\
\text { phase }_{\text {phase_image }}=\text { gaussian with mean }=0, \text { variance }=1
\end{gathered}
$$

These are combined to give the frequency domain representation of the phase image as

$$
\text { phase_screen_F }=\sqrt{P S D_{\text {phase_image }}} * \exp \left(j * \text { phase }_{\text {phase_image }}\right)
$$

- Take iFFT of frequency domain representation of phase to get phase values

$$
\text { phase_screen }=i F F T(\text { phase_screen_F })
$$

- Create pupil screen as:

$$
\text { pupil_screen }=\mid \text { aperture_mask } \mid * \exp (j * \text { phase_screen })
$$

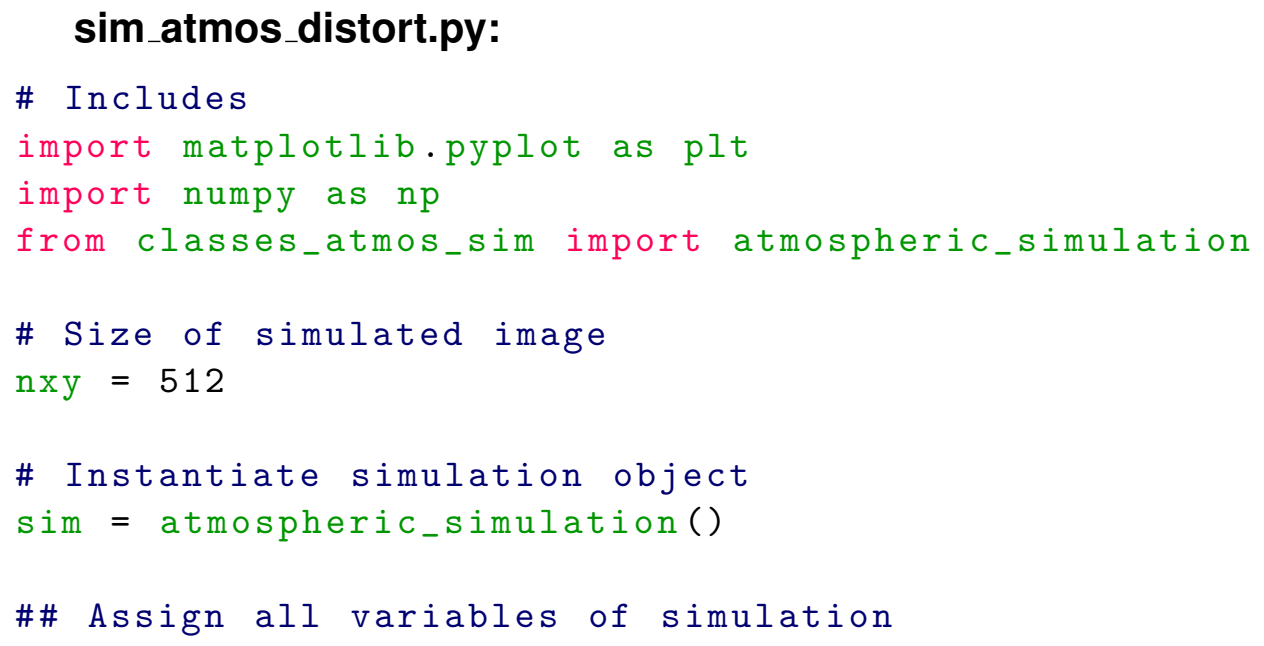




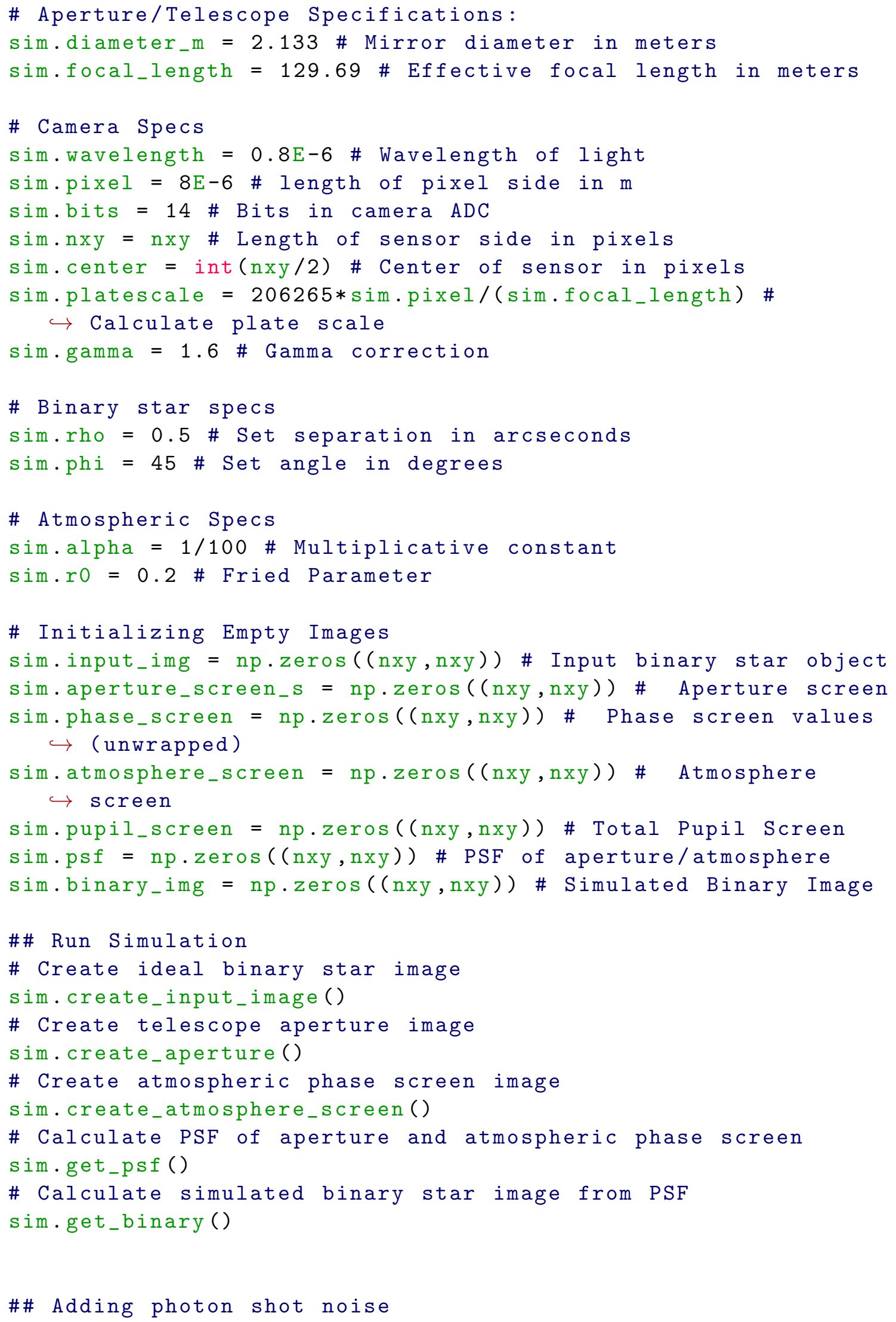




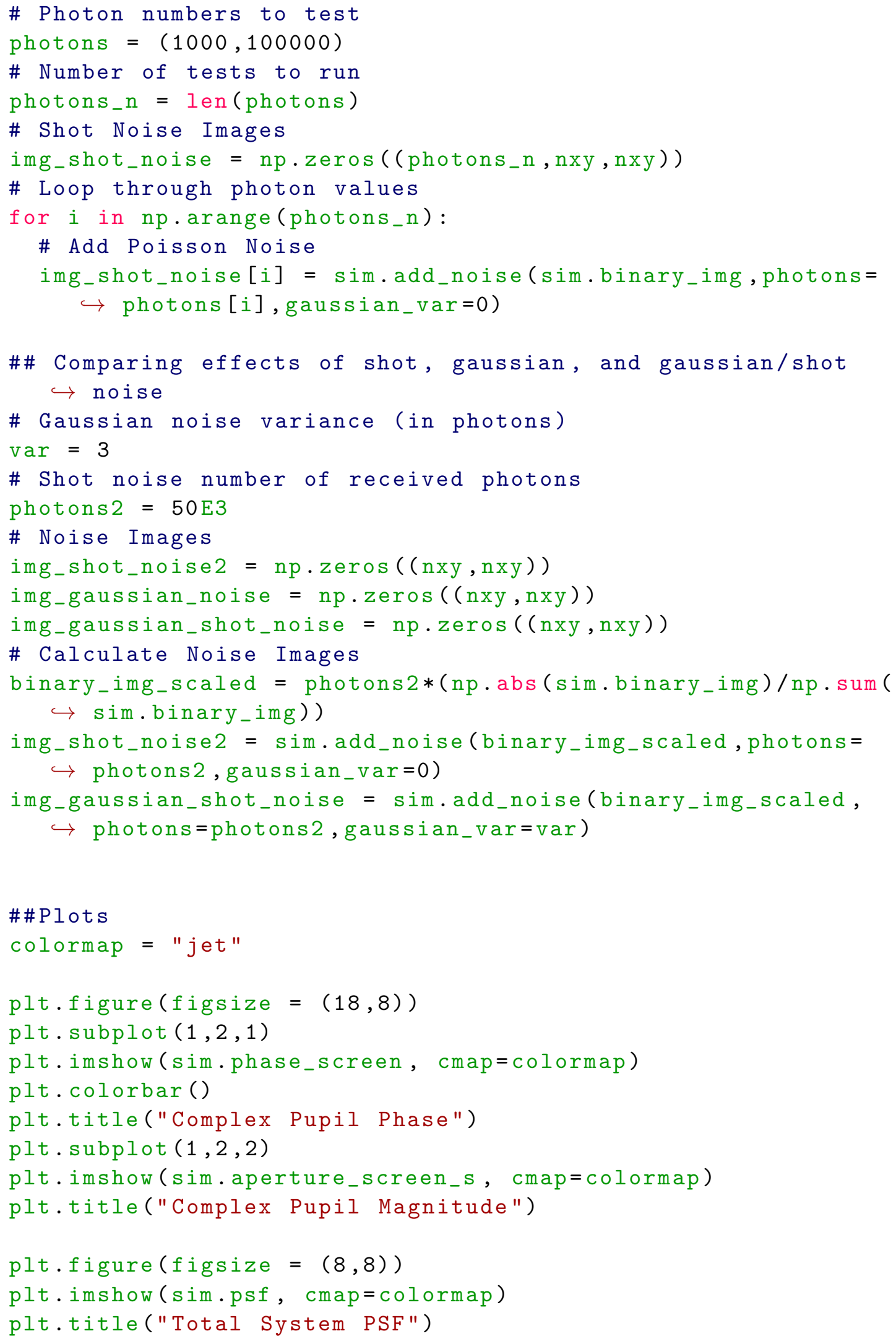




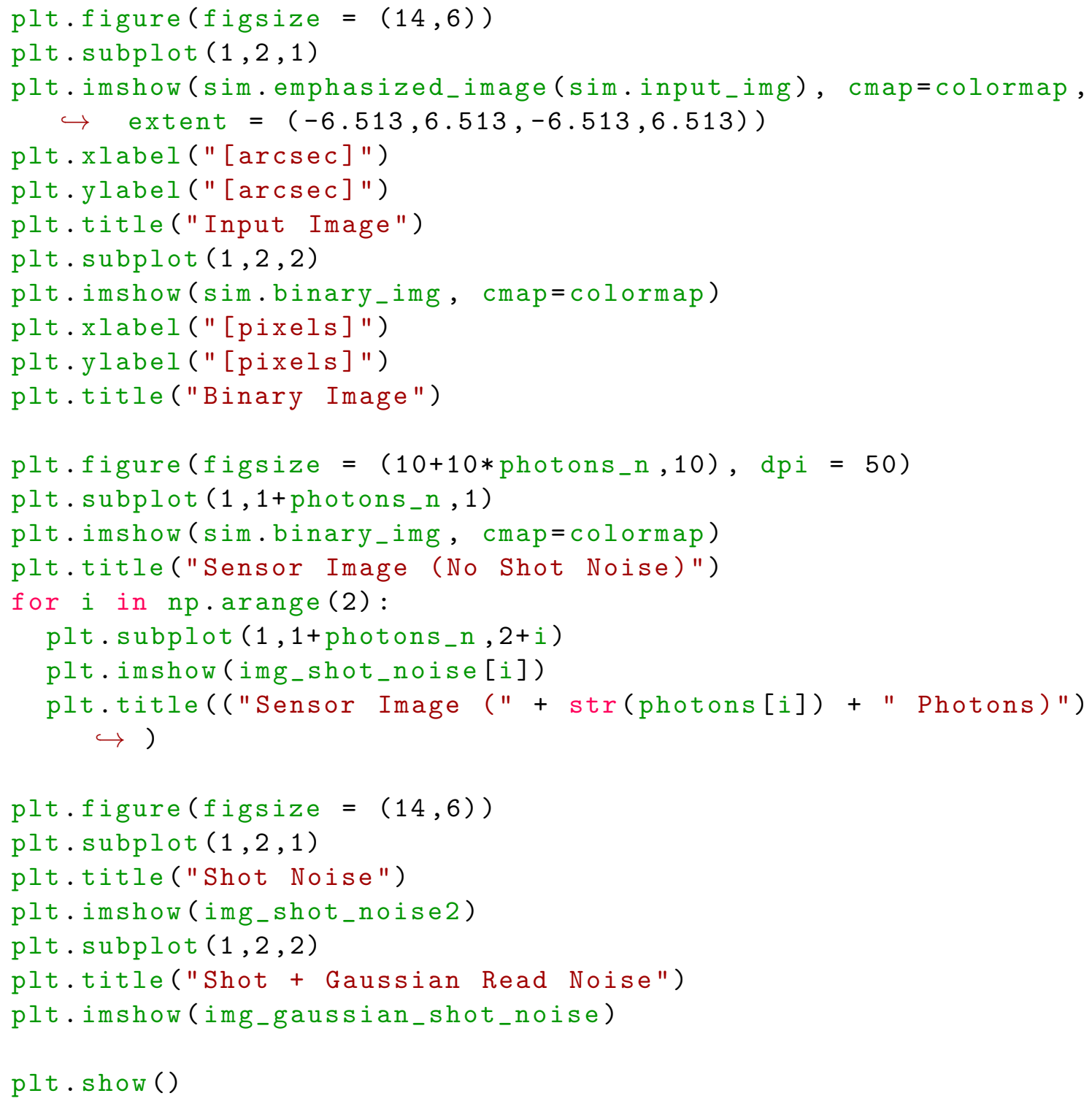

\section{Simulation uses Atmospheric Simulation Class found in Appendix D.}




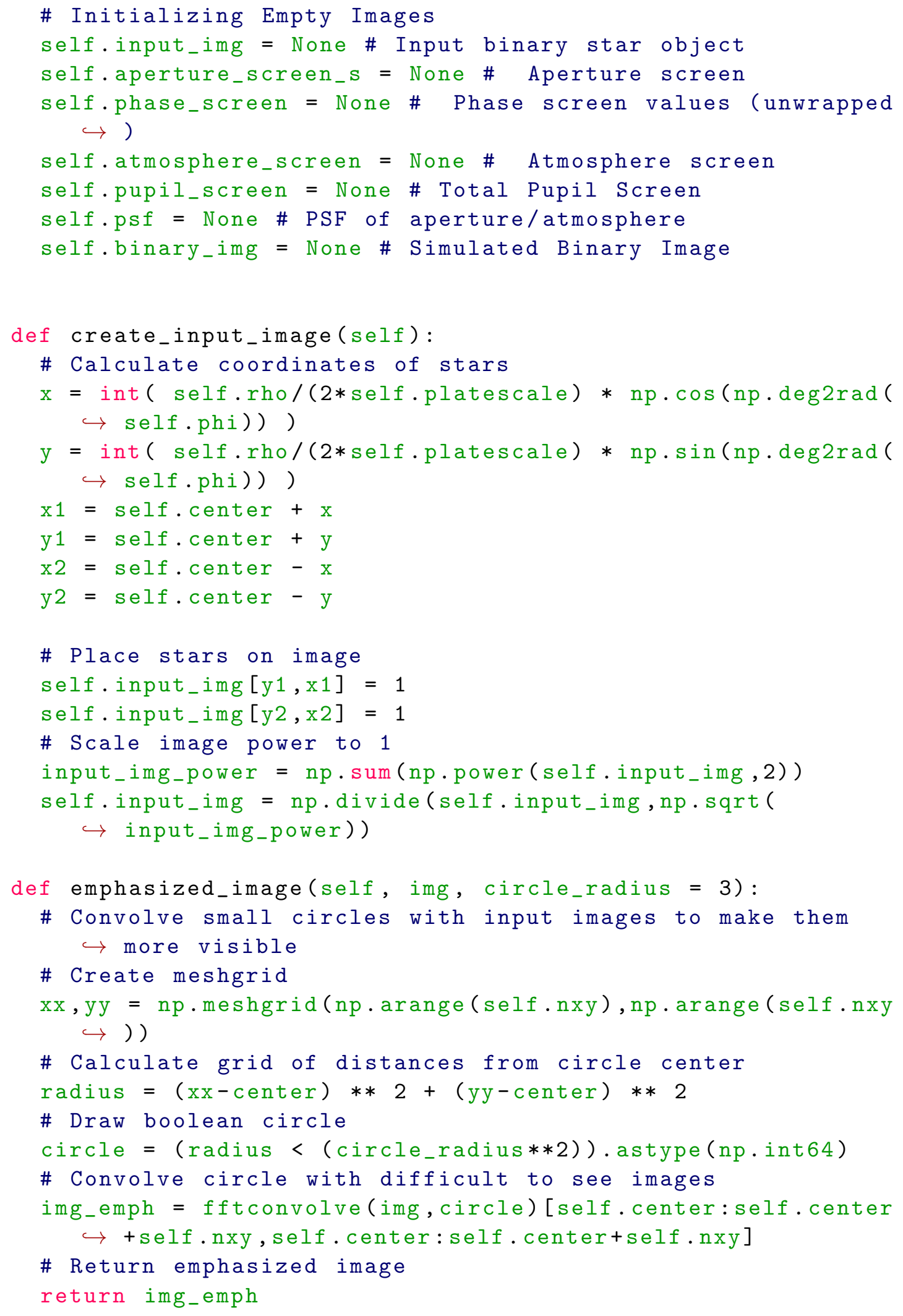




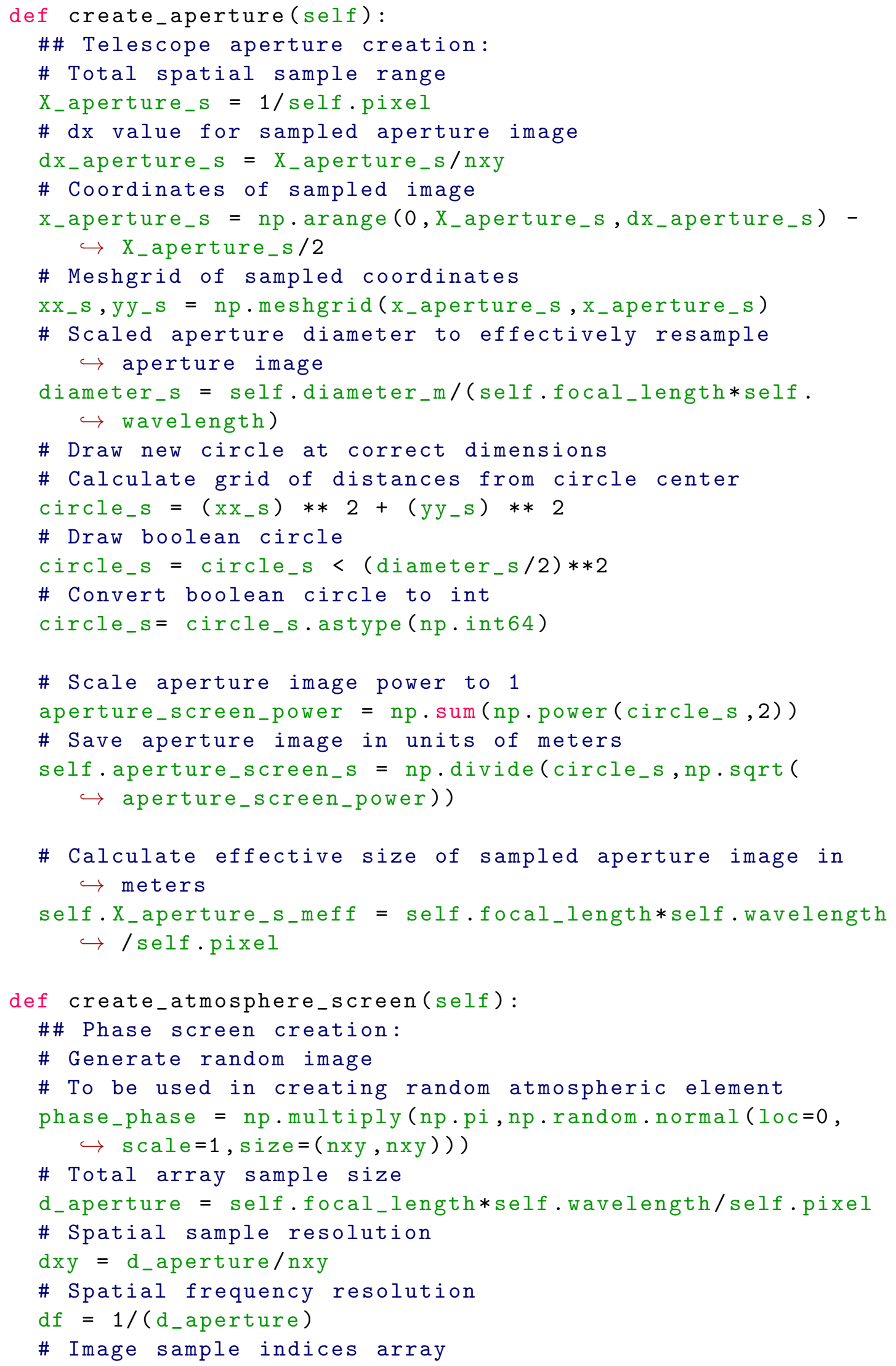




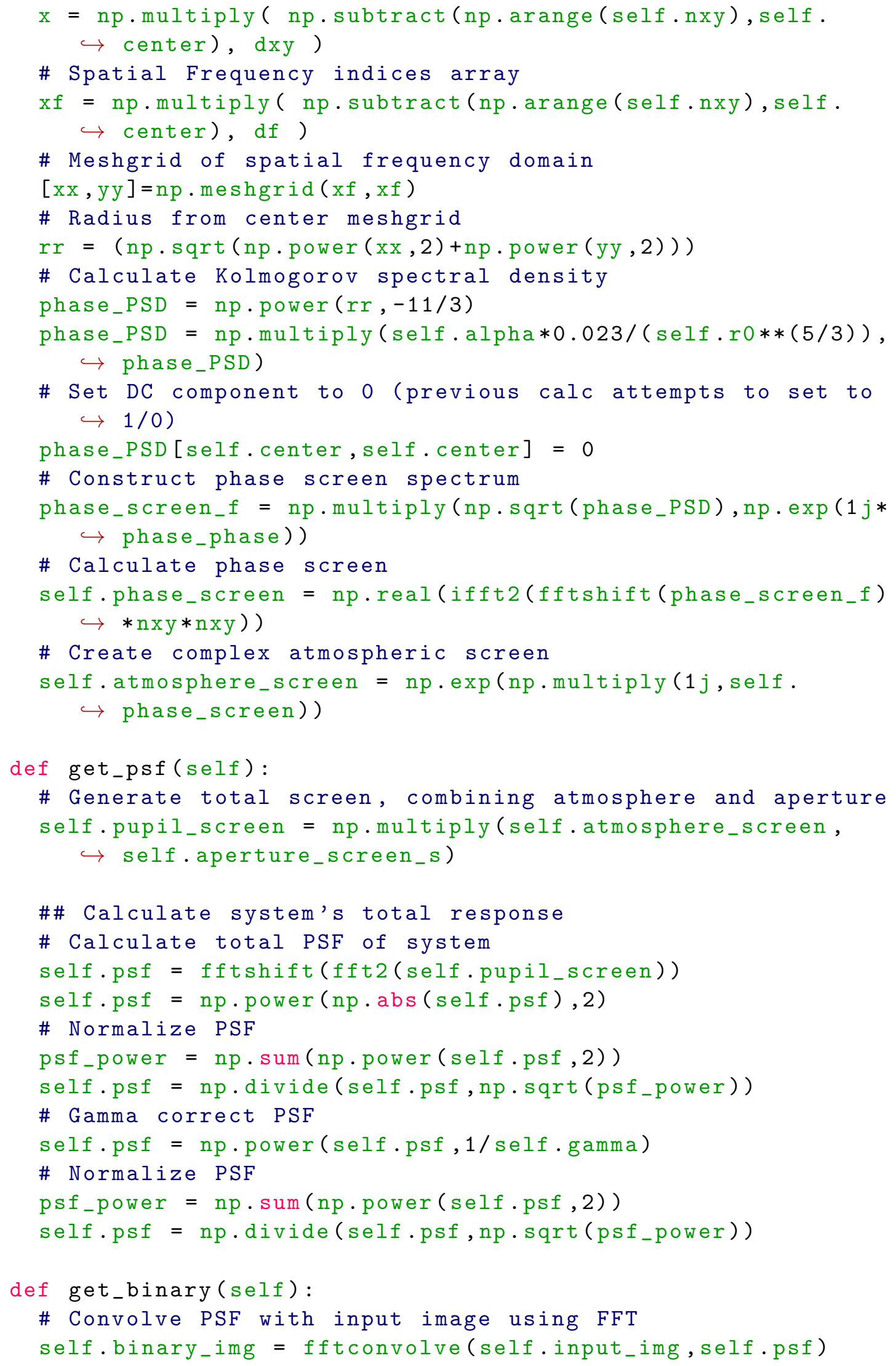




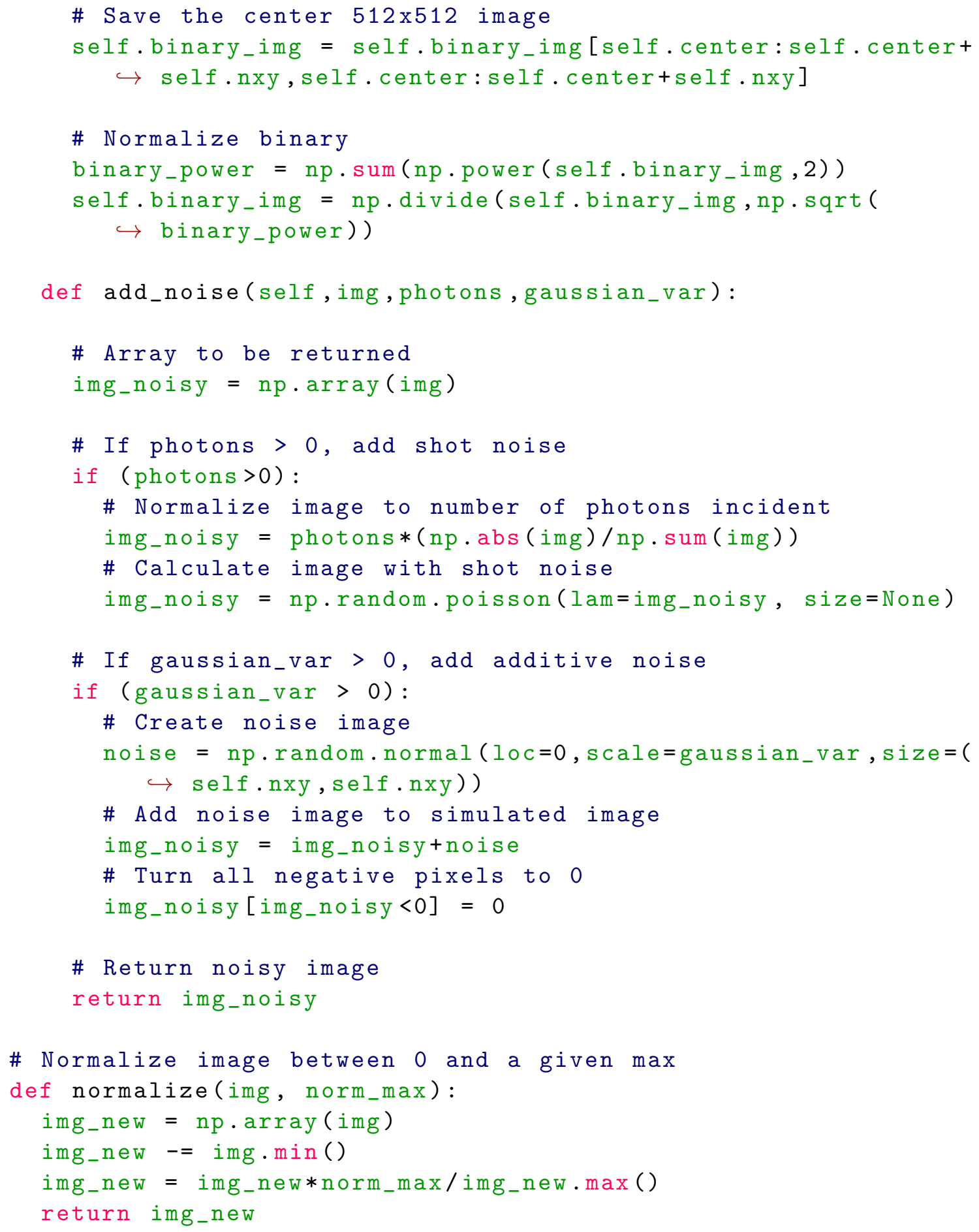




\section{E. Labeyrie Deconvolution Simulations}

Simulation uses Atmospheric Simulation Class found in appendix D.

Simulation of noiseless Labeyrie deconvolution:

\section{sim_labeyrie_deconv.py:}

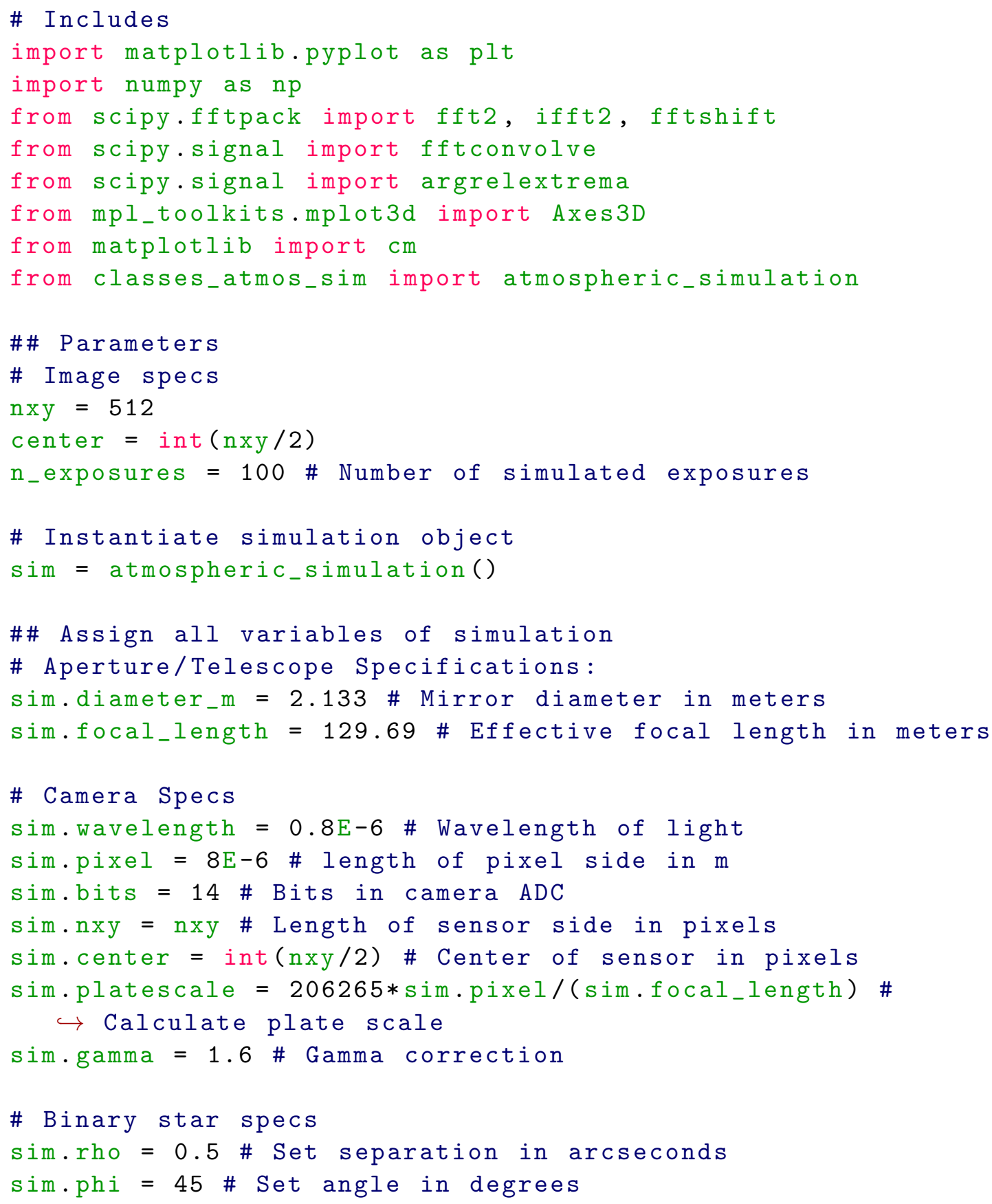




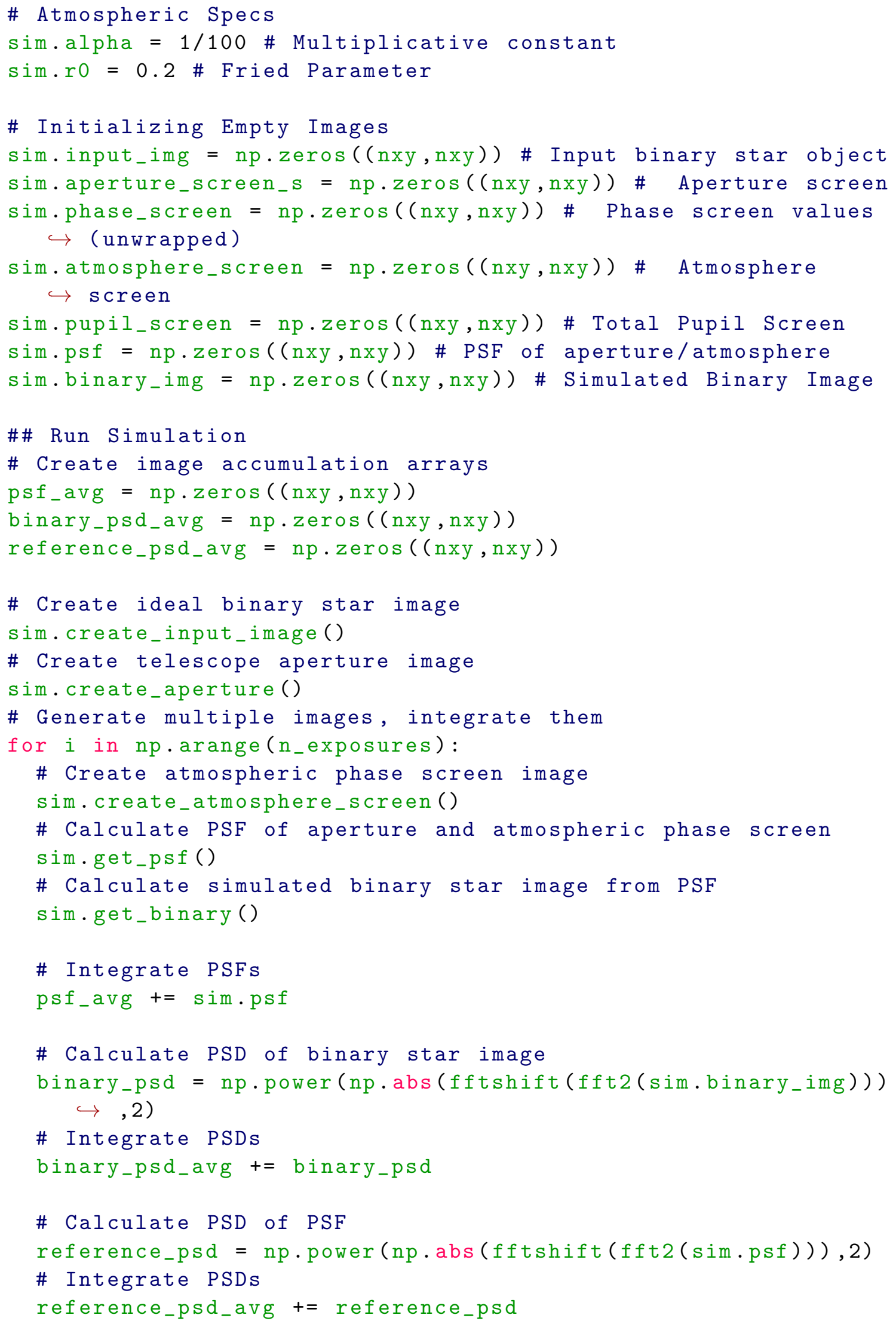




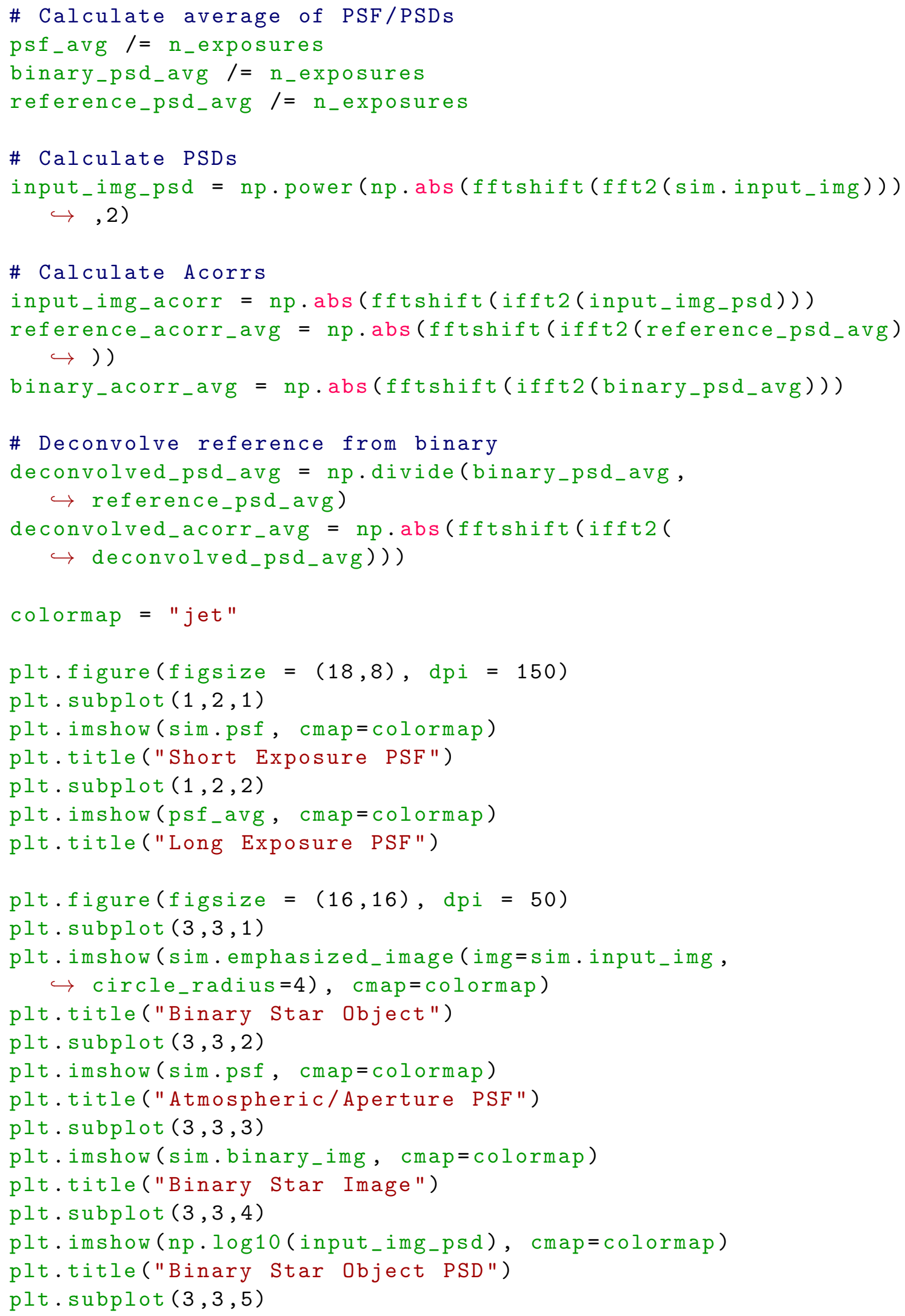




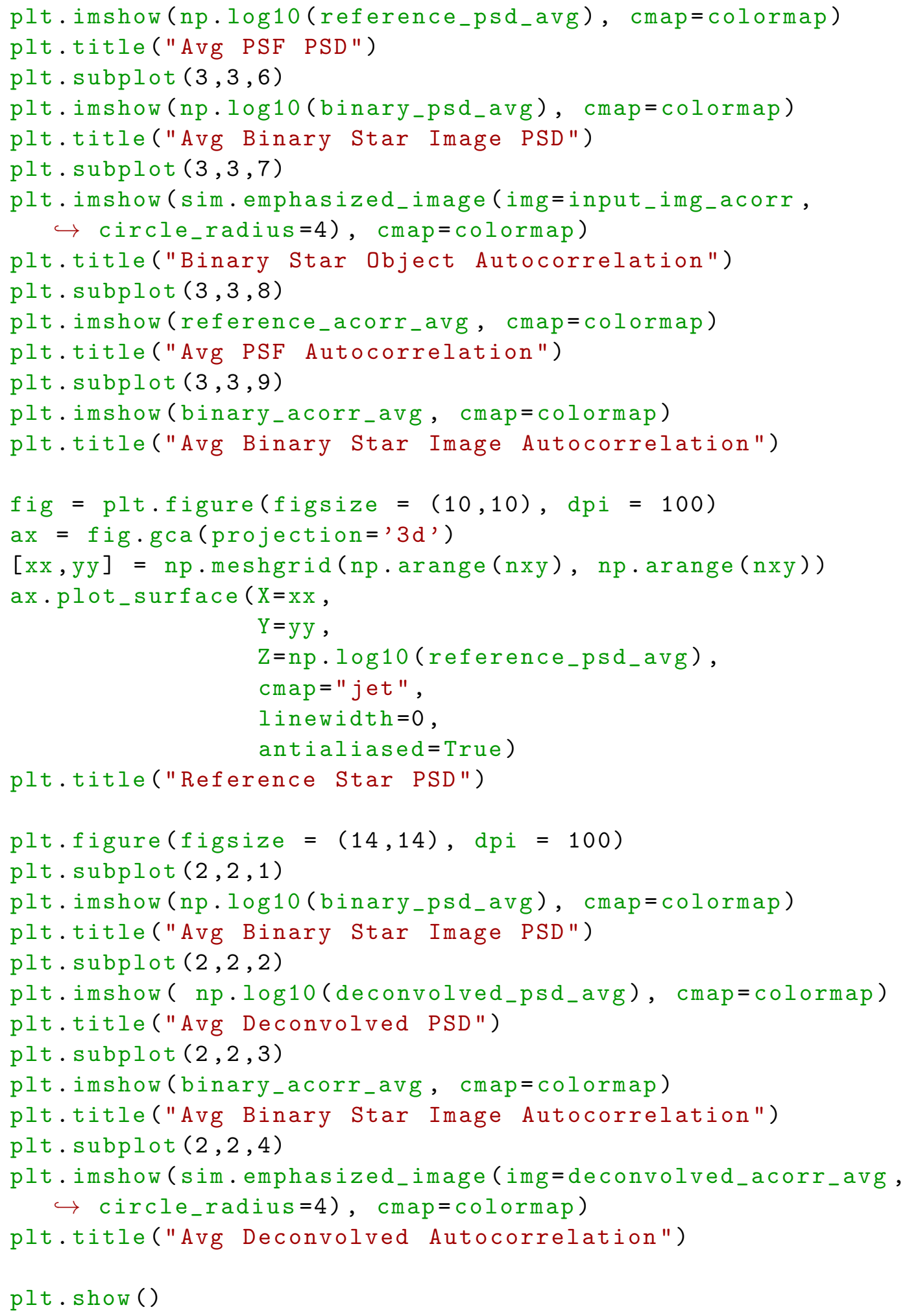

Simulation of Labeyrie deconvolution in the presence of noise:

\section{sim_wiener_deconv.py:}




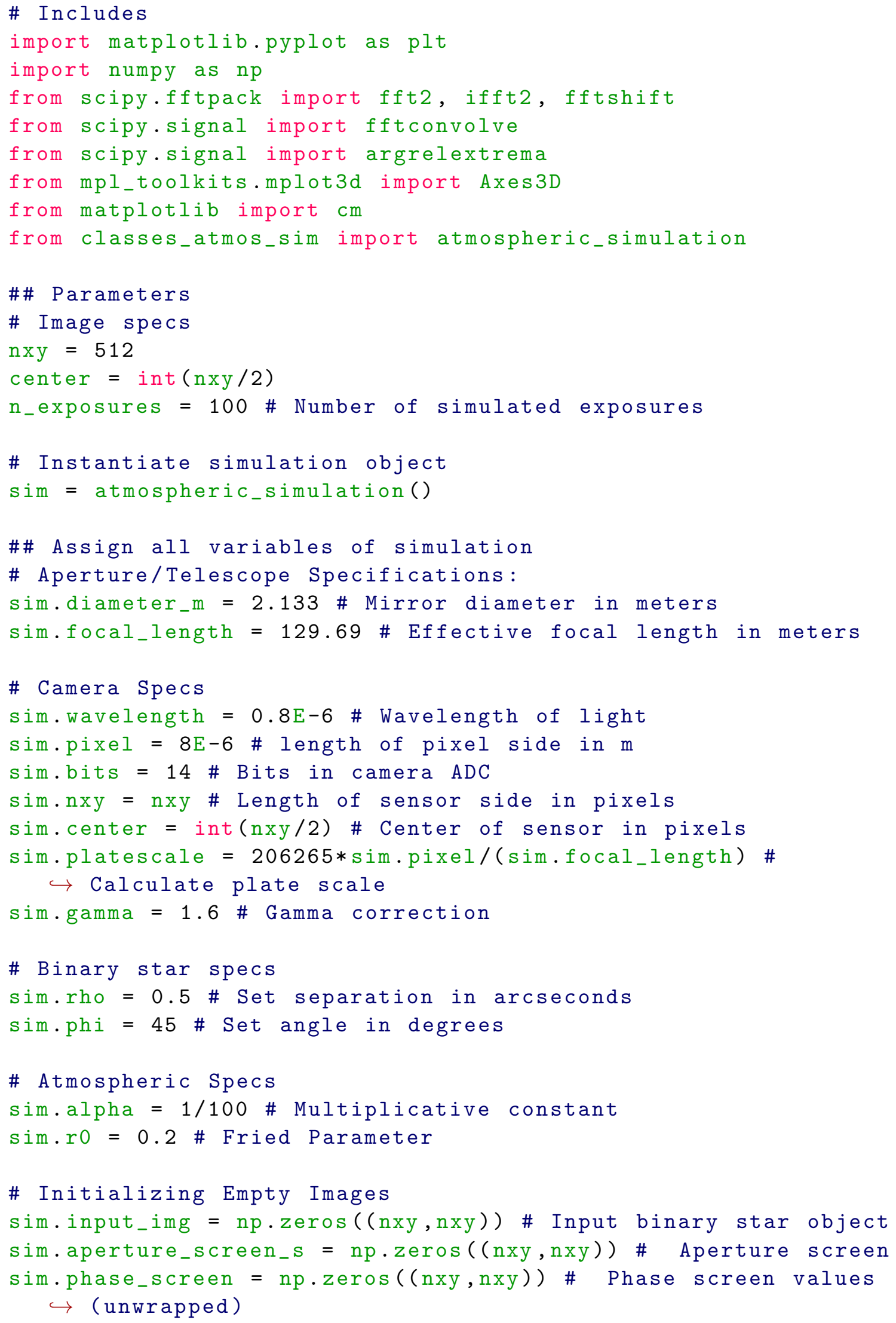




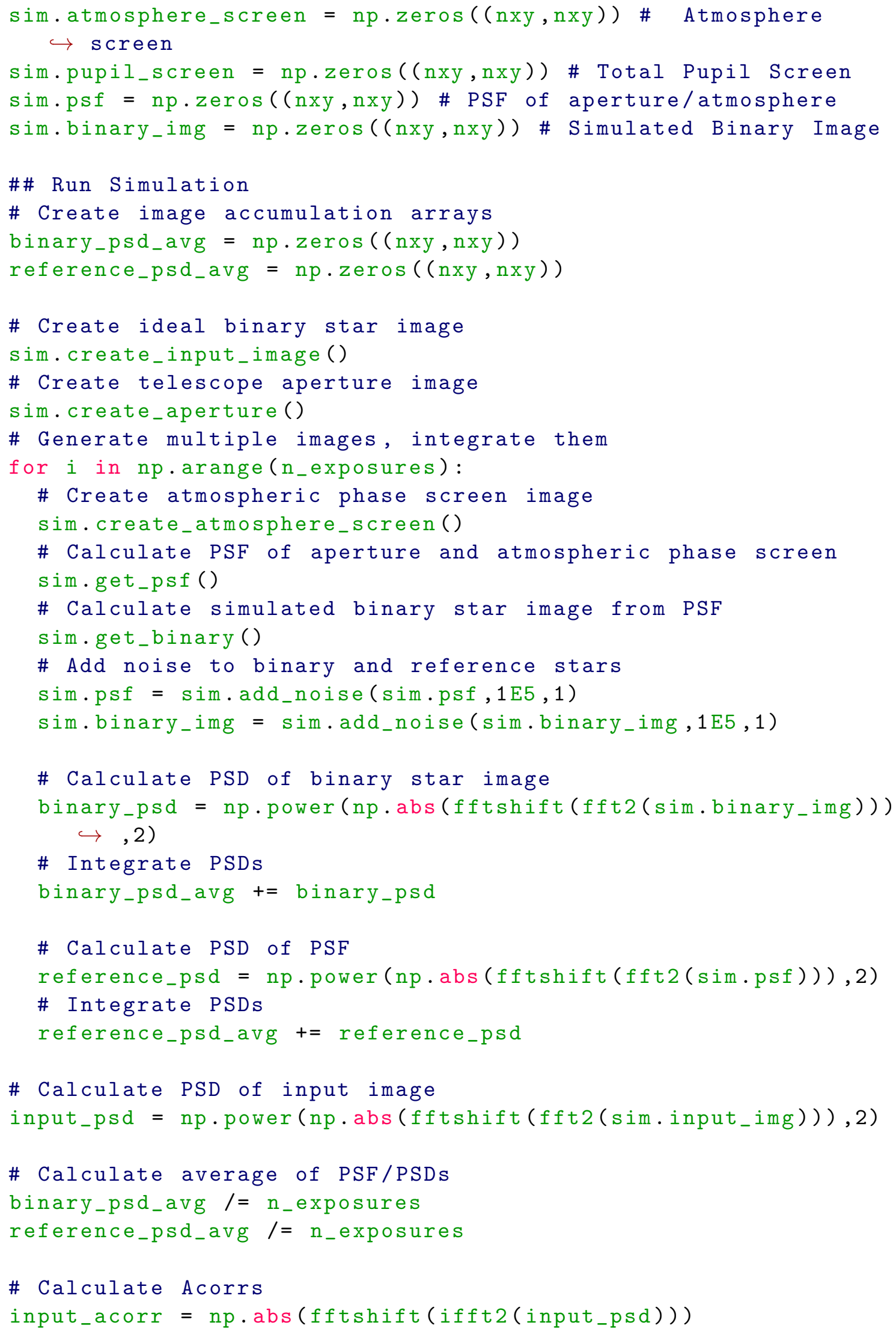




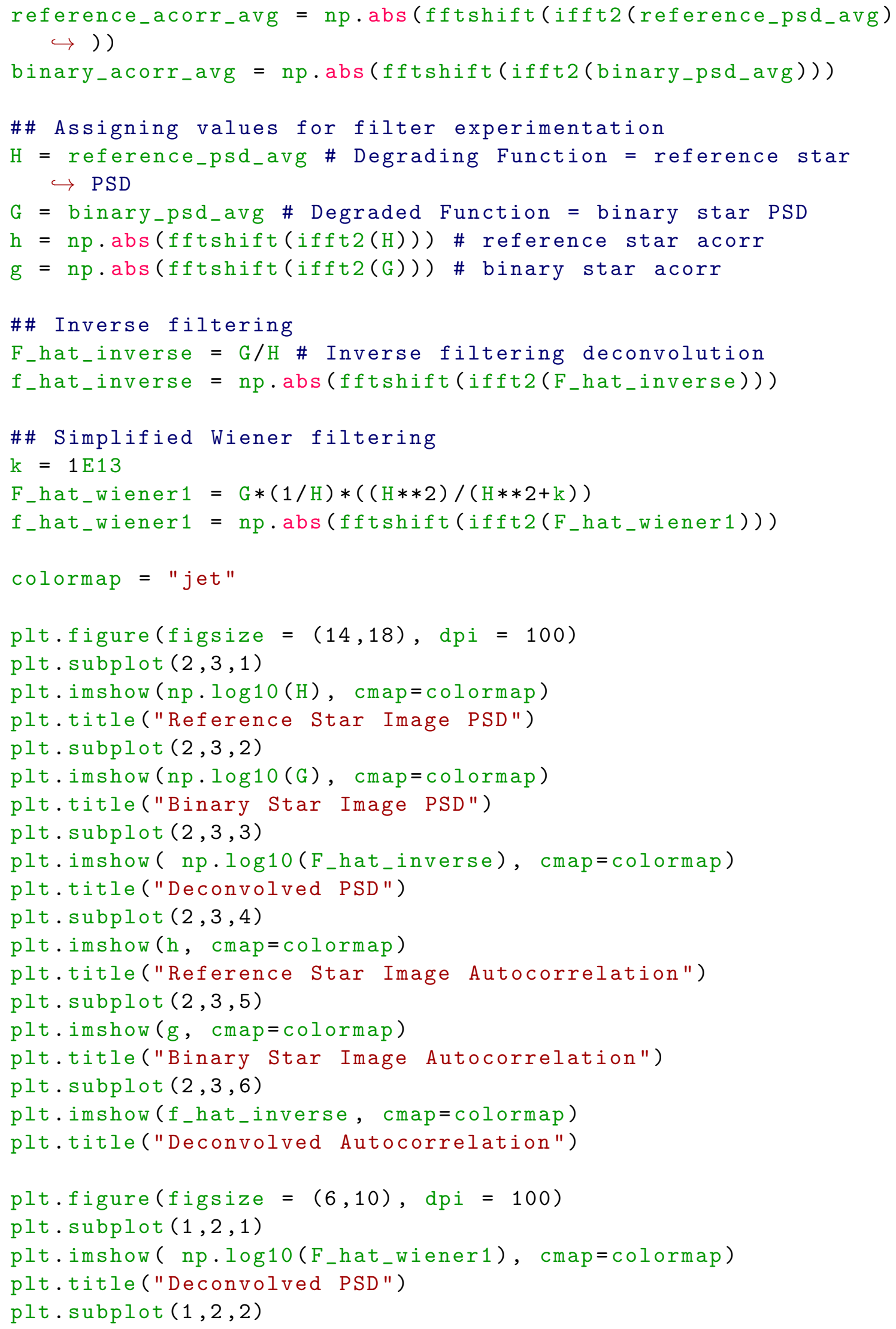




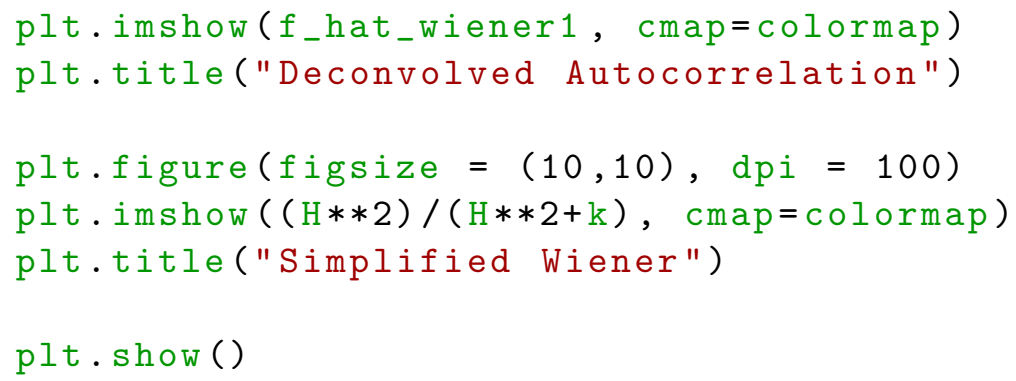

\section{Applying deconvolution to real data from KPNO. Uses Labeyrie Deconvolution}

\section{class shown in Appendix F:}

\section{sim_kpno_deconv.py:}

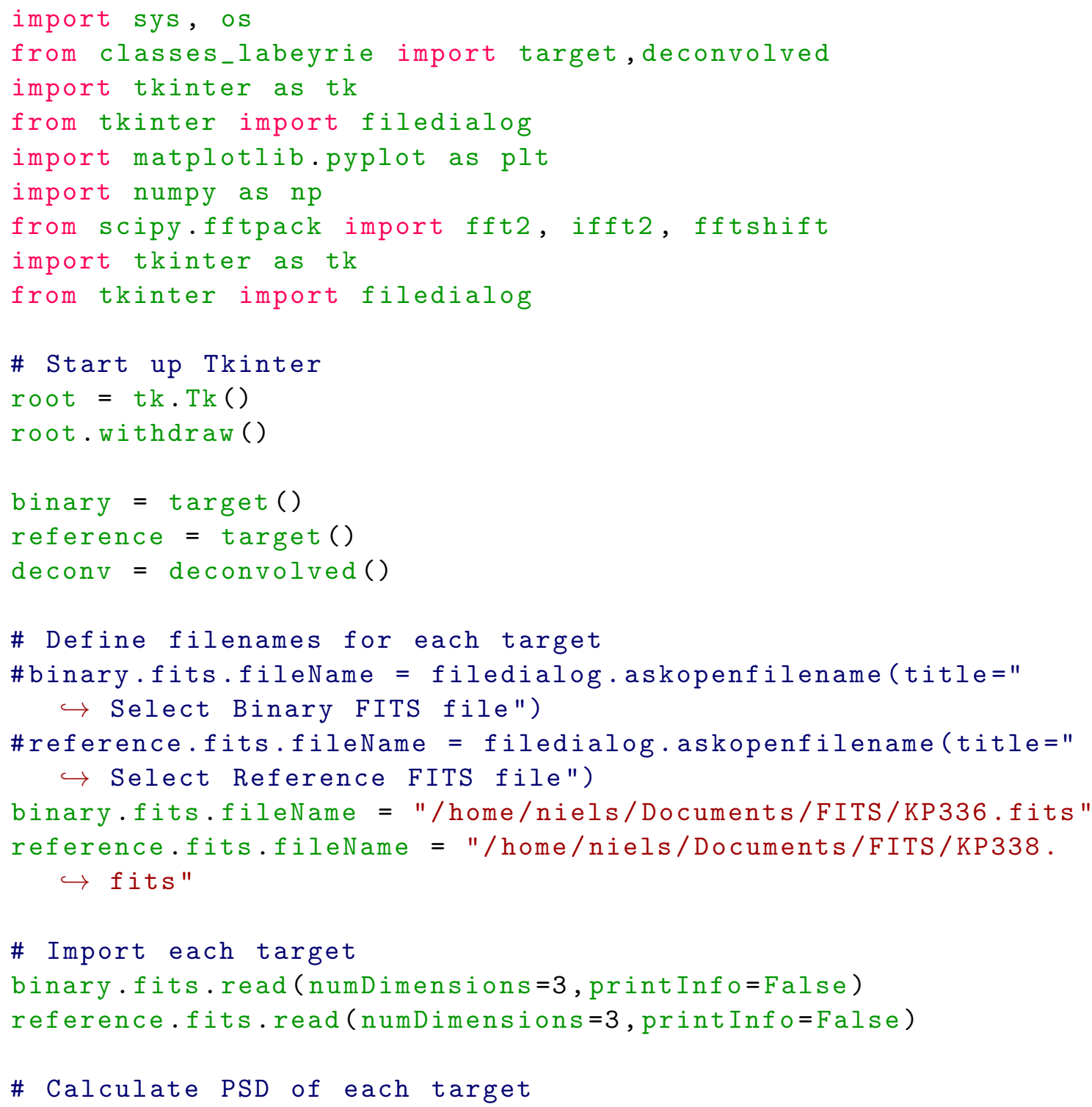




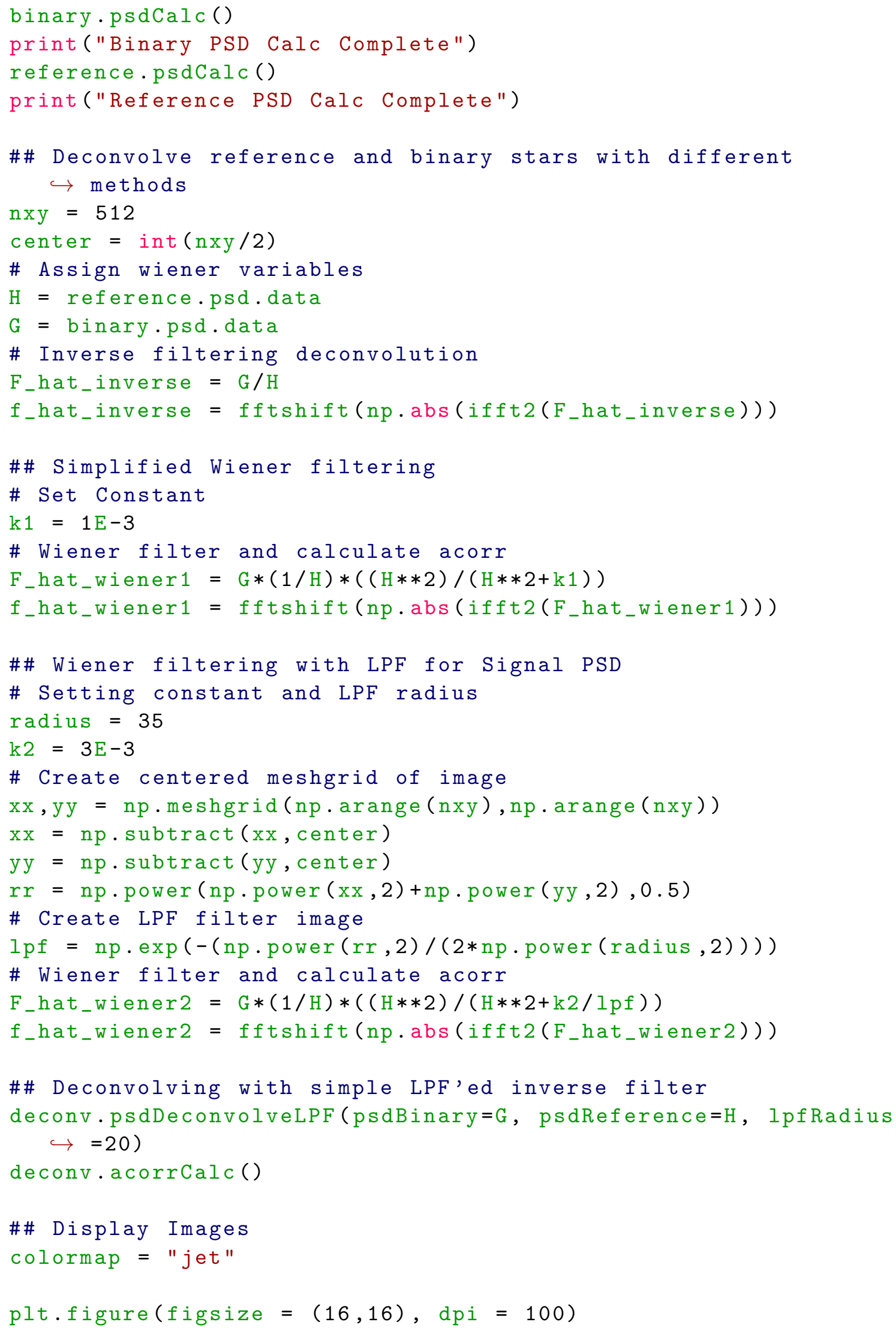




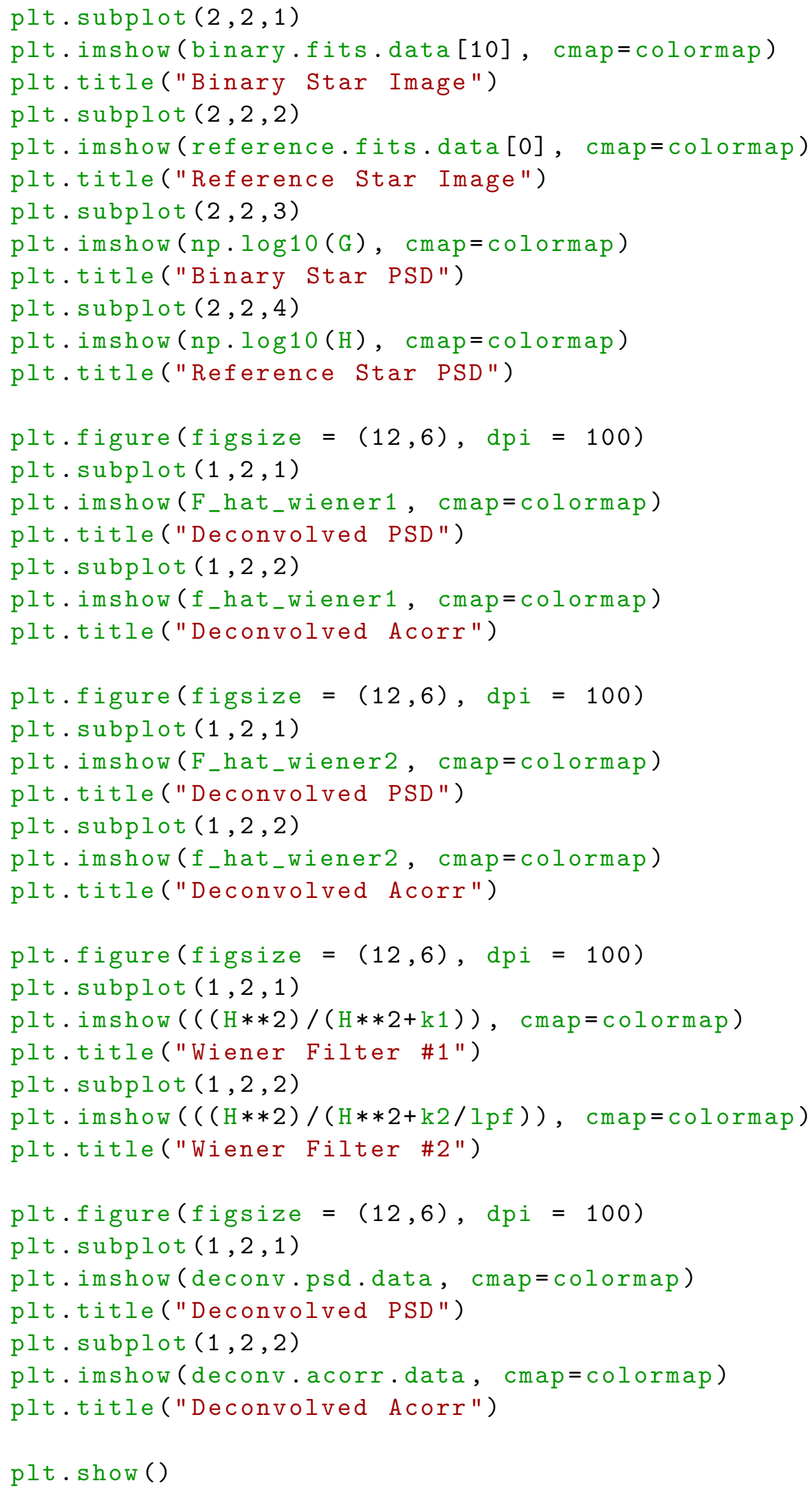




\section{F. Python Classes for Speckle Data Processing}

\section{classes_labeyrie.py:}

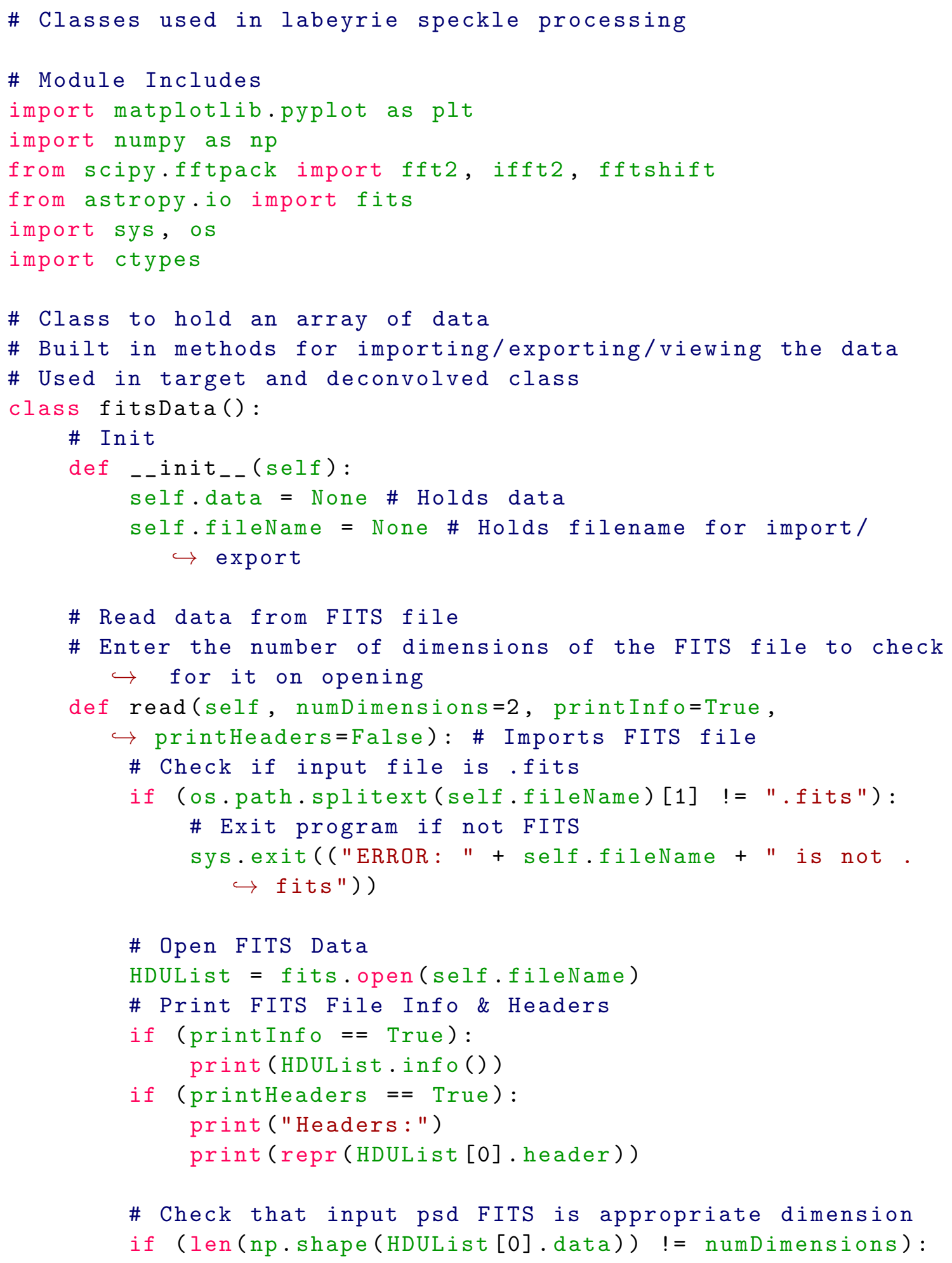




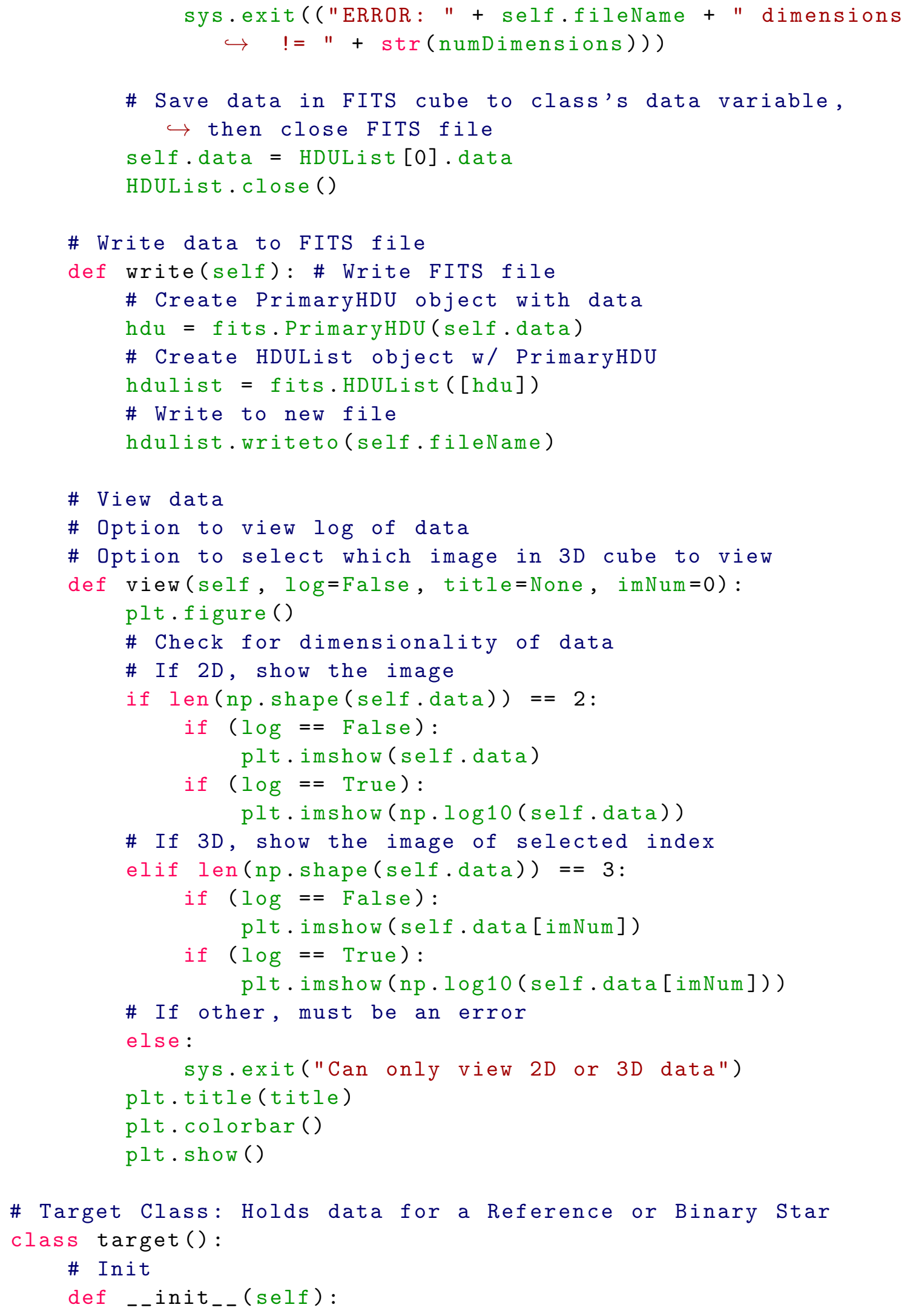




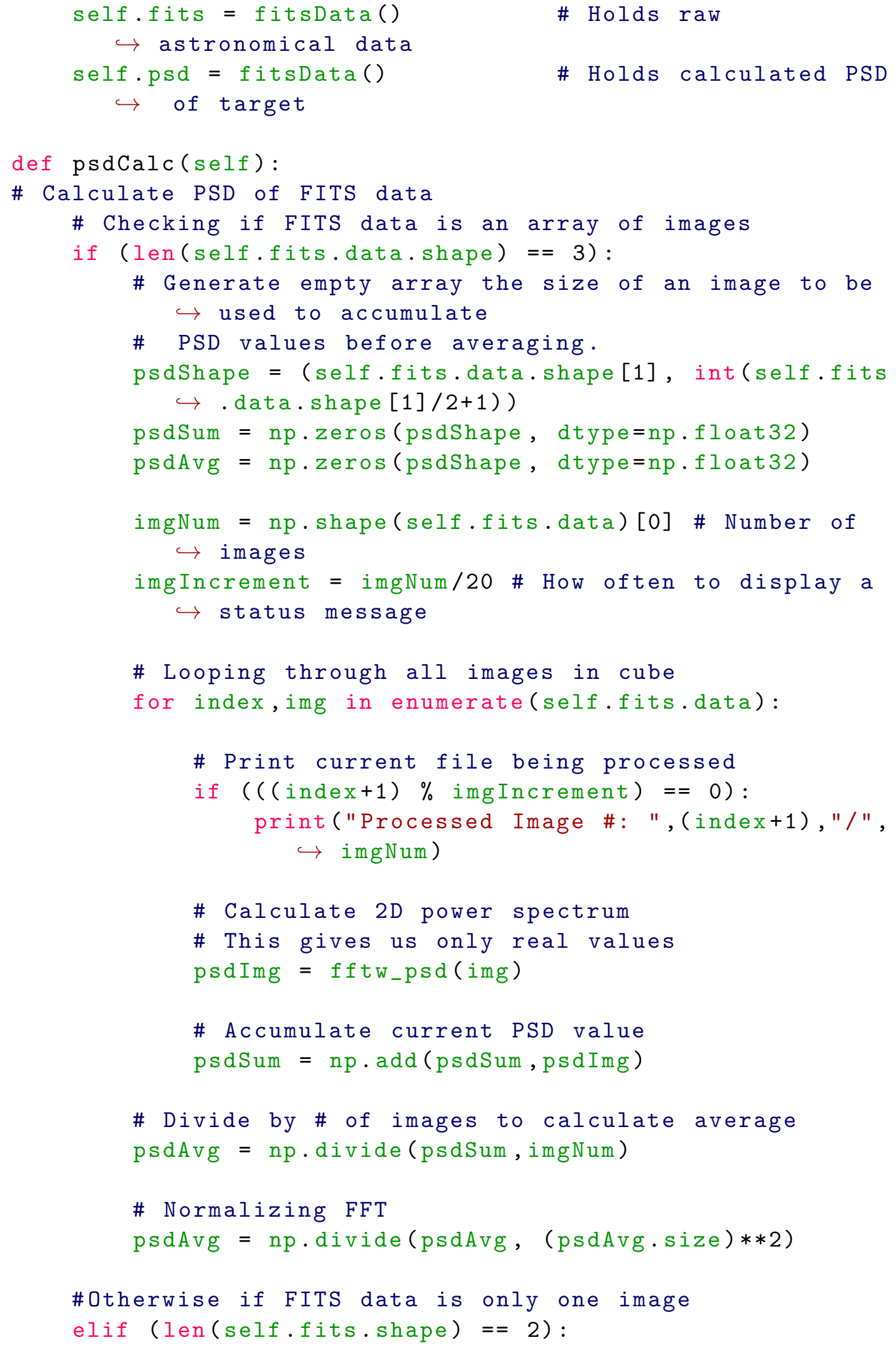




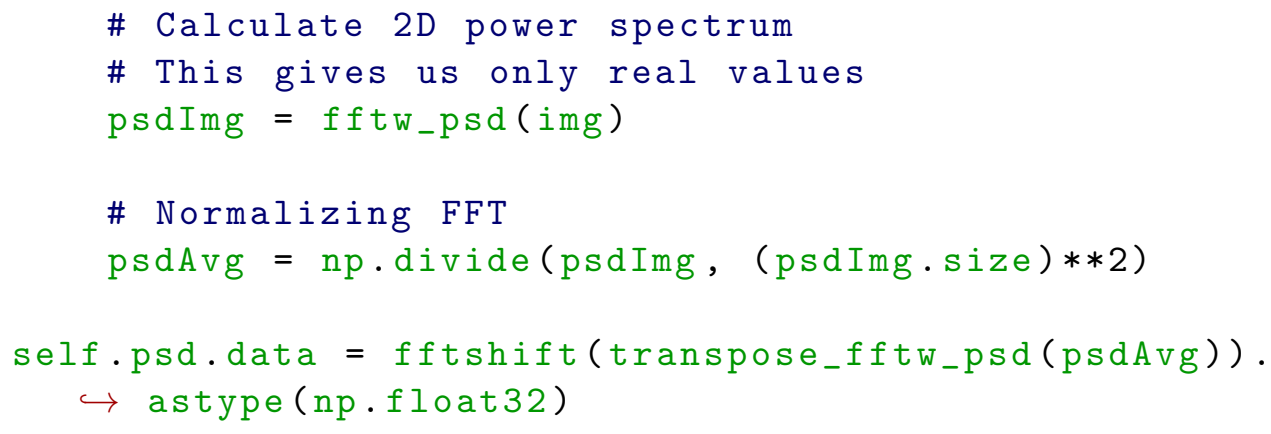

\# Deconvolve PSDs 


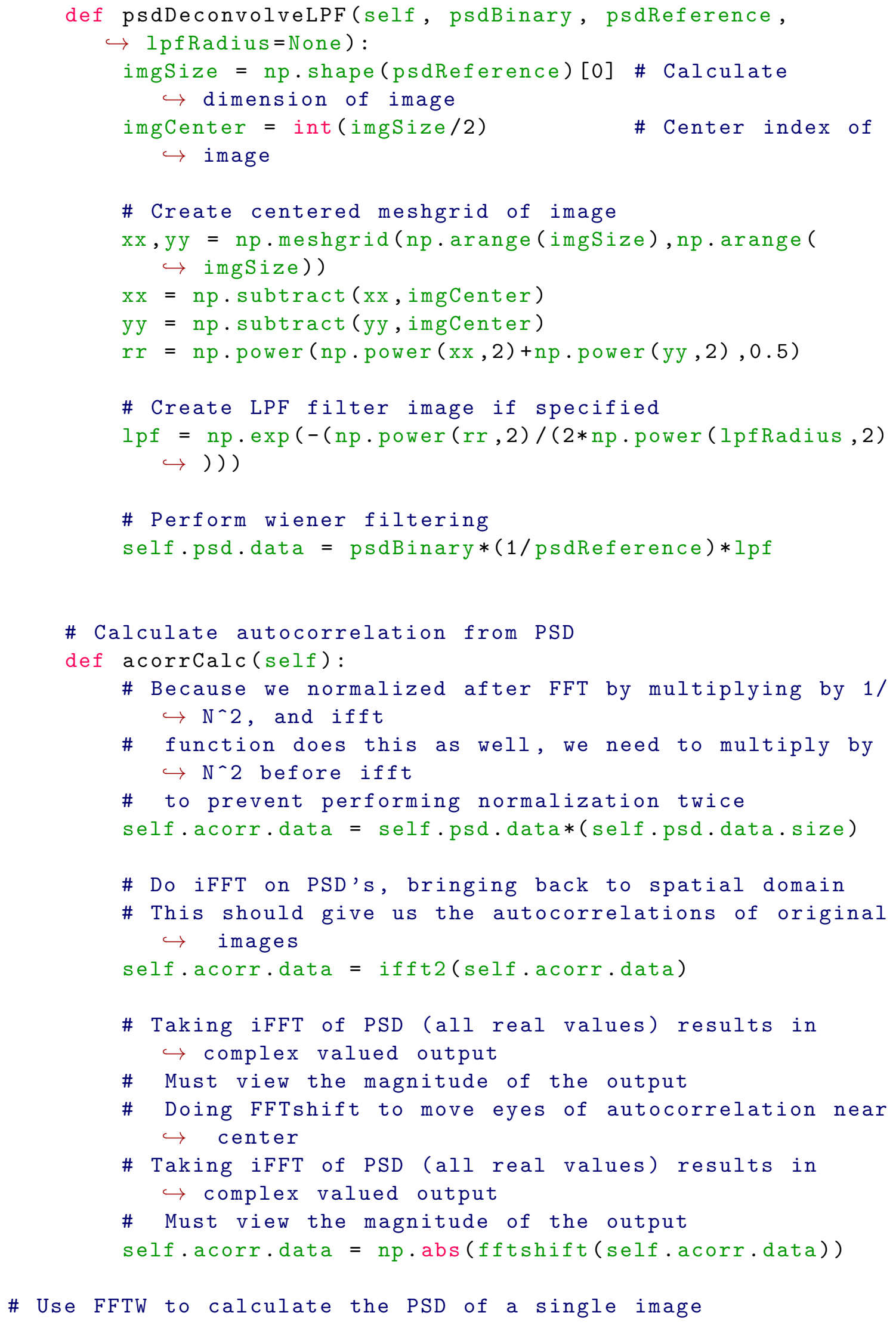




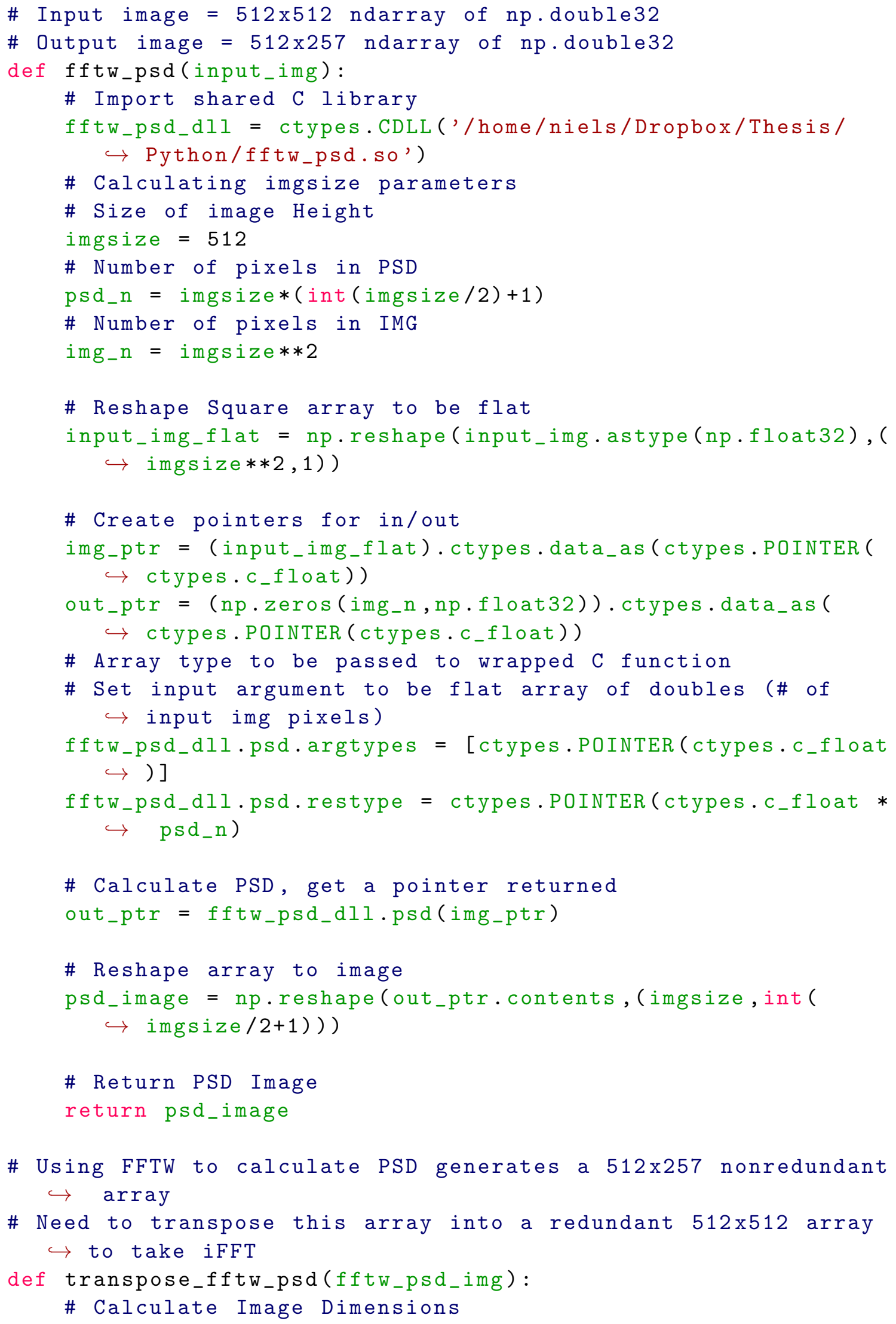




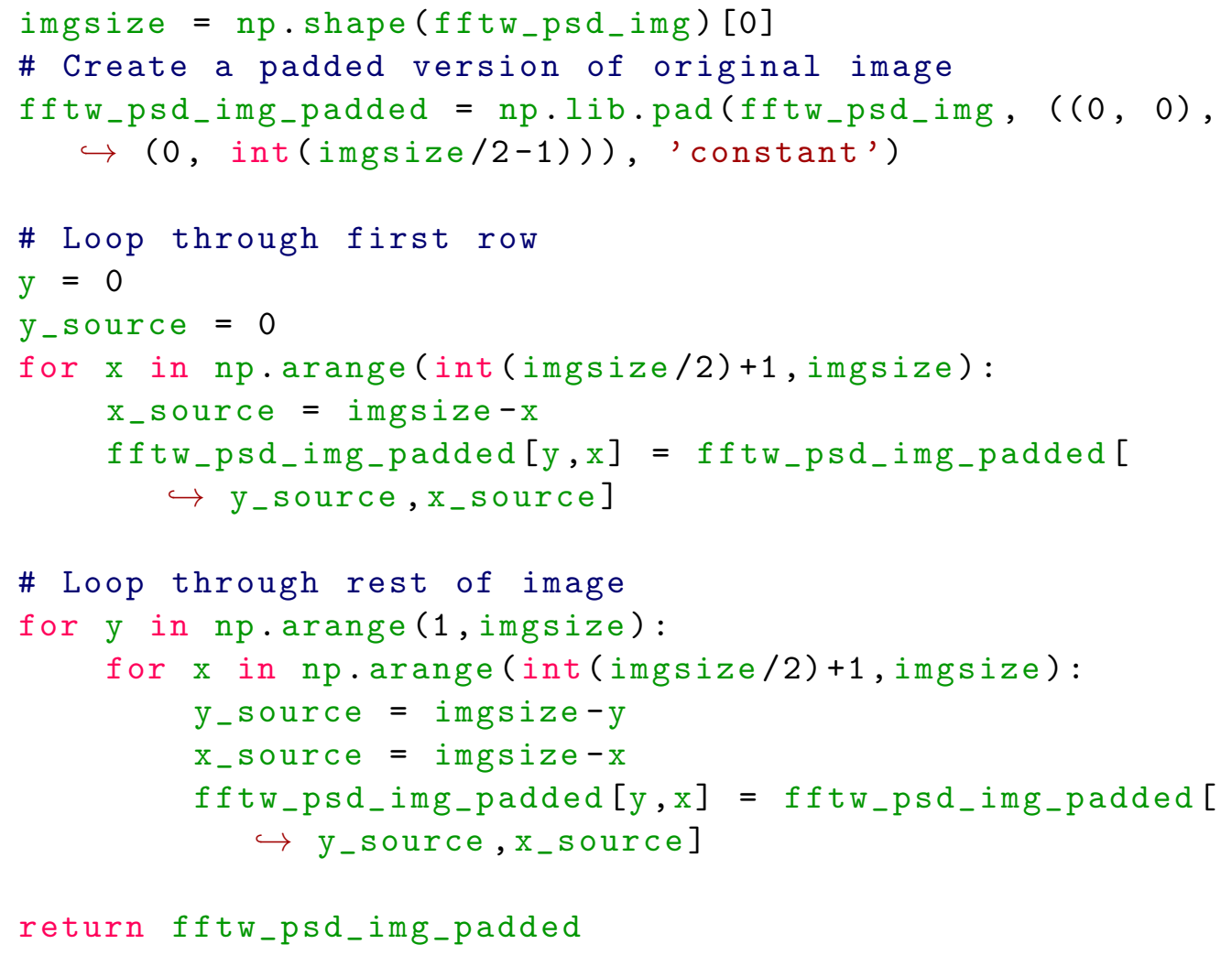

\section{classes_astrometry.py:}

\# Module Includes

import matplotlib.pyplot as plt

import numpy as np

from scipy.fftpack import fft 2 , ifft 2 , fftshift

from astropy.io import fits

import sys, os, cv2

import ctypes

\# coords class: Holds methods for working in polar/cart $\hookrightarrow$ cordinates

\# Used in camsky class

class coords ():

def _-_init_- (self, midpoint):

self.theta $=$ None

self.rho = None

self.x $x$ None

self.y $=$ None

self.midpoint $=$ midpoint

\# Orbital plot has odeg pointing down on the screen [ $\hookrightarrow$ resulting in 90 deg shift]

\# Theta rotates counterclockwise from odeg 


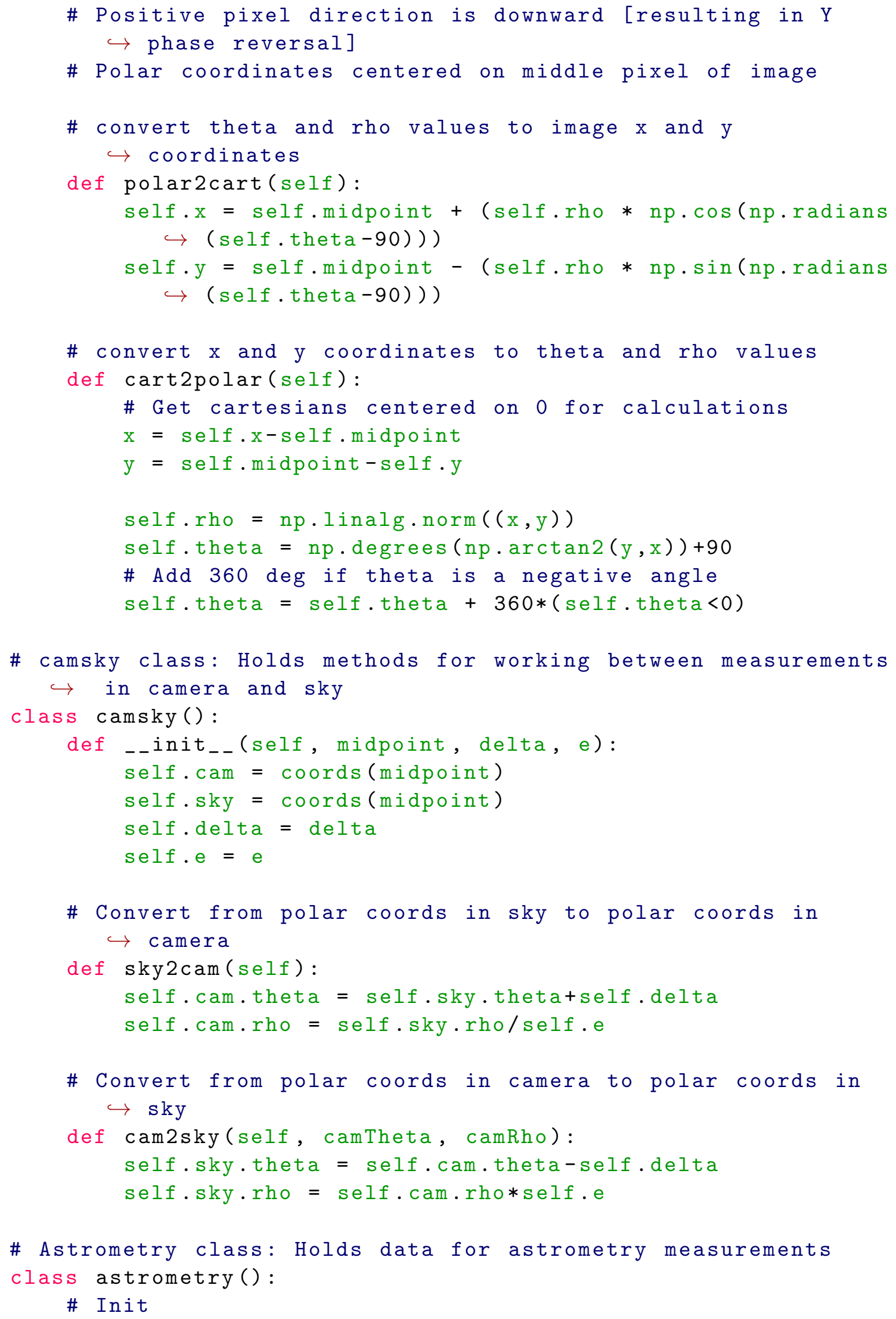




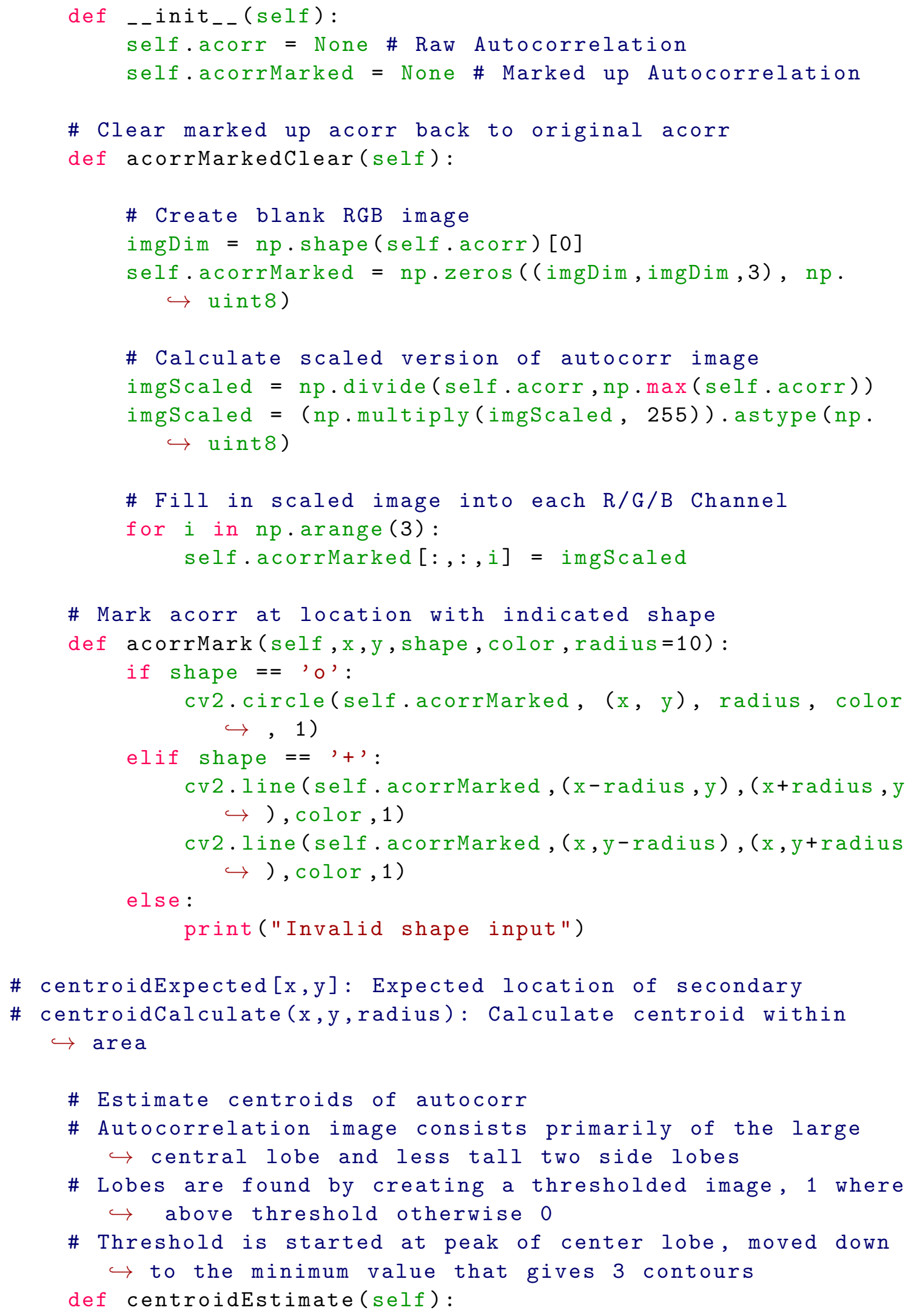




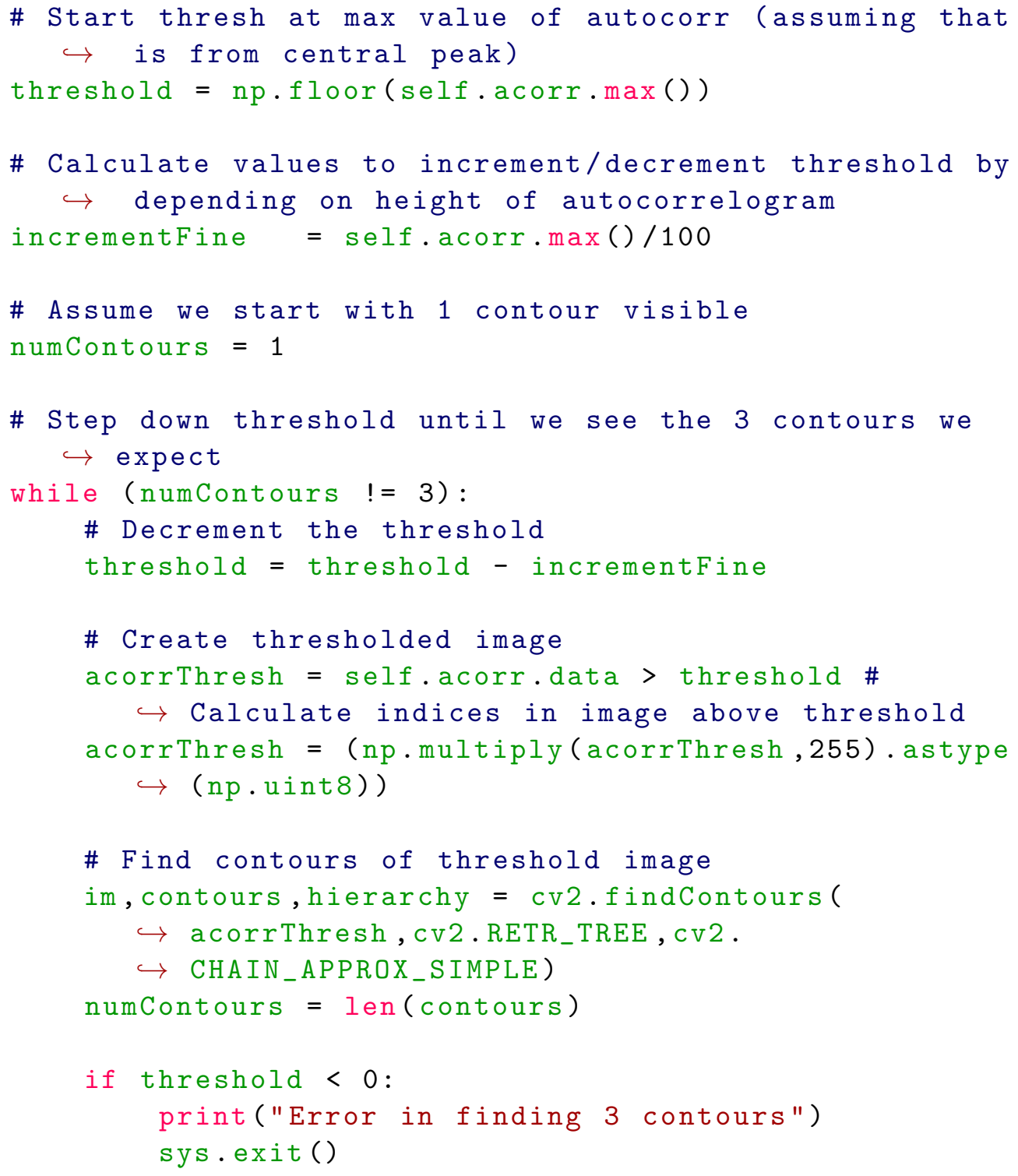




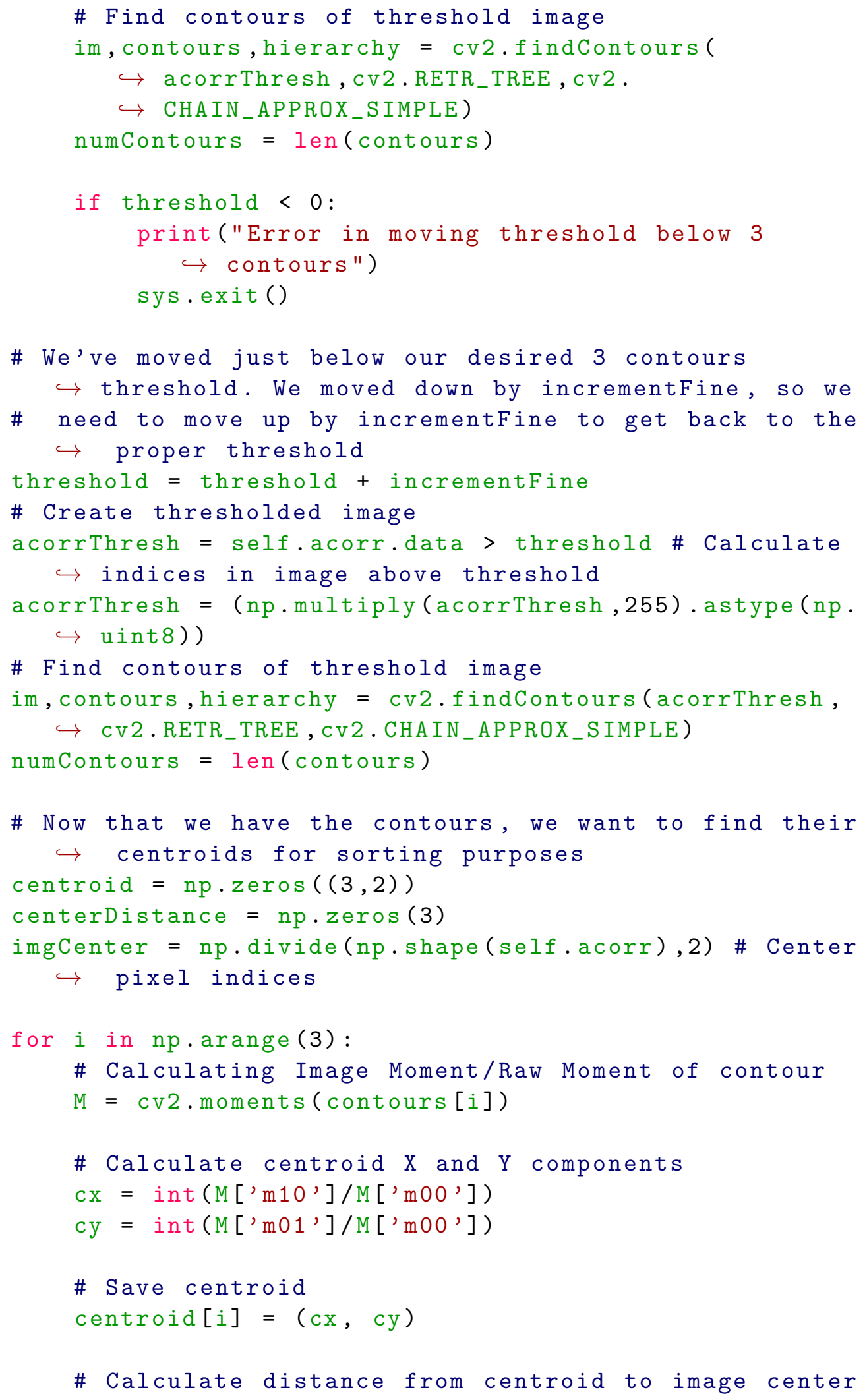




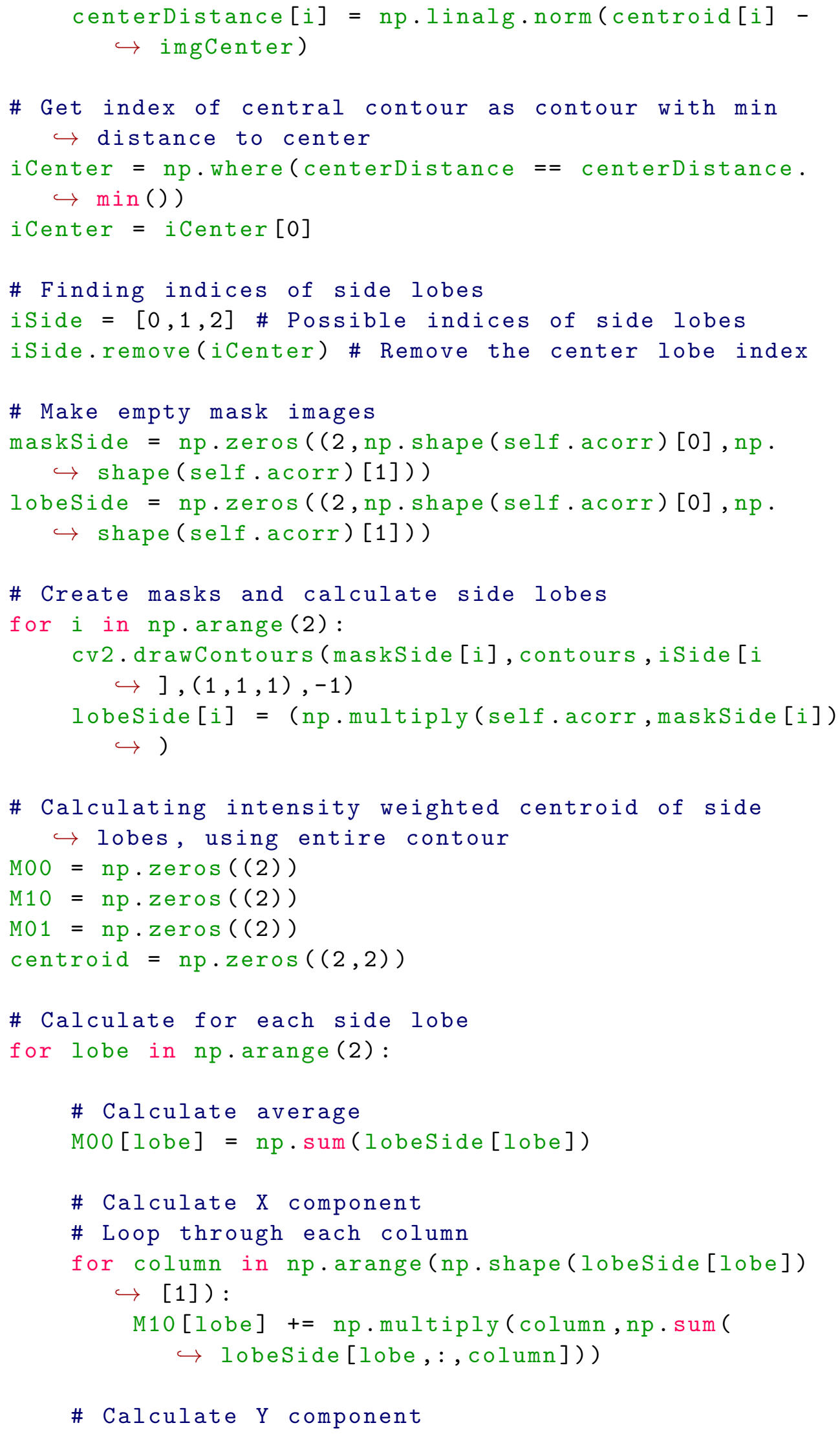




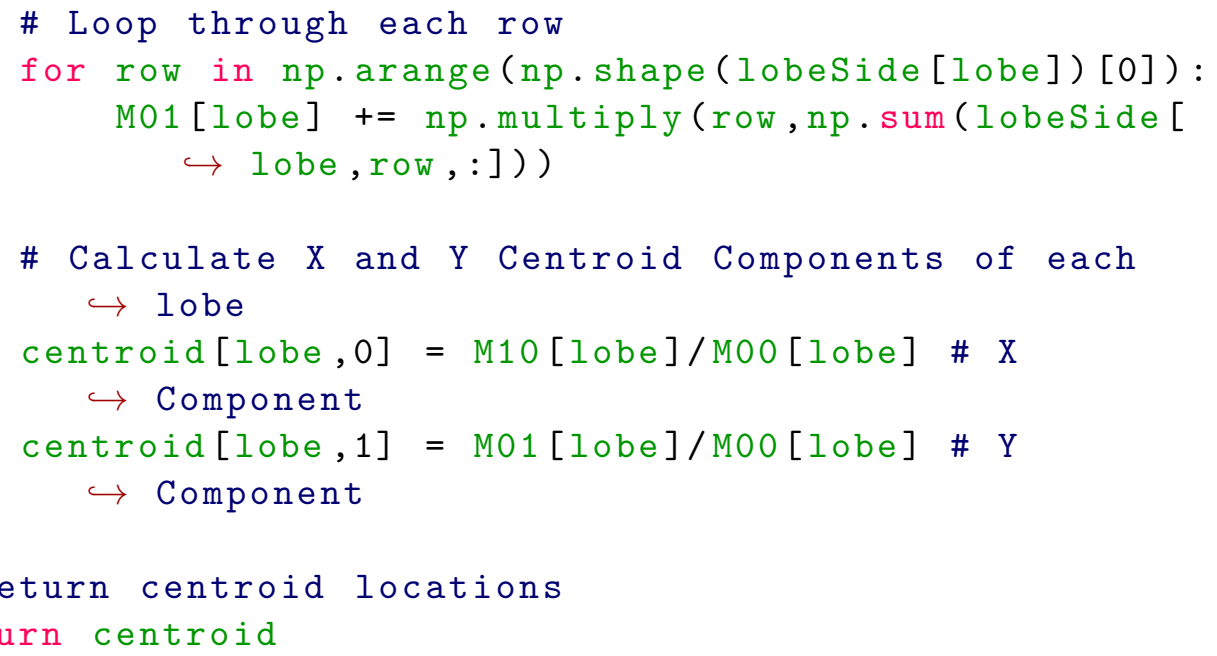




\section{G. Processing FITS Data using classes_labeyrie.py}

\section{Preprocessing done in labeyrie_preprocess.py:}

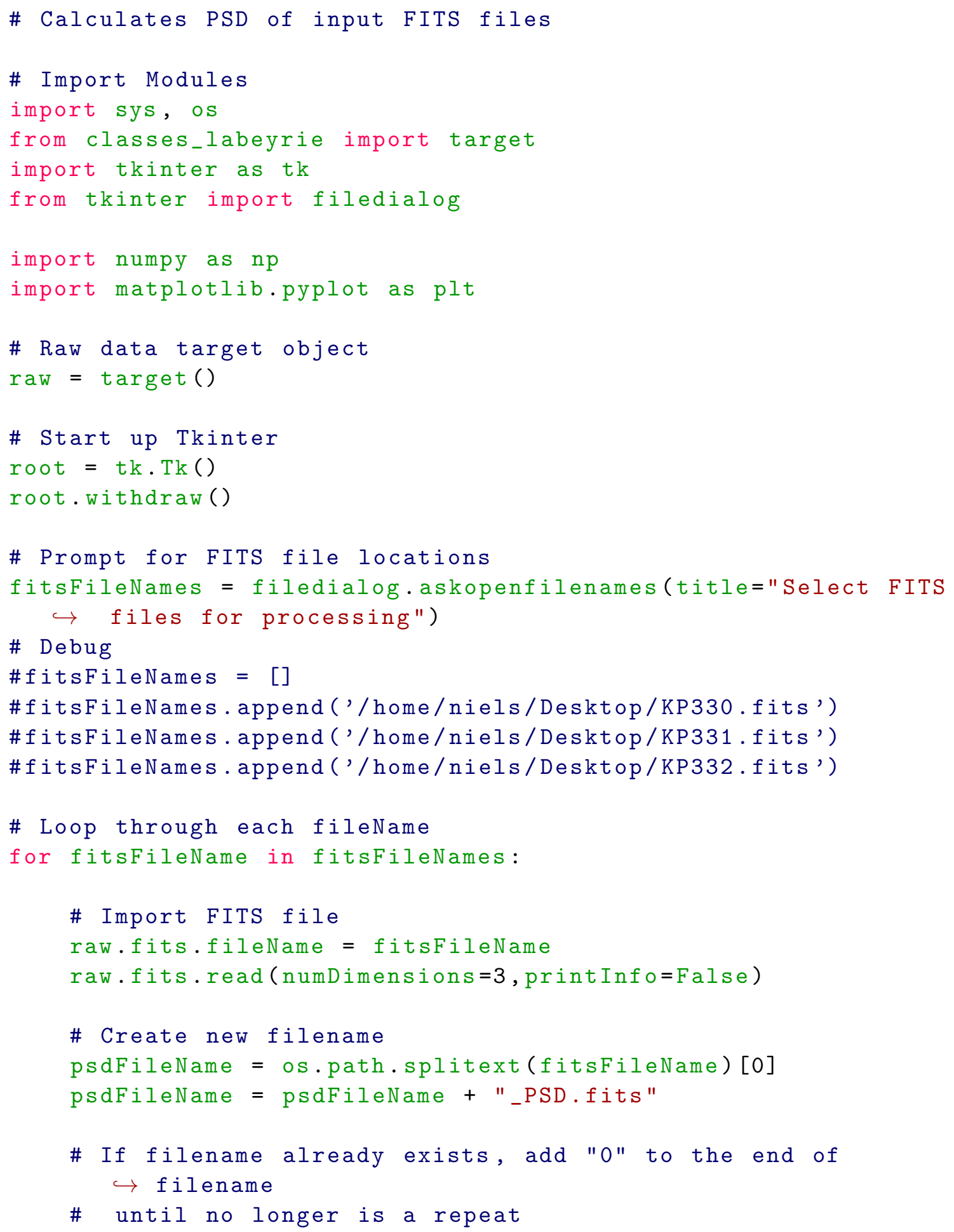




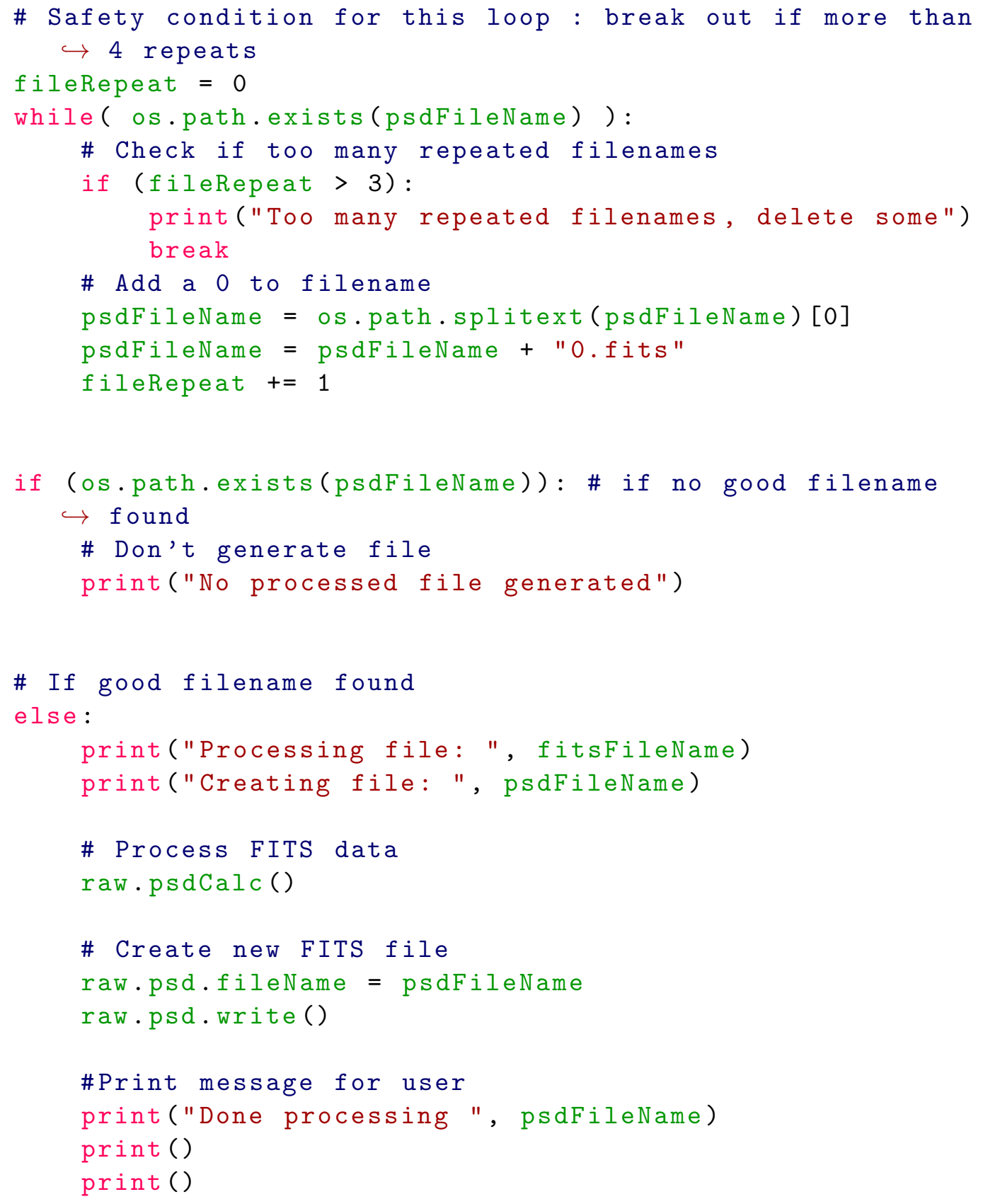

\section{Deconvolution done in labeyrie_deconv.py:}

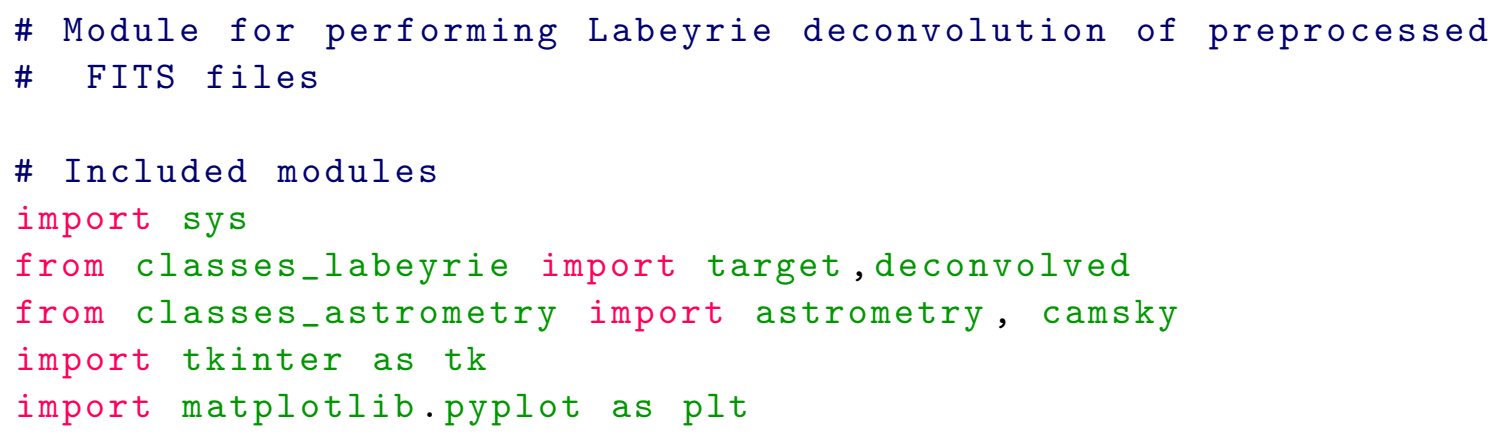




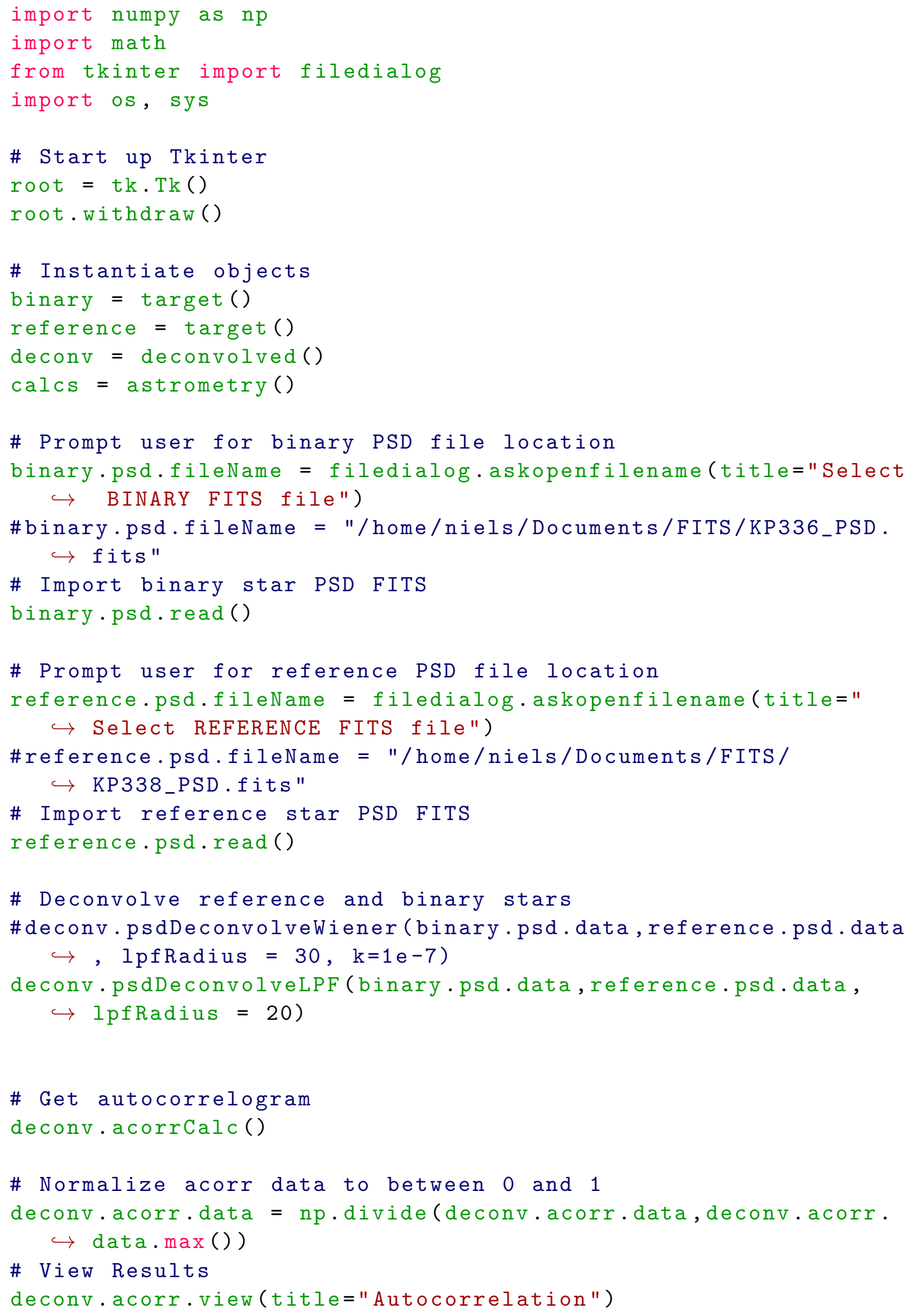




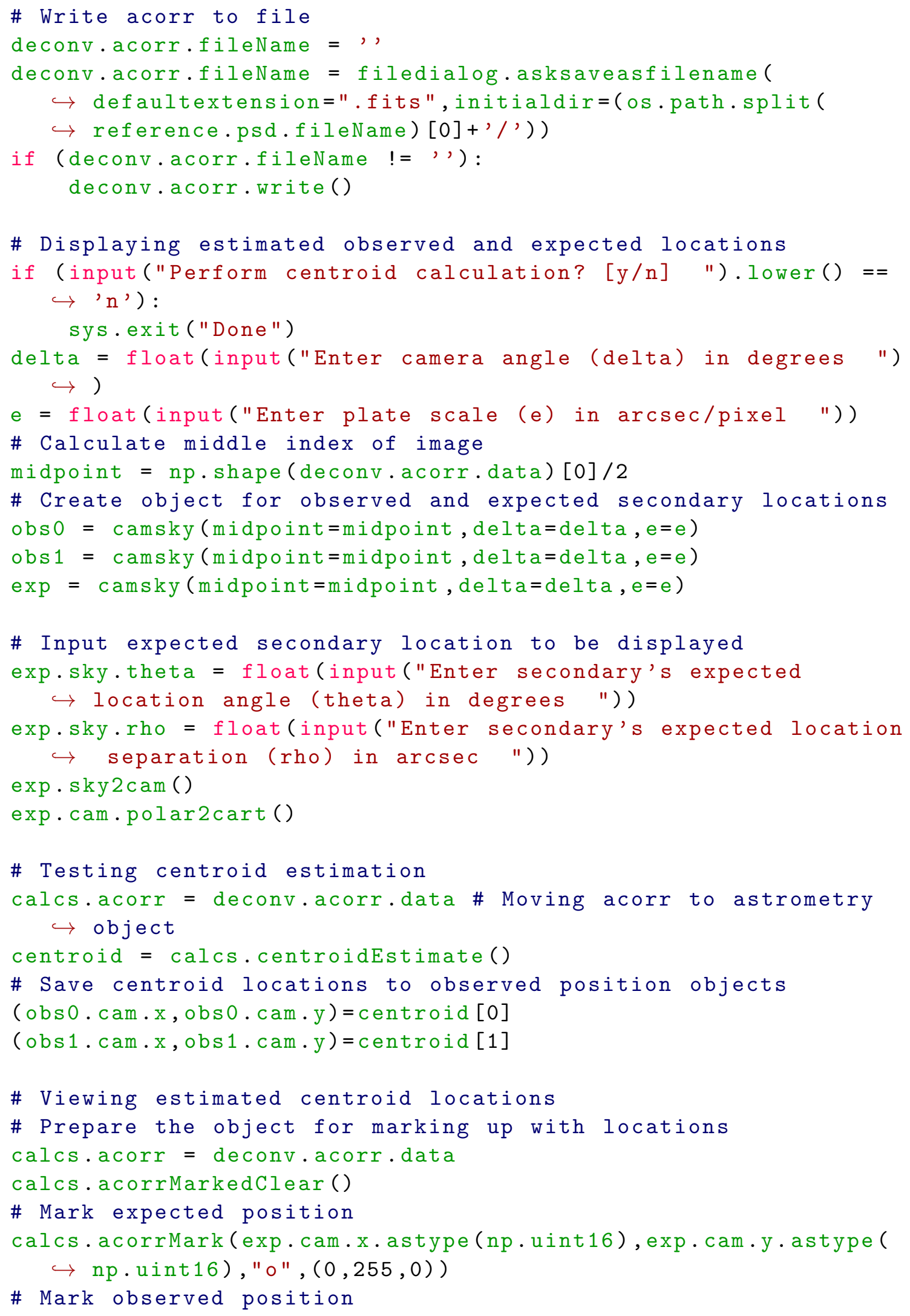


calcs.acorrMark (obs0.cam.x.astype (np.uint16), obs0.cam.y.astype $\hookrightarrow(\mathrm{np} \cdot$ uint 16$), " \circ ",(255,0,0))$

calcs.acorrMark (obs1.cam.x.astype (np.uint16), obs1.cam.y.astype $\hookrightarrow(\mathrm{np} \cdot$ uint 16$), " \circ ",(255,0,0))$

\# View Image

plt.figure()

plt.imshow(calcs.acorrMarked)

plt.title("Observed and expected secondary locations")

plt.show () 


\section{H. Benchmarking PSD Computation Methods}

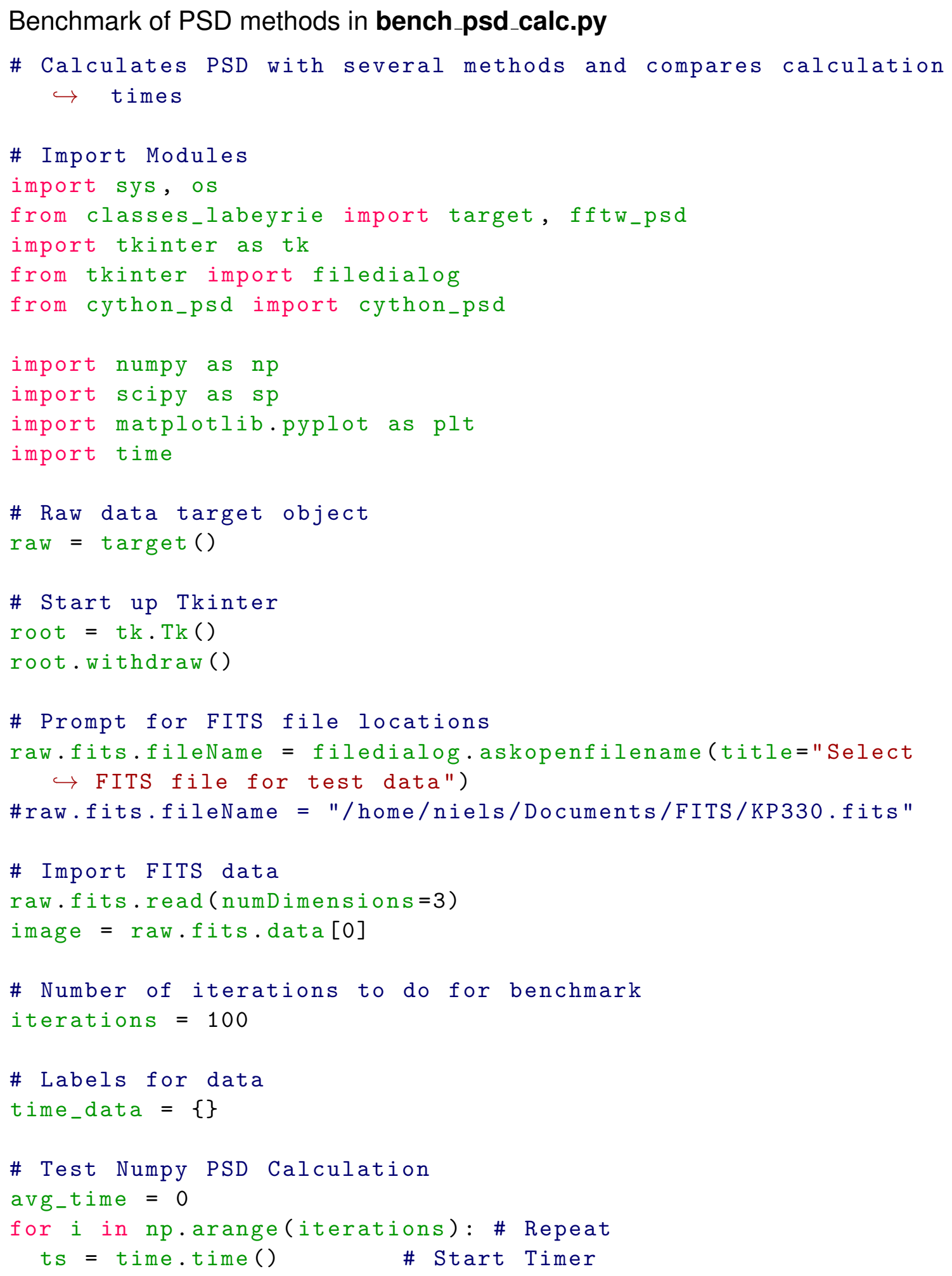




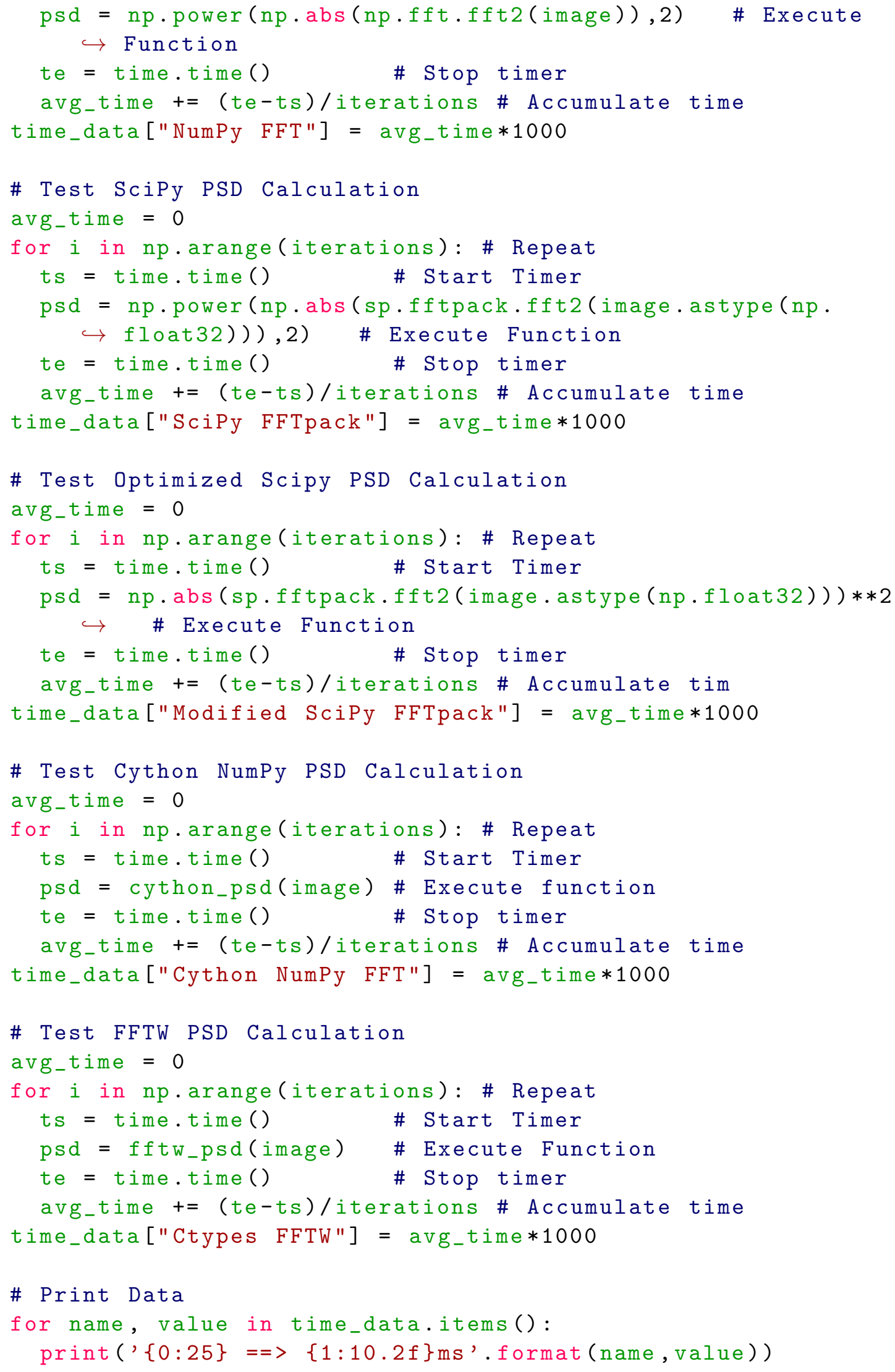




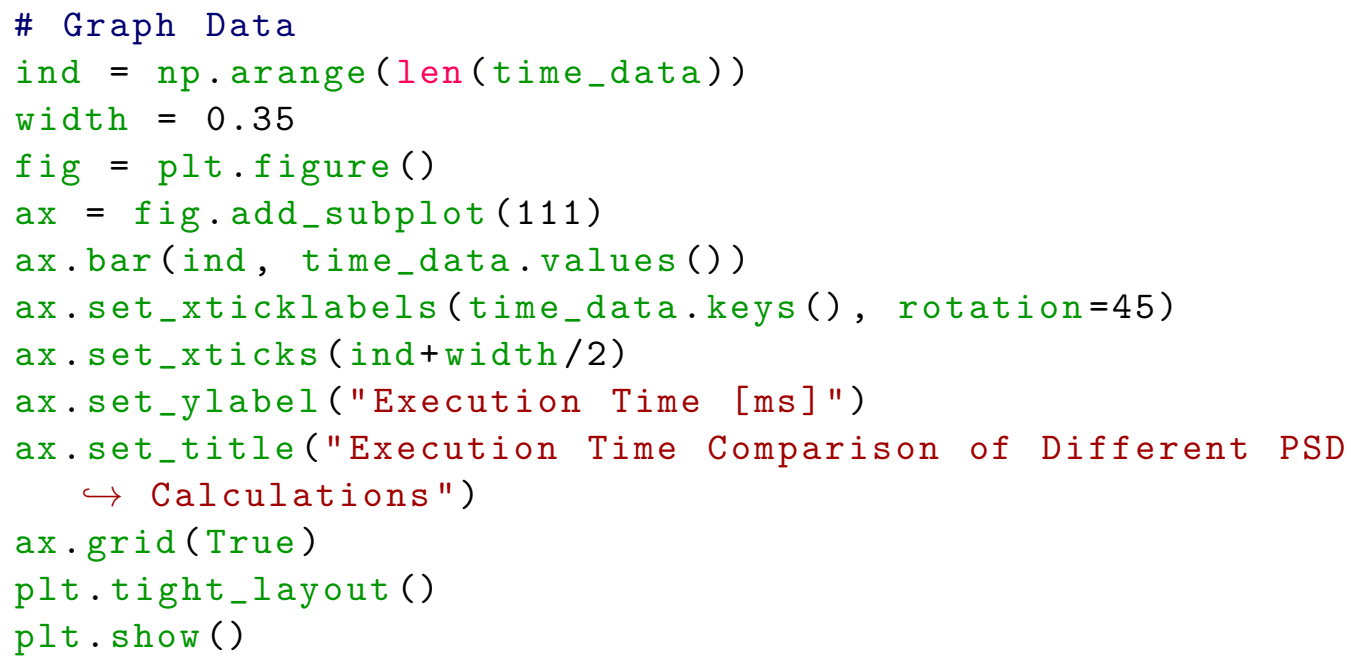

\section{Cython PSD Function generated from cython psd.pyx file}

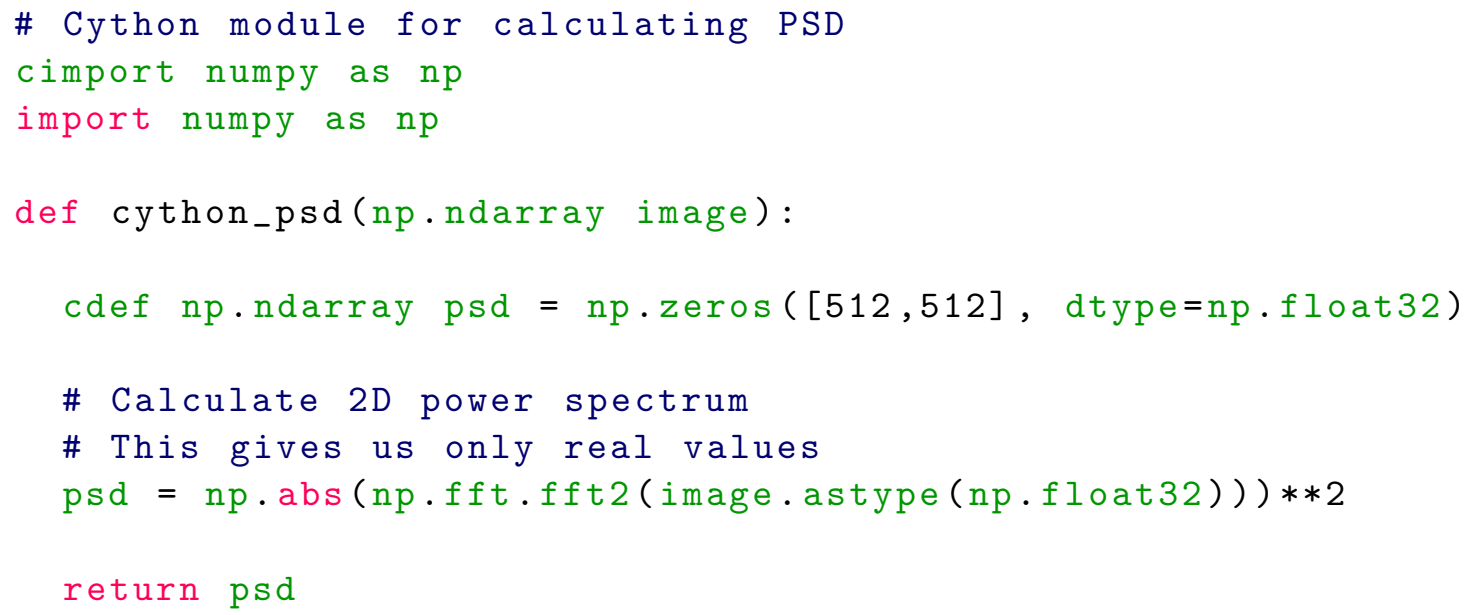

\section{Optimized FFTW C Code in fftw_psd.c}

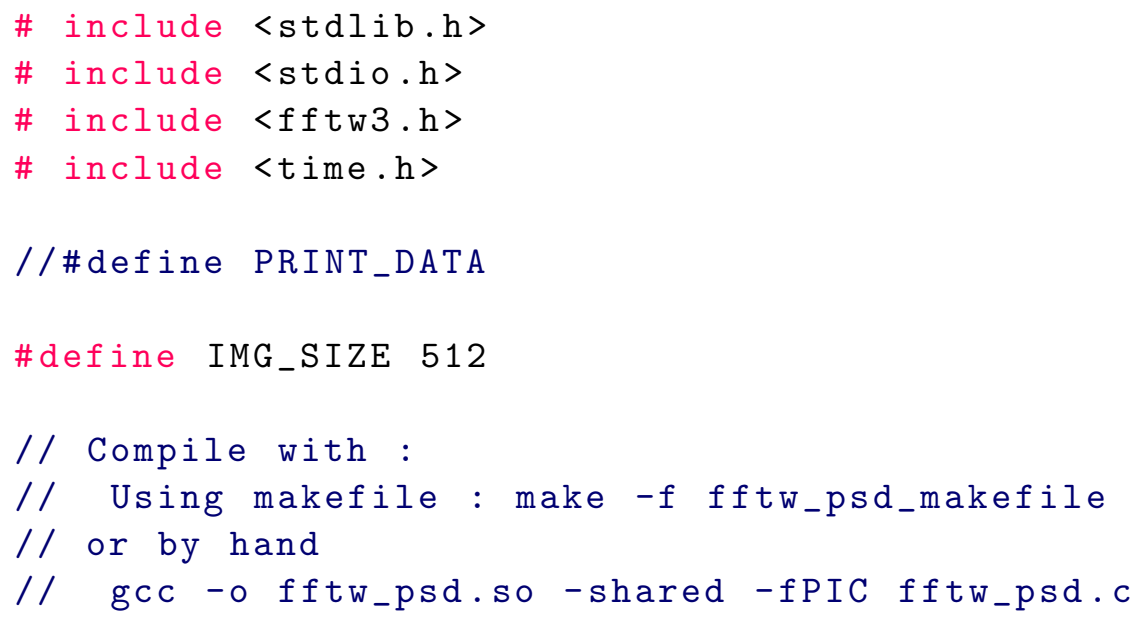




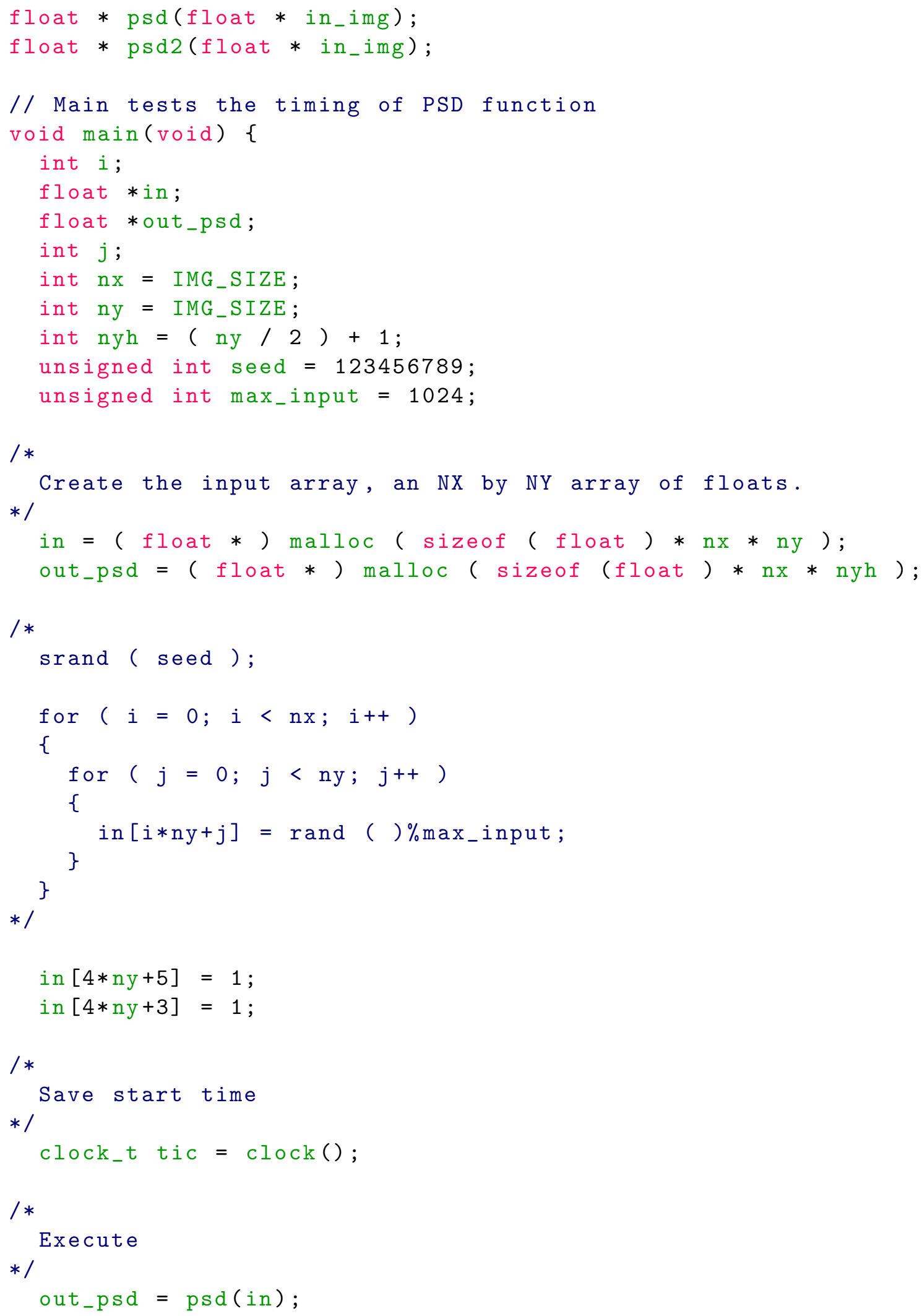




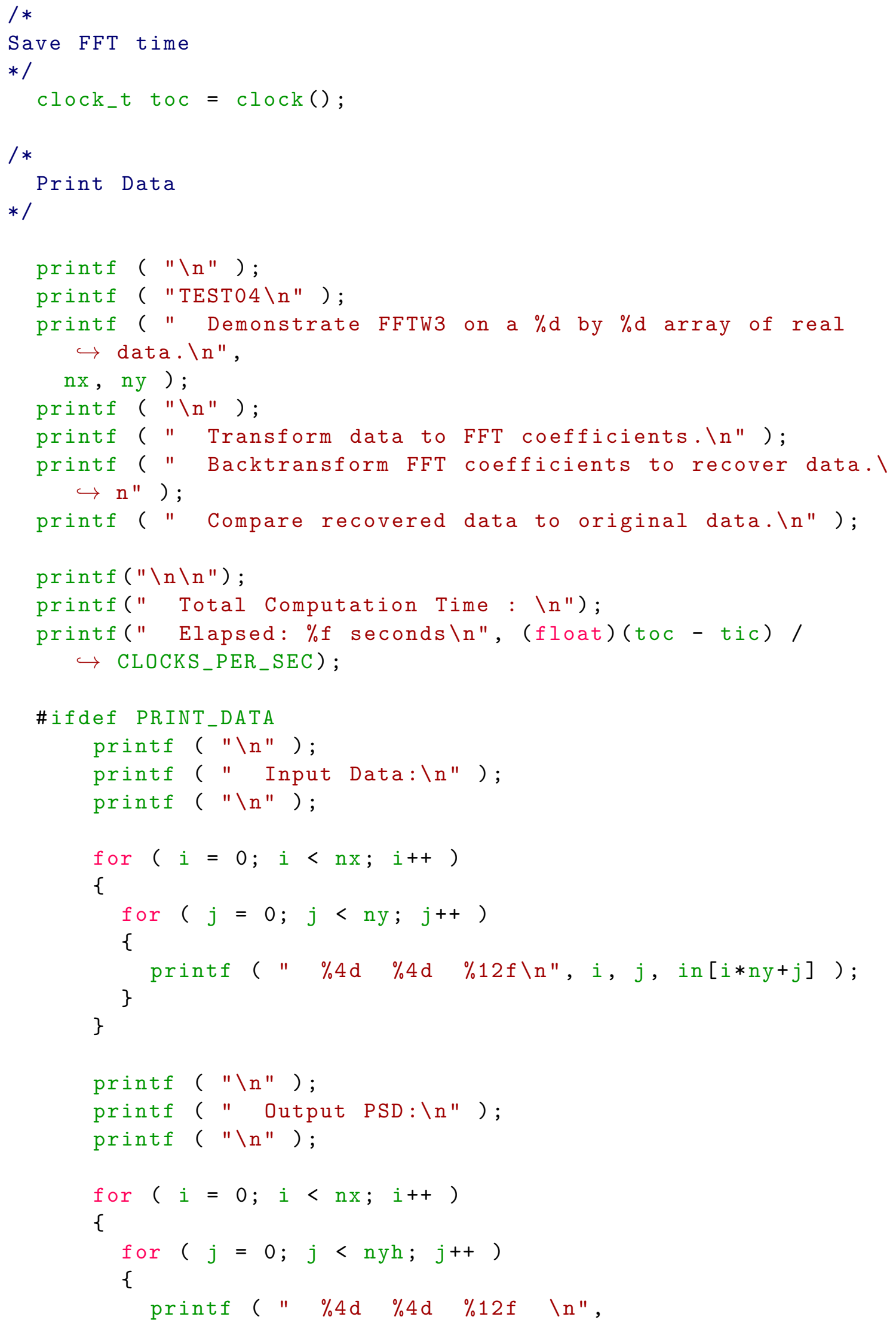




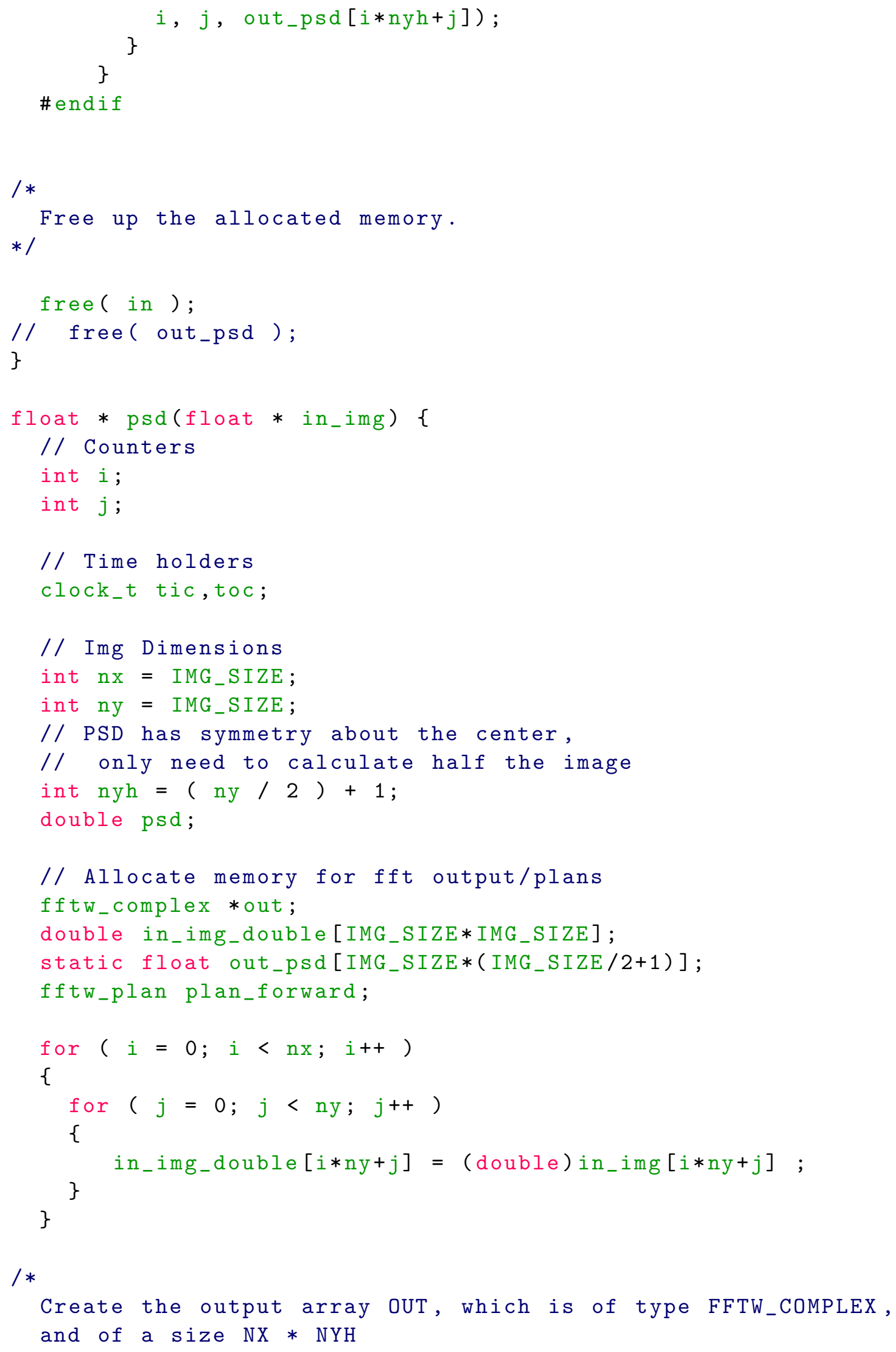




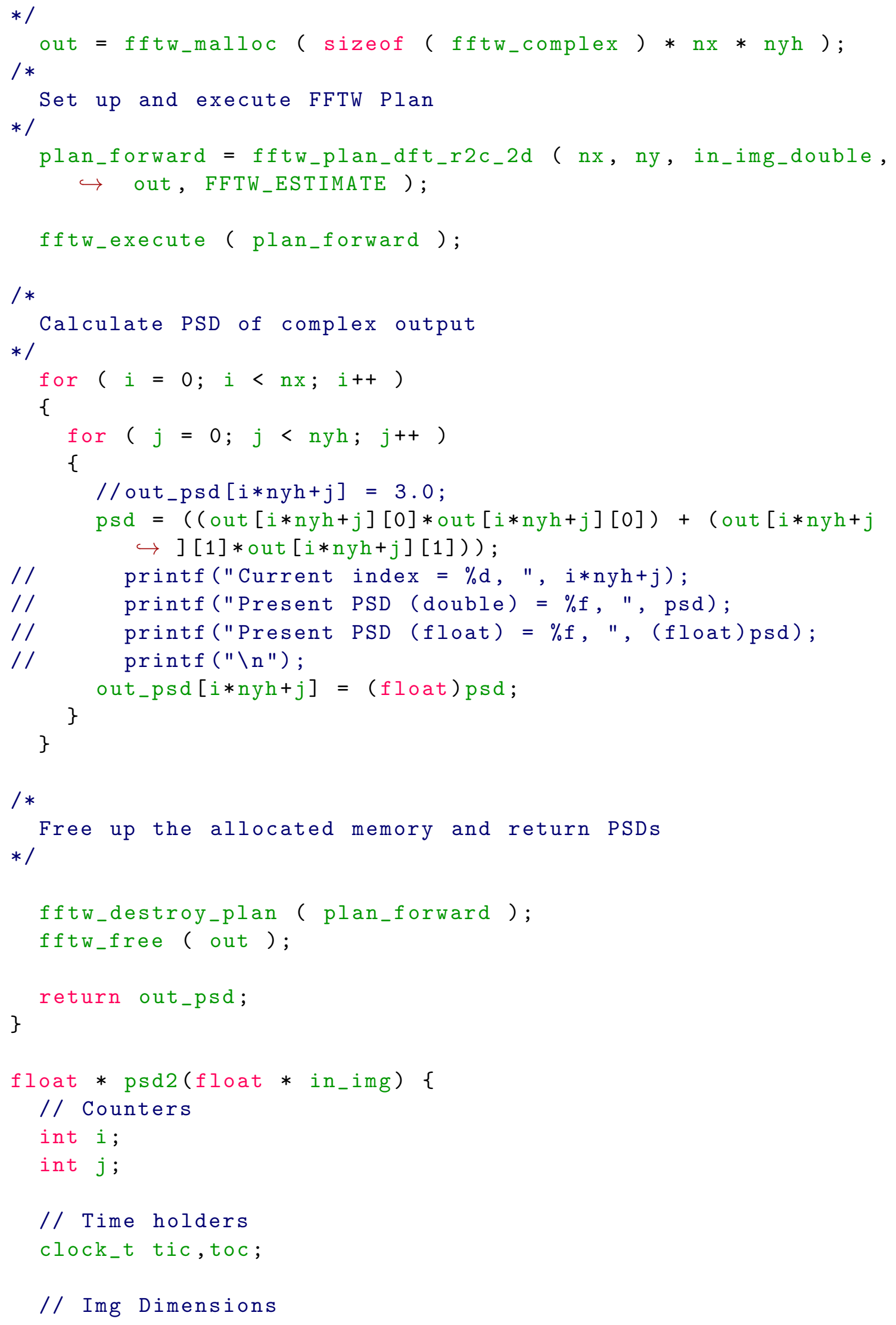




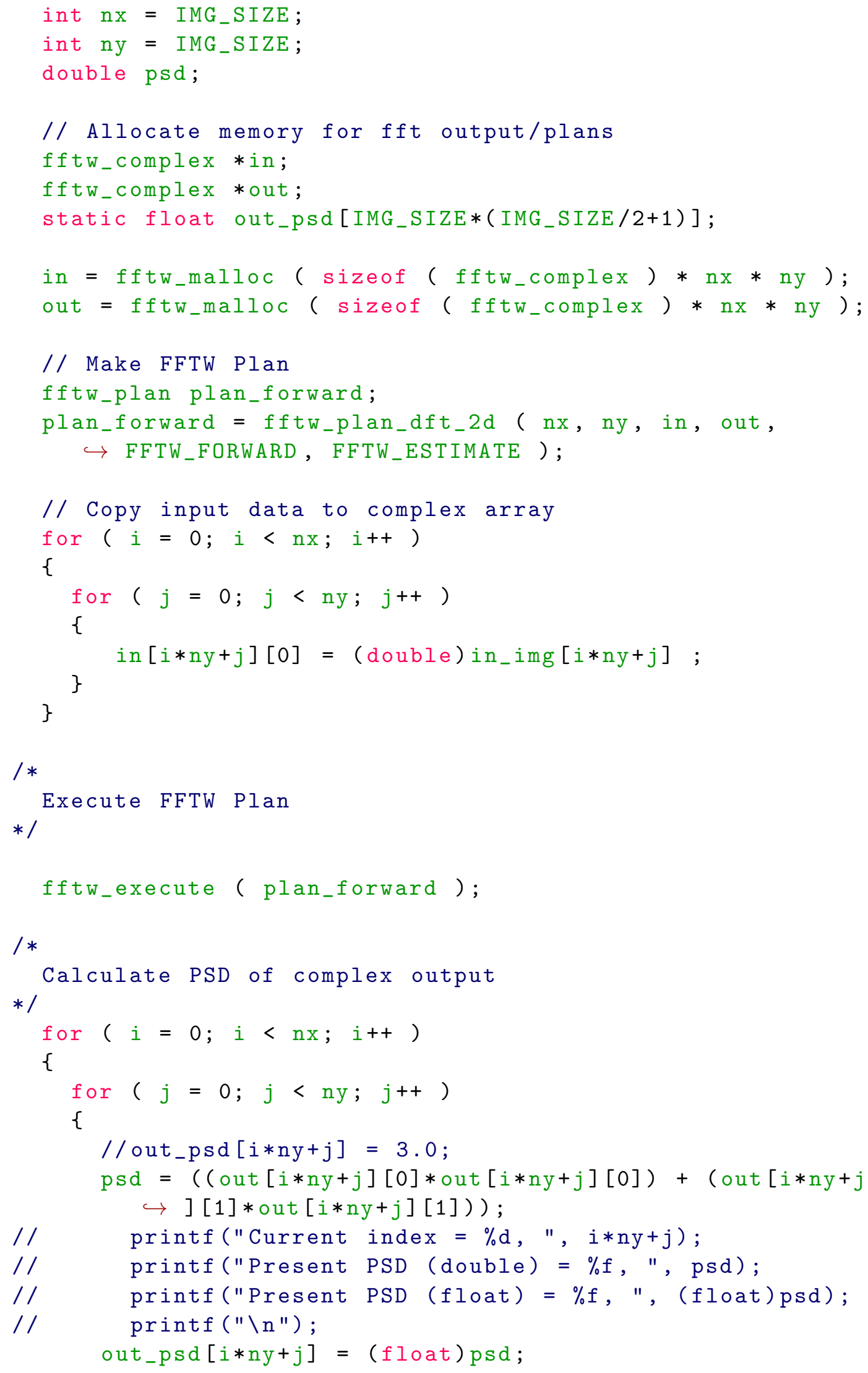




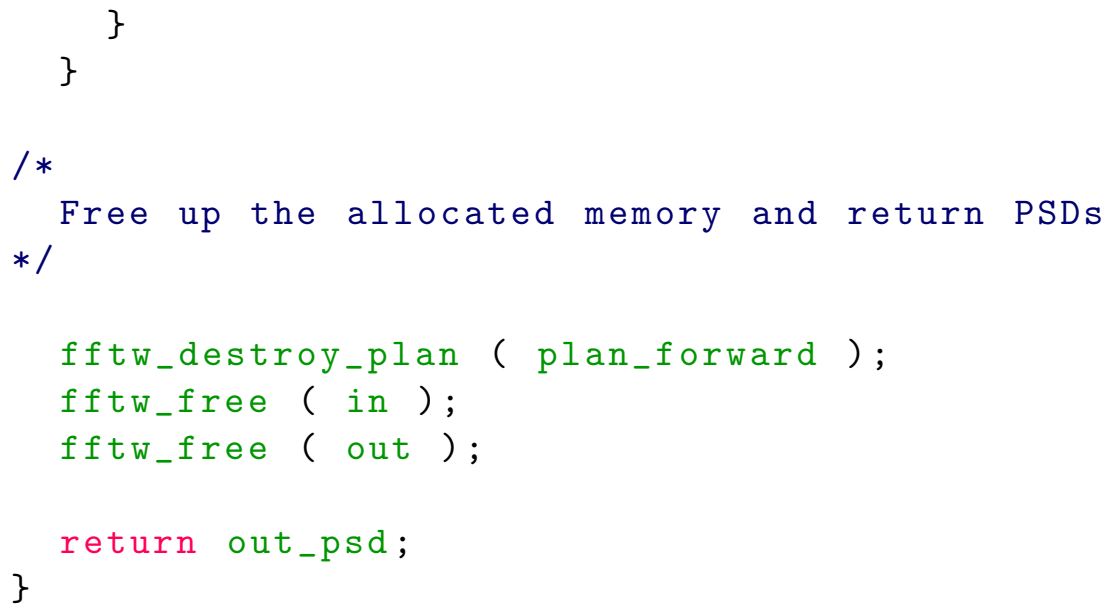

